\title{
COMPUTATIONAL STUDY OF INFRARED SPECTRA OF SILICA POLYMORPHS VIA CLASSICAL MECHANICS
}

\author{
A Thesis \\ presented to \\ the Faculty of the Graduate School \\ at the University of Missouri \\ In Partial Fulfillment \\ of the Requirements for the Degree \\ Master of Science \\ by \\ JIASEN GUO \\ Dr. Karl Hammond, Thesis Supervisor \\ DECEMBER 2017
}


(C) Copyright by Jiasen Guo 2017

All Rights Reserved 
The undersigned, appointed by the dean of the Graduate School, have examined the thesis entitled

\section{COMPUTATIONAL STUDY OF INFRARED SPECTRA OF SILICA POLYMORPHS VIA CLASSICAL MECHANICS}

presented by Jiasen Guo,

a candidate for the degree of Master of Science, and hereby certify that, in their opinion, it is worthy of acceptance.

Professor Karl Hammond, chair

Professor David Retzloff, member

Professor Heather Hunt, member

Professor Thomas Sewell, member 


\section{ACKNOWLEDGMENTS}

I would first like to thank my thesis advisor Professor Karl Hammond for his consistent and patient support on both my research work and thesis writing. His endless passion about computational modeling and programming have really enlightened me about the things that I am really interested in.

I would also like to thank the experts who advised me during this project,

- Professor David Retzloff of the Department of Chemical Engineering,

- Professor Heather Hunt of the Department of Bioengineering, and

- Professor Thomas Sewell of the Department of Chemistry,

for enlightening discussions and constructive suggestions on thesis writing.

Finally, I would like to express my gratitude to my friends Amir Mehdi Mofrad and A. Rafi Iasir for their kind help on zeolite knowledge and programming skills, respectively. 


\section{TABLE OF CONTENTS}

ACKNOWLEDGMENTS $\ldots \ldots \ldots \ldots \ldots \ldots \ldots \ldots \ldots$ ii

LIST OF FIGURES $\ldots \ldots \ldots \ldots \ldots \ldots \ldots \ldots \ldots \ldots \ldots \ldots \ldots \ldots$

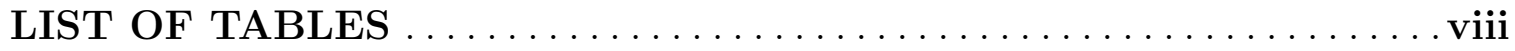

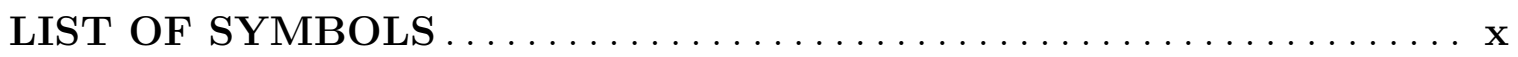

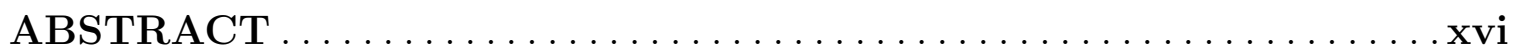

\section{Chapter}

1. INTRODUCTION $\ldots \ldots \ldots \ldots \ldots \ldots \ldots \ldots \ldots \ldots \ldots \ldots \ldots \ldots \ldots \ldots$

2. THEORETICAL BACKGROUND $\ldots \ldots \ldots \ldots \ldots \ldots \ldots \ldots \ldots$

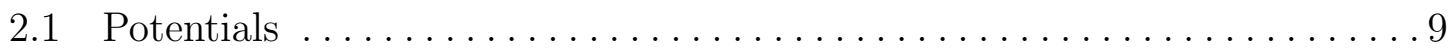

2.1.1 Potential Classification ............................. 11

2.1.2 Valence Potential. ............................... 19

2.1.3 Non-valence Potential ............................ 24

2.1.4 Potential Parameterization ......................... 34

2.2 Simulation Methods of Infrared Spectra $\ldots \ldots \ldots \ldots \ldots \ldots \ldots$

2.2 .1 Normal Mode Analysis . . . . . . . . . . . . . . . . . . . 38

$2.2 .2 \quad$ Molecular Dynamics ............................... 44

3. EVALUATION OF PUBLISHED POTENTIALS $\ldots \ldots \ldots \ldots \ldots 52$

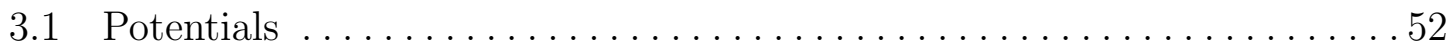

3.1.1 The MZHB Potential . . . . . . . . . . . . . . . . . . . . . . . 52

3.1 .2 The Core-Shell Model .............................. 55

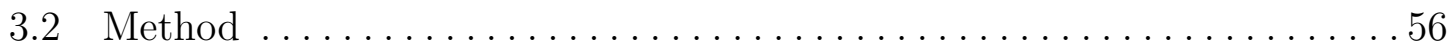

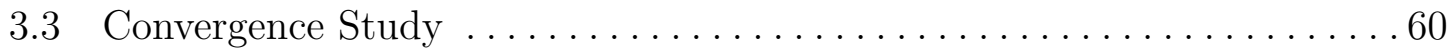


3.3.1 Effects of System Size ............................. 62

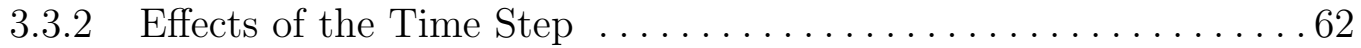

3.3.3 Effects of Sampling Frequency $\ldots \ldots \ldots \ldots \ldots \ldots \ldots \ldots \ldots \ldots$

3.3.4 Effects of the Initial Atomic Velocities and Simulation Length.................................66

3.3.5 Effects of the shell/core mass ratio .................. 70

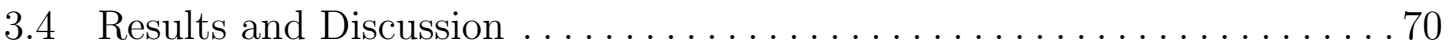

3.4.1 Geometric Observables and Bulk Moduli at $300 \mathrm{~K} \ldots \ldots \ldots \ldots 70$

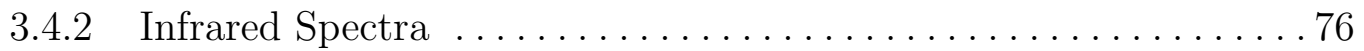

3.5 The Lennard-Jones Parameters of the MZHB Potential . . . . . . . . . . . 96

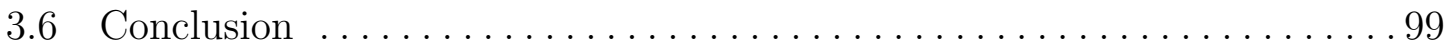

4. NEW POTENTIAL . . . . . . . . . . . . . . . . . . . . . . . 101

4.1 Sensitivity Analysis of the MZHB Potential .................... 102

4.2 The New Potential..................................... 105

4.3 Results and Discussion ................................ 107

4.3.1 Geometric Observables and Bulk Moduli at $300 \mathrm{~K} \ldots \ldots \ldots$. . . 107

4.3 .2 Infrared Spectra ............................... 108

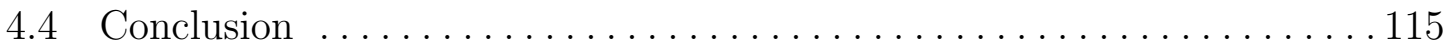

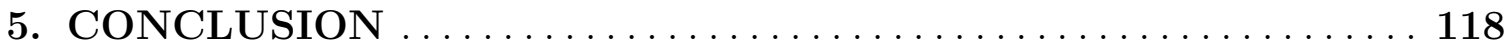

\section{Appendix}

\section{A. MD-PREDICTED UNIQUE ELASTIC TENSOR}

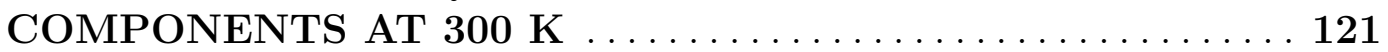

B. IR SPECTRA OF QUANTUM MECHANICAL CLUSTERS . . . . 124

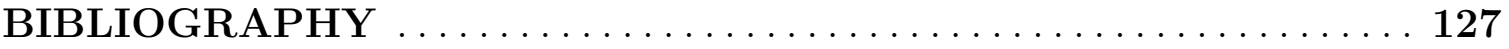




\section{LIST OF FIGURES}

$\begin{array}{lll}\text { Figure } & \text { Page }\end{array}$

1.1 Zeolites in industry and the natural world $\ldots \ldots \ldots \ldots \ldots \ldots \ldots \ldots$

1.2 Zeolite structures from a microscopic perspective $\ldots \ldots \ldots \ldots \ldots \ldots$

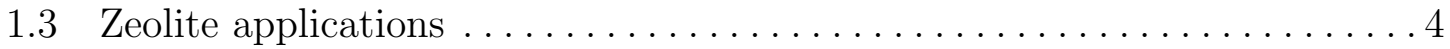

1.4 An illustration of water adsorption in zeolites via two different

mechanisms . . . . . . . . . . . . . . . . .

1.5 An illustration of the application of IR spectroscopy in the

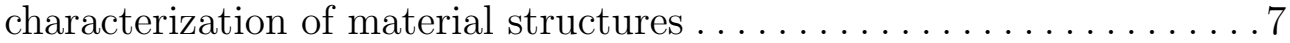

2.1 Illustrations of potential terms for an ethane molecule $\ldots \ldots \ldots \ldots \ldots 11$

2.2 Illustrations of improper potential and non-valence interactions . . . . . . 12

2.3 An illustration of the $1-4$ scale method $\ldots \ldots \ldots \ldots \ldots \ldots \ldots \ldots \ldots$

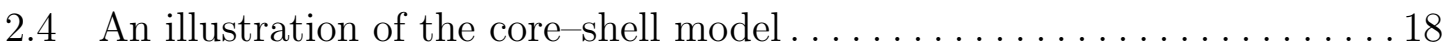

2.5 A graphical comparison of the harmonic bond potential and the Morse potential ..........................20

2.6 Illustrations of charge-dipole and dipole-dipole interactions $\ldots \ldots \ldots 27$

2.7 Illustrations of the van der Waals interactions $\ldots \ldots \ldots \ldots \ldots \ldots \ldots \ldots$

2.8 An illustration of the Lennard-Jones potential . . . . . . . . . . . . . . . 35

3.1 Illustrations of the cluster model used for peak assignments and the $4 \mathrm{R}$ vibrational analysis . . . . . . . . . . . . . . . . . 60

3.2 MZHB-predicted IR spectra for quartz with different system sizes . . . . 63

3.3 The total energy evolution during FAU simulations with different

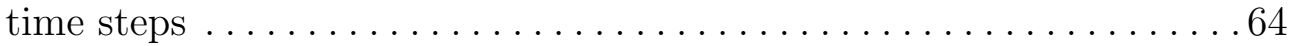


3.4 FAU IR spectra calculated with three different time steps $\ldots \ldots \ldots \ldots 6$

3.5 An illustration of aliasing in sample acquisition $\ldots \ldots \ldots \ldots \ldots \ldots \ldots$

3.6 FAU IR spectra dependence on the choice of sampling frequency . . . . 68

3.7 FAU IR spectra dependence on the random number seed . . . . . . . 70

3.8 FAU IR spectra dependence on the simulation length $\ldots \ldots \ldots \ldots \ldots 71$

3.9 The evolution of the total energy of three simulations with varying

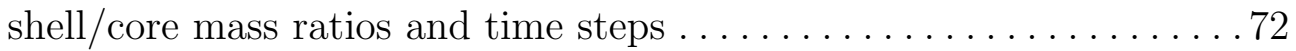

3.10 SOD bond length and angle distributions at $300 \mathrm{~K} \ldots \ldots \ldots \ldots 75$

3.11 SOD radial distribution functions at $300 \mathrm{~K} \ldots \ldots \ldots \ldots \ldots \ldots \ldots$

3.12 IR spectra of the cluster model from the cluster calculation $\ldots \ldots \ldots$. . 80

3.13 FAU IR spectra predicted by the MZHB potential at $300 \mathrm{~K} \ldots \ldots$. . . . 81

3.14 Visualizations of the FAU IR mode at $453 \mathrm{~cm}^{-1}$ predicted by the MZHB potential and the cluster IR mode at $414 \mathrm{~cm}^{-1}$ predicted

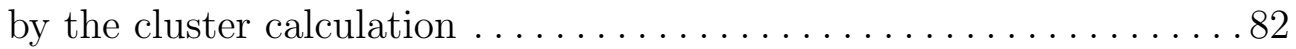

3.15 An illustration of the motions of oxygen atoms in an attached $6 \mathrm{R}$ associated with the $4 \mathrm{R}$ breathing motion .............. 83

3.16 Visualizations of the FAU IR mode at $564 \mathrm{~cm}^{-1}$ predicted by the MZHB potential and the cluster IR mode at $460 \mathrm{~cm}^{-1}$ predicted by the cluster calculation $\ldots \ldots \ldots \ldots \ldots \ldots \ldots \ldots \ldots \ldots \ldots \ldots \ldots \ldots$

3.17 Visualizations of the FAU IR mode at $528 \mathrm{~cm}^{-1}$ predicted by the MZHB potential and the cluster IR mode at $446 \mathrm{~cm}^{-1}$ predicted

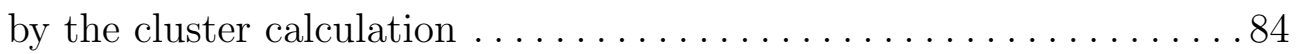

3.18 The 4-membered ring vibrational frequency analysis . . . . . . . . . . 85

3.19 FAU IR spectra predicted by the core-shell model at $300 \mathrm{~K} \ldots \ldots$. . . . 86

3.20 Visualization of the FAU IR peak at around $370 \mathrm{~cm}^{-1}$ predicted by

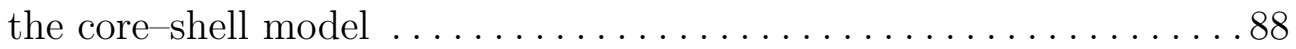

3.21 SOD IR spectra predicted by both the MZHB potential and the core-shell model at $300 \mathrm{~K}$ 
3.22 Visualization of the SOD peak at around $740 \mathrm{~cm}^{-1}$ predicted by the MZHB potential ..............................

3.23 Quartz IR spectra predicted by both the MZHB potential and the

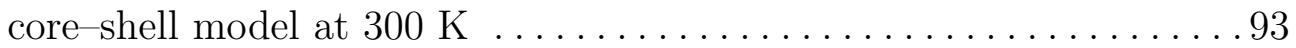

3.24 Cristobalite IR spectra predicted by both the MZHB potential and

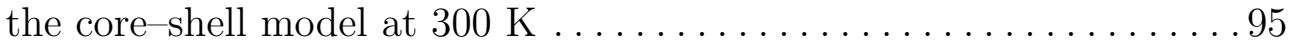

3.25 MFI IR spectra predicted by both the MZHB potential and the

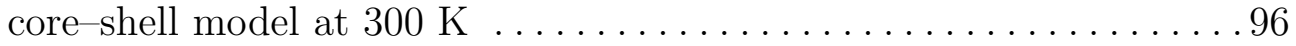

3.26 A comparison of the Lennard-Jones potential using parameters from CHARMM and from MZHB ........................ 99

4.1 Sensitivity analysis of the siliceous zeolite lattice parameter dependence on model parameters at $0 \mathrm{~K}$................... 104

4.2 Sensitivity analysis of the siliceous zeolite bulk modulus dependence

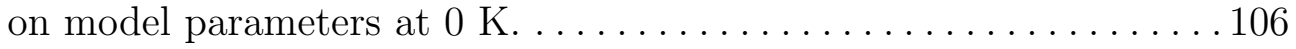

4.3 A comparison of FAU IR spectra predicted by the MZHB potential and the new potential from MD simulations at $300 \mathrm{~K} \ldots \ldots \ldots \ldots 110$

4.4 A comparison of SOD IR spectra predicted by the MZHB potential and the new potential from MD simulations at $300 \mathrm{~K} \ldots \ldots \ldots . .112$

4.5 A comparison of quartz IR spectra predicted by the MZHB potential and the new potential from MD simulations at $300 \mathrm{~K} \ldots \ldots \ldots . .113$

4.6 A comparison of cristobalite IR spectra predicted by the MZHB potential and the new potential from MD simulations at

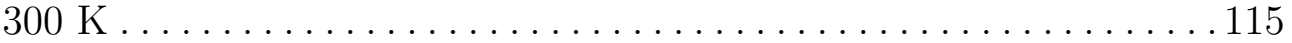

4.7 A comparison of MFI IR spectra predicted by the MZHB potential and the new potential from MD simulations at $300 \mathrm{~K} \ldots \ldots . . . .116$

B.1 Cluster models used in quantum mechanical calculations ........... 125

B.2 IR spectra of clusters used in this work $\ldots \ldots \ldots \ldots \ldots \ldots \ldots \ldots \ldots \ldots$ 


\section{LIST OF TABLES}

Table

Page

2.1 Typical components of zeolite potentials categorized by the number

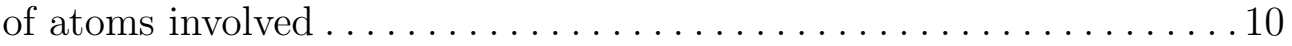

2.2 Typical components of potentials categorized by atomic bonding

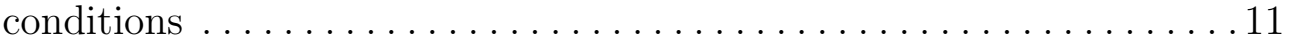

2.3 Typical components of a CHARMM-style potential. . . . . . . . . . 16

2.4 Typical cross interaction terms used in valence potentials . . . . . . . . . 24

2.5 Example of partial charges for Si atoms used in classical MD

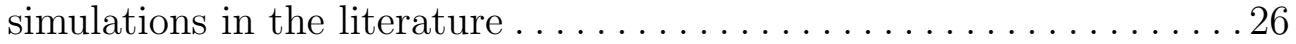

2.6 Basic simulation parameters in MD simulations $\ldots \ldots \ldots \ldots \ldots \ldots \ldots$

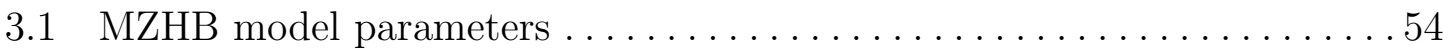

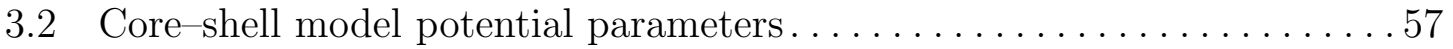

3.3 MD simulation sizes and parameters adopted in this work . . . . . . 61

3.4 Silica polymorphs' geometric observables and bulk moduli predicted by the MZHB potential and the core-shell model . . . . . . . . . 73

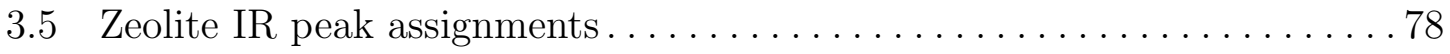

3.6 A comparison of the calculated and the measured FAU IR

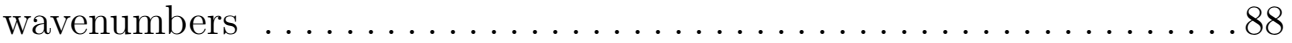

3.7 A comparison of the MD-calculated and the measured SOD IR

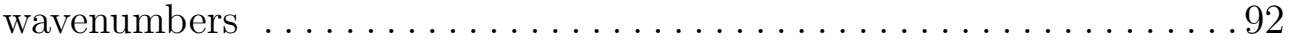

3.8 A comparison of the MD-calculated and the measured quartz IR wavenumbers 
3.9 A comparison of MD-calculated and measured cristobalite IR wavenumbers .................................. 94

3.10 The Lennard-Jones parameters used in the MZHB potential ..........97

3.11 The Lennard-Jones parameters published in the work of Lopes et al. ................................ 97

4.1 Model parameters of the new potential . . . . . . . . . . . . . . 107

4.2 Model-predicted silica polymorphs' average geometric observables and bulk moduli............................. 109

4.3 Peak assignments of FAU IR peaks predicted by the MZHB potential and the new potential...................... 111

4.4 Peak assignments of SOD IR peaks predicted by the MZHB potential and the new potential....................... 111

4.5 Peak assignments of quartz IR peaks predicted by the MZHB potential and the new potential...................... 113

4.6 Peak assignments of cristobalite IR peaks predicted by the MZHB potential and the new potential....................... 114

A.1 MD-predicted FAU stiffness tensor components, bulk modulus $K$ and shear modulus $G$ at $300 \mathrm{~K}$, in $\mathrm{GPa} \ldots \ldots \ldots \ldots \ldots \ldots \ldots . \ldots . \ldots . \ldots 121$

A.2 MD-predicted SOD elastic tensor components, bulk modulus $K$, and

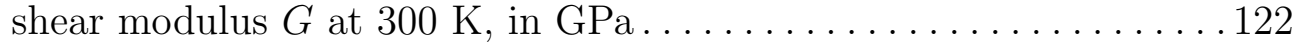

A.3 MD-predicted quartz elastic tensor components, bulk modulus $K$, and shear modulus $G$ at $300 \mathrm{~K}$, in GPa ................. 122

A.4 MD-predicted cristobalite elastic tensor components, bulk modulus $K$, and shear modulus $G$ at $300 \mathrm{~K}$, in GPa ............... 122

A.5 MD-predicted MFI elastic tensor components, bulk modulus $K$, and shear modulus $G$ at $300 \mathrm{~K}$ in $\mathrm{GPa} \ldots \ldots \ldots \ldots \ldots \ldots \ldots \ldots \ldots . \ldots \ldots$ 


\section{LIST OF SYMBOLS}

A Constant in the Buckingham potential, page 10

$A(t) \quad$ Instantaneous value of the studied quantity of the simulated system, see equation (2.56), page 49

$A_{i} \quad$ Amplitude of atomic displacement of atom $i$, see equation (2.41), page 41

C Constant in the Buckingham potential, page 10

$C_{\text {dispersion }} \quad$ Coefficient of London forces, see equation (2.26), page 33

$C_{\text {induction }} \quad$ Coefficient of Debye forces, see equation (2.26), page 33

$C_{\text {orientation }}$ Coefficient of Keasom forces, see equation (2.26), page 33

$C_{i j} \quad$ Material elastic constant, see equation (4.1), page 102

$D_{e} \quad$ Bond dissociation energy, page 10

$D_{i} \quad$ Drude particle for atom $i$, see equation (2.23), page 29

$D_{k, i} \quad$ Element of the eigenvector matrix, see equation (2.46), page 43

E Total energy of the simulated system, see equation (2.56), page 49

$H_{i j} \quad$ Hessian matrix elements, see equation (2.37), page 40

$I(\nu) \quad$ Intensity of the infrared peak of frequency $\nu$, see equation (2.62), page 51

$I_{k} \quad$ Infrared intensity of the $k^{t h}$ normal mode, see equation (2.44), page 42

$J_{i i, 0} \quad$ Atomic hardness of atom $i$, see equation (2.19), page 28

$J_{i j} \quad$ Coulomb interaction energy between unit charges, see equation (2.19), page 28

$K \quad$ Bulk modulus, see equation (4.1), page 102

$L \quad$ Lagrangian, see equation (2.39), page 41

$M_{j} \quad$ Cartesian components of the molecular dipole moment, see equation (2.44), page 42

$N \quad$ Total number of atoms, see equation (2.19), page 28 
$N_{A} \quad$ Avogadro's number, see equation (2.52), page 44

$Q_{k} \quad k^{t h}$ element of the normal coordinate vector, see equation (2.44), page 42

$R_{0}, R \quad$ Equilibrium and instantaneous bond lengths, page 10

$R_{i j} \quad$ Instantaneous bond length between atoms $i$ and $j$, see equation (2.8), page 22

$S \quad$ A quantity proportional to the area of a four-membered ring, see equation (3.13), page 60

$T(t) \quad$ The instantaneous temperature of the simulated system in molecular dynamics simulations, see equation (2.54), page 48

$T^{*} \quad$ The desired temperature (set point) in a molecular dynamics simulation, see equation (2.54), page 48

$U_{i}^{\mathrm{QM}} \quad$ Electrostatic potential at the position of atom $i$ calculated from quantum mechanics, see equation (2.30), page 36

$U_{\mathrm{EL}, i} \quad$ Electrostatic potential at the position of atom $i$, see equation (2.17), page 27

$V \quad$ Total potential energy, page 10

$V_{0} \quad$ Total potential energy of the local minimum-energy configuration, see equation (2.1), page 13

$V_{\mathrm{EL}} \quad$ Electrostatic potential energy component, see equation (2.5), page 16

$V_{\mathrm{UB}} \quad$ Urey-Bradley potential energy component, see equation (2.5), page 16

$V_{\text {angle }} \quad$ Bond angle potential energy component, see equation (2.4), page 14

$V_{\text {bond }} \quad$ Bond length potential energy component, see equation (2.4), page 14

$V_{c-c} \quad$ Charge-charge interaction energy component of the electrostatic interaction energy, see equation (2.13), page 26

$V_{\mathrm{c}-\mathrm{d}} \quad$ Charge-dipole interaction energy component of the electrostatic interaction energy, see equation (2.13), page 26

$V_{\text {core-shell }} \quad$ Self-polarization potential energy component in the core-shell model, see equation (2.6), page 18

$V_{\mathrm{d}-\mathrm{d}} \quad$ Dipole-dipole interaction energy component of the electrostatic interaction energy, see equation (2.13), page 26

$V_{\text {dihedral }} \quad$ Dihedral potential energy component, see equation (2.5), page 16 
$V_{\text {else }} \quad$ Other potential energy components that are not related to the separation distance between the physical-Drude particle pair in the Drude model, see equation $(2.24)$, page 30

$V_{\text {improper }} \quad$ Improper potential energy component, see equation (2.0), page 10

$V_{\text {polar }} \quad$ Polarization energy required to polarize the atoms in the point dipole method, see equation (2.16), page 27

$V_{\text {short-range }}$ Short-range potential energy component, see equation (2.6), page 18

$V_{\text {sys }}^{\text {induced }} \quad$ The energy of an induced dipole system, see equation (2.16), page 27

$V_{\mathrm{vdW}} \quad$ van der Waals potential energy component, see equation (2.5), page 16

$V_{n} \quad$ Dihedral potential energy barrier, page 10

$V_{i, 0} \quad$ Potential energy of the charge neutral atom $i$, see equation (2.19), page 28

$V_{i j} \quad$ Pair-wise potential energy between atom $i$ and $j$, see equation (2.11), page 24

$Z_{i} \quad$ Atomic number of atom $i$, see equation (2.14), page 26

$\Delta t \quad$ Time step used in the numerical integration of Newton's equations of motion, see equation (3.14), page 65

$\Delta t_{s} \quad$ Sampling interval between in two data points in time unit, see equation (3.14), page 65

$\Gamma \quad$ One single point in the phase space, see equation (2.57), page 49

$\Phi_{0}, \Phi \quad$ Equilibrium and instantaneous out-of-plane angles in improper potentials, see equation (2.0), page 10

$\alpha \quad$ Atomic polarizability, see equation (2.8), page 22

$\chi_{i} \quad$ Electronegativity of atom $i$, see equation (2.19), page 28

$\delta_{i j} \quad$ Kronecker delta, see equation (2.42), page 41

$\epsilon_{0} \quad$ Vacuum permittivity, see equation (2.11), page 24

$\epsilon_{i} \quad$ Energy well depth for the interaction of two atoms of type $i$, see equation (2.31), page 38

$\epsilon \quad$ Energy well depth in the Lennard-Jones interactions, page 10

$\gamma \quad$ Eigenvalue of the Hessian matrix, see equation (2.41), page 41

$\lambda \quad$ Charge parameter in the point dipole method, see equation (2.17), page 27 
$\langle A\rangle \quad$ Ensemble average of the quantity $A$, see equation (2.56), page 49

D Eigenvector matrix, see equation (2.45), page 42

$\mathcal{H} \quad$ The Hamiltonian, see equation (2.55), page 48

$\mathcal{H}_{0} \quad$ Hamiltonian of the unperturbed system, see equation (2.57), page 49

$\mathcal{Q} \quad$ "Mass" of the additional degree of freedom $s$ in the Nosé-Hoover thermostat, see equation (2.55), page 48

$\mu_{i} \quad$ Chemical potential of atom $i$, see equation (2.20), page 29

$\nu_{s} \quad$ Sampling frequency, see equation (3.14), page 65

$\nu \quad$ Frequency of infrared peaks, see equation (2.62), page 51

$\nu_{i} \quad$ Vibrational frequency of the $i^{\text {th }}$ normal mode, see equation (2.43), page 42

$\phi_{0}, \phi \quad$ Equilibrium and instantaneous phase angles of the dihedral, page 10

$\rho \quad$ Distance parameter in the Born-Mayer potential, see equation (3.8), page 55

$\theta_{i j k, 0}, \theta_{i j k} \quad$ Equilibrium and instantaneous angles formed by atoms $i, j$ and $k$, with atom $j$ being the center atom, page 10

$\varphi \quad$ Phase angle, see equation (2.41), page 41

$\mathbf{E}_{\mathbf{i}} \quad$ Electric field at the position of atom $i$, see equation (2.27), page 33

M Molecular dipole moment, see equation (2.44), page 42

Q Normal coordinate vector, see equation (2.45), page 42

$\boldsymbol{\mu}_{\mathbf{j}} \quad$ Dipole moment at position of atom $j$, see equation (2.14), page 26

d Vector of separation distances between all the physical-Drude particle pairs, see equation (2.24), page 30

$\mathbf{p}_{\mathbf{i}} \quad$ Momentum of the coordinates of atom $i$, see equation (2.55), page 48

$\mathbf{p}_{\mathbf{s}} \quad$ Momentum of the additional degree of freedom $s$ in the Nosé-Hoover thermostat, see equation (2.55), page 48

q Mass-weighted coordinate vector, see equation (2.45), page 42

$\mathbf{r}_{\mathbf{i j}} \quad$ Displacement vector from atom $j$ to $i$, see equation (2.14), page 26

$\mathbf{r} \quad$ Vector of the positions of all the physical particles in the Drude model, see equation (2.24), page 30 
$\zeta \quad$ External perturbation in linear response theory, see equation (2.57), page 49

a Bond strength constant in Morse potential, page 10

c Speed of light in vacuum, see equation (2.52), page 44

e Elementary charge, see equation (2.14), page 26

$f_{i j} \quad$ Pair-wise force between atoms $i$ and $j$, see equation (2.11), page 24

$g\left(q_{1}, q_{2} \ldots\right)$ Charge conservation constraint in potential map method for atomic charge determination, see equation (2.30), page 36

$g(r) \quad$ Radial distribution function, see equation (3.17), page 76

$h \quad$ Number of degrees of freedom of the physical system in the Nosé-Hoover thermostat, see equation (2.55), page 48

$k \quad$ Dielectric constant, see equation (2.12), page 25

$k_{B} \quad$ Boltzmann's constant, see equation (2.55), page 48

$k_{R} \quad$ Harmonic bond potential force constant, page 10

$k_{\Phi} \quad$ Improper potential force constant, see equation (2.0), page 10

$k_{\theta} \quad$ Harmonic angle potential force constant, page 10

$k_{R, \phi} \quad$ Bond--dihedral cross interaction force constant, see equation (2.2), page 13

$k_{R, \theta} \quad$ Bond-angle cross interaction force constant, see equation (2.2), page 13

$k_{R, i} \quad$ Force constant for higher-order energy correction in bond potential, see equation (2.4), page 14

$k_{U B} \quad$ Urey-Bradley potential force constant, page 10

$k_{\theta_{1}, \theta_{2}} \quad$ Angle-angle cross interaction force constant, page 10

$k_{\theta, \phi} \quad$ Angle-dihedral cross interaction force constant, see equation (2.2), page 13

$k_{\theta, i} \quad$ Force constant for higher-order energy correction in angle potential, see equation (2.4), page 14

$m_{i} \quad$ Mass of atom $i$, see equation (2.33), page 40

$n \quad$ Integer representing the periodicity of the dihedral potential, page 10

$n(r) \quad$ Number of particles located inside the spherical shell at a distance $r$ from the center, see equation (3.17), page 76

$\Delta q_{i} \quad$ Increment of the coordinate $q_{i}$, see equation (2.35), page 40 


\begin{tabular}{|c|c|}
\hline$\ddot{q}_{i}$ & Generalized acceleration, see equation (2.40), page 41 \\
\hline$\dot{q}_{i}$ & Velocity, see equation (2.34), page 40 \\
\hline$i_{i}$ & Atomic charge of atom $i$, see equation (2.11), page 24 \\
\hline$i$ & $\begin{array}{l}\text { Mass-weighted coordinates of degree of freedom } i \text {, see equation (2.33), } \\
\text { page } 40\end{array}$ \\
\hline$q_{D_{i}}$ & Charge of Drude particle $D_{i}$, see equation $(2.23)$, page 29 \\
\hline $0, r$ & Equilibrium and instantaneous internuclear distances, page 10 \\
\hline$m$ & $\begin{array}{l}\text { Minimum-energy internuclear distance in the Lennard-Jones potential, } \\
\text { page } 10\end{array}$ \\
\hline$r_{i j}$ & $\begin{array}{l}\text { Instantaneous internuclear distance between two atoms } i \text { and } j \text {, see equa- } \\
\text { tion }(2.11) \text {, page } 24\end{array}$ \\
\hline$m, i$ & $\begin{array}{l}\text { Minimum-energy internuclear distance in the Lennard-Jones potential for } \\
\text { atoms of type } i \text {, see equation }(2.31) \text {, page } 38\end{array}$ \\
\hline$s$ & $\begin{array}{l}\text { The additional degree of freedom in the Nosé-Hoover thermostat, see } \\
\text { equation }(2.55) \text {, page } 48\end{array}$ \\
\hline$t$ & Time, see equation (2.32), page 39 \\
\hline$t_{0}$ & Time origin of time correlation functions, see equation (2.60), page 50 \\
\hline$t_{\text {tot }}$ & Total simulation length, see equation (2.56), page 49 \\
\hline$x_{i}, y_{i}, z_{i}$ & Cartesian coordinates of atom $i$, see equation (2.32), page 39 \\
\hline ( & $\begin{array}{l}\text { Objective function in potential map method for atomic charge determi- } \\
\text { nation, see equation }(2.30) \text {, page } 36\end{array}$ \\
\hline $4 \mathrm{R}$ & Four-membered ring consists of four Si atoms, page 60 \\
\hline $6 \mathrm{R}$ & Six-membered ring consists of six Si atoms, page 60 \\
\hline D6R & $\begin{array}{l}\text { Double six-membered ring cluster formed by two six-membered rings, } \\
\text { page } 60\end{array}$ \\
\hline
\end{tabular}




\title{
COMPUTATIONAL STUDY OF INFRARED SPECTRA OF SILICA POLYMORPHS VIA CLASSICAL MECHANICS
}

\author{
Jiasen Guo \\ Dr. Karl Hammond, Thesis Supervisor
}

\begin{abstract}
A potential energy model that correctly reflects zeolite framework interactions is the premise for computational studies of the physical and chemical processes occurring inside zeolites, such as catalytic chemical reactions and adsorption. Infrared spectroscopy is a widely-used technique that is sensitive to the accuracy of the potential energy model. This work aims to develop such a potential that reproduces the infrared spectra of zeolites. In the first part of this thesis, the performance of two published potentials is tested in terms of predicting structural and dynamical properties for five silica polymorphs (three siliceous zeolites: siliceous faujasite, sodalite and silicalite; quartz; and cristobalite). Comparison between the silica polymorphs' model-predicted equilibrium angle distributions and infrared spectra shows that the core-shell model [Schröder and Sauer, J. Phys. Chem. 1996, 100, 11043] predicts a broader $\mathrm{Si}-\mathrm{O}-\mathrm{Si}$ angle distribution and shifts angle-bending infrared modes to lower wavenumbers. The MZHB potential [Sahoo and Nair, J. Comput. Chem. 2015, 36, 1562], on the other hand, predicts angle-bending infrared modes that are consistently shifted to higher wavenumbers. The second part of this thesis presents a new potential via reparameterizing and extending the MZHB potential based on a sensitivity analysis, which investigates the relationships between model parameters and the structural properties of silica polymorphs. Better infrared predictions are achieved by the new potential. The results of the sensitivity analysis indicate that the lattice parameter might be a possible target for the parameterization of atomic partial charges for crystalline materials.
\end{abstract}




\section{CHAPTER 1}

\section{INTRODUCTION}

Zeolites are a type of crystalline material consisting mainly of the elements silicon, oxygen, and aluminum. Unlike those used in industry, zeolites in the natural world have various colors that depend on the types of impurities, as shown in Figure 1.1. While a relatively small portion of zeolitic materials contain only silicon and oxygen elements, called siliceous zeolites ${ }^{1}$ the majority of zeolites contain aluminum and extra-framework cations, and are often called aluminosilicates. In an aluminosilicate, some of the zeolite framework silicon atoms are replaced by aluminum atoms. Due to the different bonding properties of silicon and aluminum - that is, silicon atoms have four valence electrons, while aluminum atoms have only three valence electronsreplacement of silicon atoms with aluminum atoms introduces net negative charges into the zeolite structure. As such, hydrogen ions $\left(\mathrm{H}^{+}\right)$or other charge-compensating cations must be present when aluminum is present in the framework.

Different frameworks of zeolites are given three-letter codes. Some of the codes are taken from the first three capitalized letters in the name of the corresponding zeolites, such as sodalite (SOD) and faujasite $(\mathbf{F A U})$; some other codes have more specific meanings. For example, the code MFI originates from ZSM-5, which is a catalyst patented by Mobil oil company (M-Mobil, FI-5). A full list of codes for zeolites that are currently accepted is available on the International Zeolite Association (IZA) [1].

\footnotetext{
${ }^{1}$ Technically speaking, compounds with only silicon and oxygen element are called zeolite-like compounds. In this thesis, we use the term "siliceous zeolites" to emphasize the relationships between zeolites and these compounds.
} 


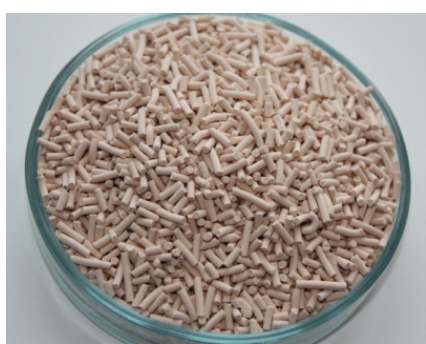

(a)

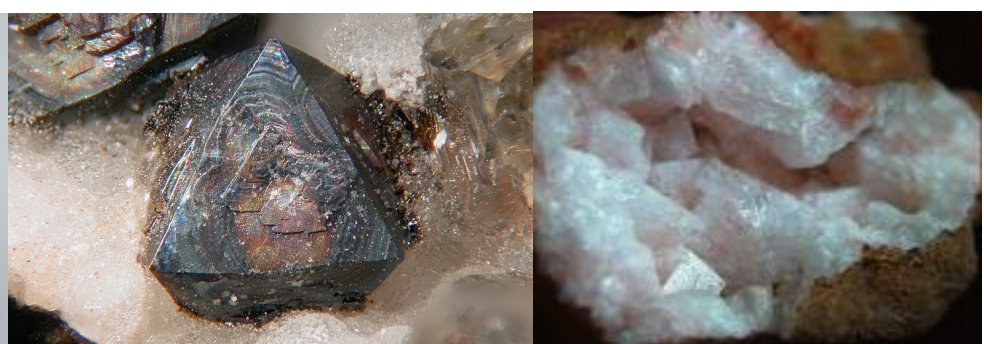

(b)

(c)

Figure 1.1. Zeolites in industry and the natural world: (a) extruded NaX synthetic zeolite (FAU framework) [2]; (b) the natural zeolite Ca-faujasite (FAU), coated with black oxides [3]; (c) the natural zeolite chabazite (CHA) [4]. While zeolites in the natural world often grow as large crystals, synthetic zeolites adopted in industry typically consist of very small crystals; the resulting powders are often extruded or pressed into pellets to make them easier to handle.

Zeolites are also microporous materials with pores and channels of different sizes, as illustrated in Figure 1.2. These pore structures are formed by rings characterized by different numbers of $\mathrm{SiO}_{4}$ tetrahedrons, as shown in Figure 1.2c. The presence of aluminum and the resulting acid sites, together with the microporous nature of zeolites, leads to the wide application of zeolites in the petrochemical industry as acid catalysts to enhance petrochemical reactions. Some of the most important properties are:

Lewis or Brønsted acidity originates from $\mathrm{H}^{+}$(Brønsted) and/or aluminum-based framework charge (Lewis)

Shape selection originates from the relative sizes of reacting species and zeolite pores

Ion-exchange originates from the energy-change upon replacements between ions

Acidity in zeolites originates from charge-compensating $\mathrm{H}^{+}$and cations. In the case of $\mathrm{H}^{+}$, the zeolite serves as a proton donor [7]. For example, zeolite H-ZSM-5 is widely used for the conversion of methanol to dimethyl ether via dehydration [8]. H-ZSM-5 


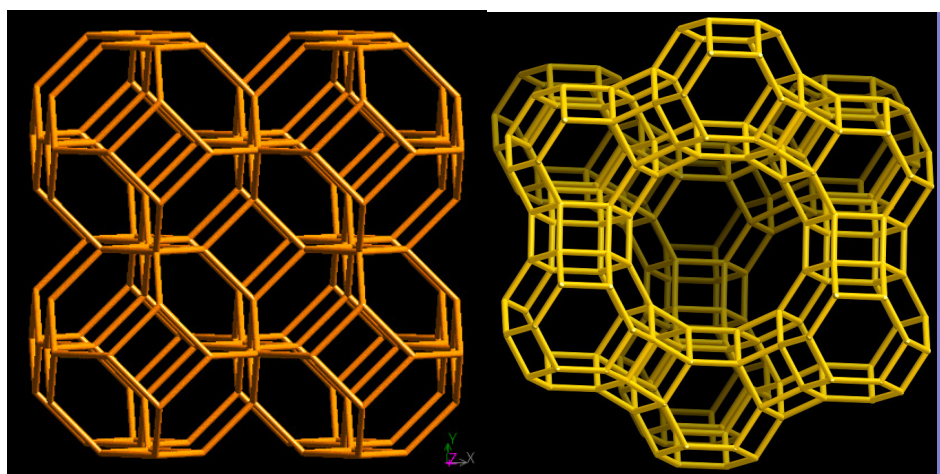

(a) The zeolite SOD (b) The zeolite FAU

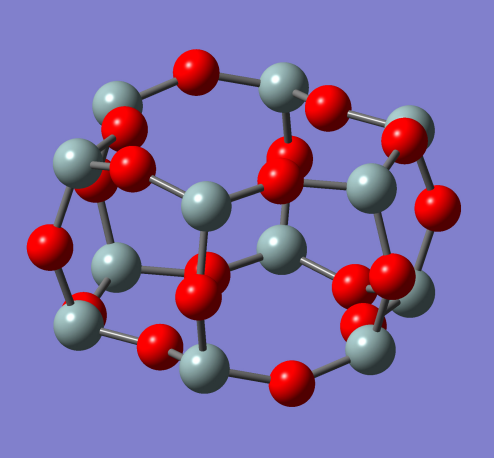

(c) A D6R of zeolite FAU

Figure 1.2. Zeolite structures from a microscopic perspective: (a) a wire frame of the zeolite sodalite (SOD) [5]; (b) a wire frame of the zeolite faujasite (FAU) [6]; (c) a double 6-membered ring (D6R) of the zeolite faujasite: the D6R serves as a connecting part between sodalite cages in the FAU structure.

is also used in Fischer-Tropsch reactions to convert synthesis gas to hydrocarbons. In the case of other charge-compensating cations, e.g. $\mathrm{Na}^{+}, \mathrm{Ni}^{2+}$ or $\mathrm{Zn}^{2+}$, the zeolite serves as an electron acceptor during chemical reactions including dehydrogenation, carbonylation, and the catalytic cracking [9].

Another property of zeolites originates from zeolites' porous structure. Pores and channels of different sizes allow for shape-selective reactions in which only reactants and desired products can travel through the channels, such that reaction selectivity can be enhanced. A typical example of the utilization of this property is the production of $p$-xylene via toluene reacted with methanol with H-ZSM-5 zeolite illustrated in Figure 1.3b, where only $p$-xylene among the three isomers can diffuse through the zeolite channels [10]. Thus, it is clear that zeolite structures strongly affect the diffusivity of chemical species.

The ion-exchange property arises from the energy difference between varies ionzeolite complexes [11]. This property has led to the highest-volume use of zeolites in 


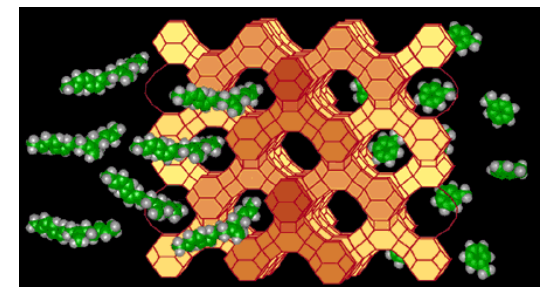

(a) Hydrocarbons react in a zeolite catalyst

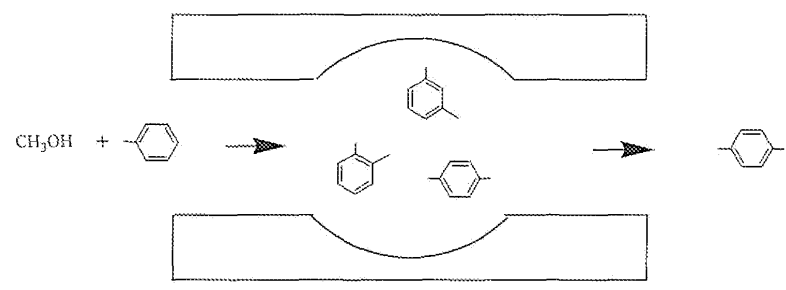

(b) Shape-selectivity of zeolite catalysts

Figure 1.3. Zeolite applications: (a) zeolite Y ( (FAU framework) is used as a hydrocarbon cracking catalyst in the petrochemical industry; the image is a caricature of hydrocarbons diffusing through zeolite channels during chemical reactions. Image from the International Zeolite Association [1]. (b) an illustration of zeolite shape-selectivity: in the reaction of toluene and methanol with H-ZSM-5 (MFI) as the catalysts, only $p$-xylene (and not $m$ xylene or $o$-xylene) is the right shape to diffuse through the zeolite channels, therefore, the reaction selectivity is enhanced. Image from Galloway et al. [13].

water treatment. For example, in water softening with zeolite resin, water hardness is often reduced via replacing $\mathrm{Ca}^{2+}$ and $\mathrm{Mg}^{2+}$ with $\mathrm{Na}^{+}$.

Other applications of zeolites include adsorption in water treatment, as organic pollutants such as phenol can be removed from water by natural zeolites at low cost [12]. Such adsorption processes are also used in the removal of small gas molecules, such as $\mathrm{CO}_{2}$ and $\mathrm{N}_{2}$. Therefore, a fundamental understanding of the dynamic and thermal properties of these processes is important in order to enhance the efficiency of these processes.

Instead of investigating these processes via direct experiments, computer modeling has been widely adopted to study dynamic and thermal properties of catalytic reactions and adsorption occurring inside zeolites, such as diffusion coefficients of reacting species and heats of adsorption. These computer simulations require a potential energy model describing the interactions between the zeolite framework (host), and any reacting species or adsorbate (guest). While fixed zeolite framework models have been widely adopted to model reactions and adsorption $[14,15]$, in which case the crystallographic structure of zeolite framework is not allowed to change upon interacting 
with guest molecules, it is more promising and natural to allow for the relaxation of the zeolite framework during simulations. The reason is that the zeolite framework is essentially vibrating all the time, and the electronic structure of the zeolite framework will also change because of the approach of a guest molecule. However, such a relaxed zeolite framework model will require that we have a good understanding about how zeolite framework atoms interact with each other, meaning that we need a good potential energy model (or "force field") to describe the zeolite framework interactions. A good potential for zeolite frameworks is also necessary when computational modeling of vibrational spectroscopy is used to detect whether certain reaction intermediates (known as transition states) are formed on the surface of the zeolite catalysts, or to examine different experimentally-observed adsorbed species. For example, it is well known that water molecules can be adsorbed in zeolites via two different mechanisms: molecular adsorption and dissociative adsorption [16]. The difference between these two adsorption mechanisms lies in whether the water molecule attaches to the zeolite framework as a whole molecule or a hydroxyl group with a dissociated proton, as is illustrated in Figure 1.4. The resulted different products can often be distinguished from each other via vibrational spectroscopy. Thus a good potential of zeolite framework interactions is required in order to distinguish among vibrational peaks originating from the vibrations of the zeolite framework, water molecules, and hydroxyl groups, respectively.

One way to find such a potential is to compare the model-predicted infrared (IR) spectra with the measured IR spectra. Microscopically speaking, all the atoms in a material vibrate around their equilibrium positions, and these vibrations can be used to characterize the structure of the studied material. While not visible, vibrations inside materials can often be excited by absorbing photons with energies in the infrared range. Since vibrations inside materials have characteristic frequencies, depending on the material structure and atomic interactions; and photon energy absorption occurs 


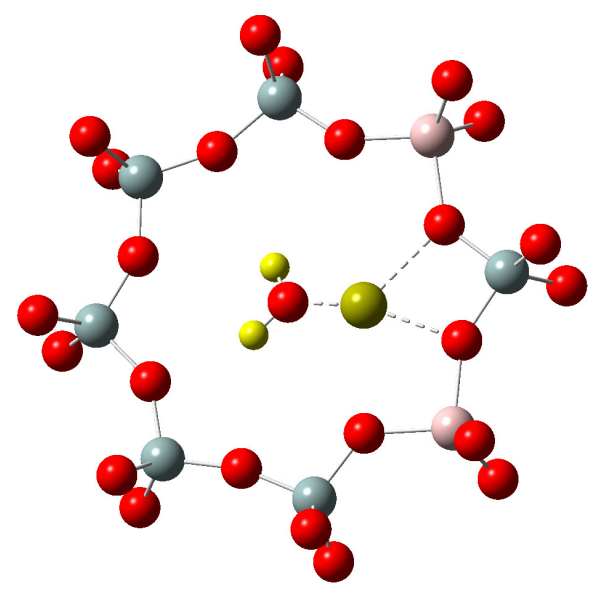

(a) Molecular adsorption

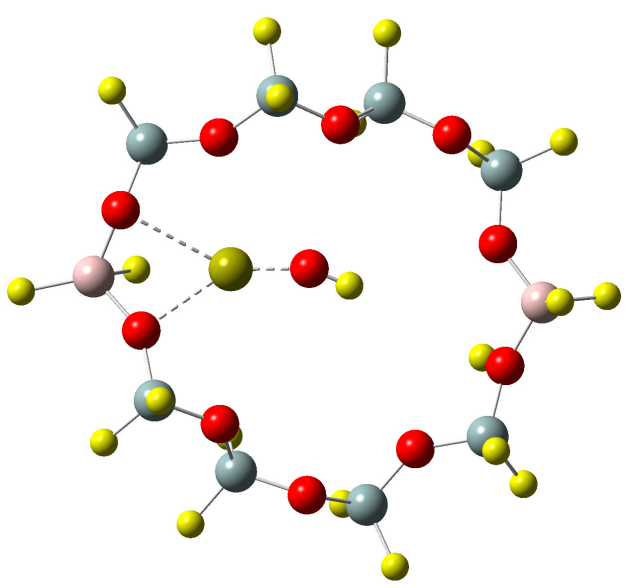

(b) Dissociative adsorption

Figure 1.4. An illustration of water adsorption in zeolites via two different mechanisms: (a) the whole water molecule attaches to the $\mathrm{Zn}^{2+}$ cation in the molecular adsorption mechanism; (b) the water molecule dissociates into a hydroxyl group attaching to the $\mathrm{Zn}^{2+}$ cation, and a leaving proton in the dissociative mechanism.

only when the frequency of the infrared light and the vibration match with each other; excitement of vibrations via absorbing infrared light photon energies will reduce the intensity of the transmitted infrared light at certain frequencies, which gives rise to IR spectra with peaks of varying intensity. Therefore, IR spectroscopy is widely used as a tool to study material structure. For example, Figure 1.5 presents the computed IR spectrum of a methanol solution. The resulted IR spectrum is characteristic of the structure, bonding, and solvation of methanol molecules. A potential that fails to match the model-predicted IR peaks with the corresponding measured IR peaks does not really reproduce the interactions inside the studied material correctly. In the case of zeolites, failing to predict zeolite characteristic IR peaks means that the adopted potential is not capable of describing zeolite framework interactions correctly, even if geometric observables like bond lengths and angles are well-reproduced. This is because model-predicted IR spectra are characteristic not only of zeolite geometries, but also vibrations of the zeolite lattice. 


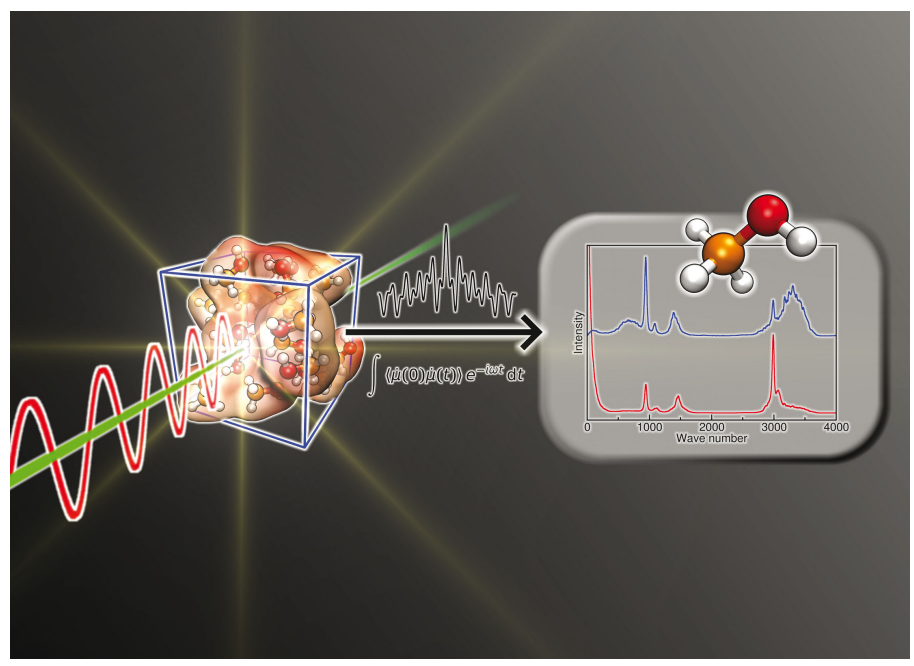

Figure 1.5. An illustration of the application of IR spectroscopy in the characterization of material structures: a laser is used to excite the methanol solution, and the resulted IR spectrum is measured via Fourier transform to give characteristic IR peaks of methanol. Picture from $[17]$

Several potentials that predict siliceous zeolite IR spectra to different levels of accuracy have been developed by other researchers. For example, Ermoshin et al. [18] developed a Generalized Valence Force Field $(\mathrm{GVFF})^{2}$ based on quantum-chemical calculations on a cluster consisting of two $\mathrm{SiO}_{4}$ terminated by $\mathrm{H}$ atoms, and defined the potential parameters in terms of internal coordinates. While success in calculating IR spectra for siliceous sodalite (SOD), faujasite (FAU), and zeolite A (LTA) were achieved in their work, the functional forms used in this potential are complicated, and the cross-interaction terms make it difficult to use this potential in some of the popular simulation packages (see Section 2.1.2.5). Smirnov and Bougeard [19] studied the performance of an effective pair potential (DHFF) and another Simplified Generalized Valence Force Field (SGVFF). The comparison of IR spectra for siliceous FAU showed that the SGVFF is more capable of predicting zeolite IR spectra than the DHFF. A possible reason is that the DHFF potential does not have three-body

\footnotetext{
2 "Force field" is another name of "potential"; "force field" is usually used in the community of physical chemistry, while "potential" is used in the community of chemical physics. To keep consistency, we used "potential" through out this thesis.
} 
angle interactions to constrain the deformation of angles, indicating that the choice of potential is critical and simple potentials may not be able to approximate the zeolite framework interactions satisfactorily. Note that the above valence potentials (GVFF, DHFF, SGVFF) do not include long-range interactions, however, long-range interactions are exactly the interactions between host and guest molecules. This means that long-range interaction parameters will have to be determined if such potentials are to be used in the modeling of zeolite applications. Nicholas et al. [20] developed a more accurate valence potential for siliceous zeolites, extended from the MM2 potential ${ }^{3}$ that includes higher-order energy corrections for three-body interactions. While a good reproduction of silicalite's infrared spectrum was achieved, incorporation of aluminum into this potential in order to be used for the modeling of zeolite applications will be trickier, since two more parameters would be needed to determine for angle interactions. Therefore, we think there is still a strong need for an easily-implemented potential that reproduces zeolite IR spectra satisfactorily. Therefore, this work is aimed to develop such a siliceous zeolite potential.

In the rest of this thesis, Chapter 2 briefly introduces the basics of potentials and their parameterizations as well as the mathematical background of the two IR spectrum-predicting methods adopted in this work. In Chapter 3, two easilyimplemented zeolite potentials, the MZHB potential developed by Sahoo and Nair [21] and the core-shell model developed by Schröder and Sauer [22], are tested by predicting the geometry, bulk modulus, and infrared spectrum of five silica polymorphs. In Chapter 4, a new potential is developed via re-parameterizing and extending the MZHB potential, and the performance of the new potential is tested; Chapter 5 presents a conclusion of this work.

\footnotetext{
${ }^{3}$ The MM2 potential was developed mainly for conformational analysis of hydrocarbons and other small organic molecules
} 


\section{CHAPTER 2}

\section{THEORETICAL BACKGROUND}

\section{$2.1 \quad$ Potentials}

In molecular mechanics and molecular dynamics, potentials are sets of formulas describing the dependence of the potential energy on various internal coordinates, such as bond length, bond angle, and interatomic distance. These formulas help to construct the potential energy surface, which is a multi-dimensional energy hypersurface with respect to each internal coordinate describing the molecular geometry. Accurate molecular potential energies are often obtained by quantum mechanical calculations with large basis sets and high theoretical levels. However, full-quantum mechanical calculations are often limited by the size of the molecule, the acceptable simulation time duration, and memory and storage considerations.

The purpose of potential development is actually to describe the potential energy of the system of interest with a model that only depends on atomic positions, so that forces acting on the atoms can be described without considering electronic effects ex-

plicitly. In this aspect, potentials can be regarded as a fit to the quantum mechanical potential energy surface, to some extent. In fact, one of the widely used potential parameterization strategies is indeed fitting to the quantum mechanically determined potential energy $[18,23,24]$. Note that from the perspective of quantum mechanics, all potential energy arises from the interactions of electrons and nuclei; as such, all the terms that appear in a given potential actually serve the same purpose: that is, to reproduce the electronic interactions of the molecule of interest. 
Table 2.1. Typical components of zeolite potentials categorized by the number of atoms involved.

\begin{tabular}{lll}
\hline Atoms & Potential term & Example \\
\hline \multirow{2}{*}{ Two } & bond & Harmonic bond potential $V=\frac{1}{2} k_{R}\left(R-R_{0}\right)^{2}$ \\
& & Morse potential $D_{e}\left[\left(1-\exp \left(-a\left(R-R_{0}\right)\right)\right)^{2}-1\right]$ \\
\cline { 3 - 3 } & van der Waals & Lennard-Jones potential $\epsilon\left[\left(\frac{r_{m}}{r}\right)^{12}-2\left(\frac{r_{m}}{r}\right)^{6}\right]$ \\
& & Buckingham potential $A e^{-r / \rho}-C r^{-6}$ \\
Three & angle & Harmonic angle potential $V=\frac{1}{2} k_{\theta}\left(\theta-\theta_{0}\right)^{2}$ \\
& & Urey-Bradley $V=\frac{1}{2} k_{\mathrm{UB}}\left(r_{13}-r_{0}\right)^{2}$ \\
\hline \multirow{2}{*}{ Four } & torsion & $\sum_{n} \frac{1}{2} V_{n}\left(1+\cos \left(n \phi-\phi_{0}\right)\right)$ \\
& improper & $V_{\text {improper }}=k_{\Phi}\left(\Phi-\Phi_{0}\right)^{2}$ \\
\cline { 3 - 3 } & angle-angle & $\frac{1}{2} k_{\theta_{1}, \theta_{3}}\left(\theta_{i j k}-\theta_{i j k, 1}\right)\left(\theta_{k j l}-\theta_{j k l, 3}\right)+$ \\
& & $\frac{1}{2} k_{\theta_{1}, \theta_{2}}\left(\theta_{i j k}-\theta_{i j k, 1}\right)\left(\theta_{i j l}-\theta_{i j l, 2}\right)+$ \\
& & $\frac{1}{2} k_{\theta_{2}, \theta_{3}}\left(\theta_{i j l}-\theta_{i j l, 2}\right)\left(\theta_{k j i}-\theta_{k j i, 3}\right)$ \\
\hline
\end{tabular}

Different types of potentials have been developed for various materials and modeling purposes, and the complexities of potentials vary widely according to the purposes. For example, a potential designed for metals [25] could be much more complex than a typical potential designed for proteins [26]. Complexities also depend on specific properties that a potential is used to study for. For example, a typical potential designed for the study of gas molecules adsorption in zeolites may have only a van der Waals interaction term and an electrostatic interaction term defined between zeolite framework atoms and gas molecules/atoms (e.g., Reference 27), whereas a potential designed for the study of zeolite structure will have more interactions among framework atoms besides the van der Waals interactions (e.g., Reference 20). Since the present work focuses on computational studies of zeolites, Table 2.1 lists typical components of potentials that are widely used in zeolite modeling. Categorization is based on the number of atoms involved in the model. Functional forms with more terms have been discussed elsewhere [28, 29]. 


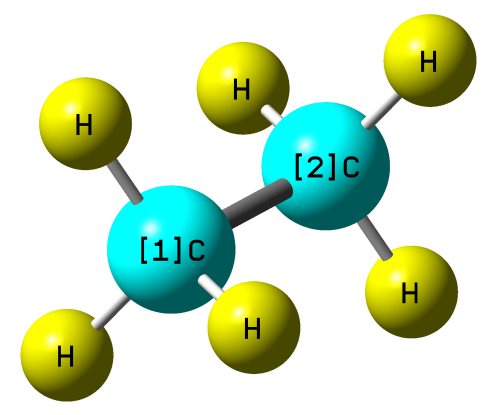

(a) Bond terms

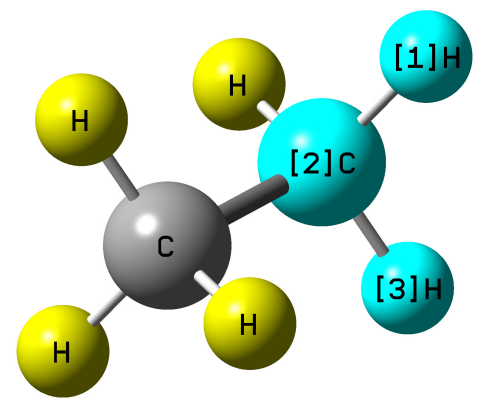

(b) Angle terms

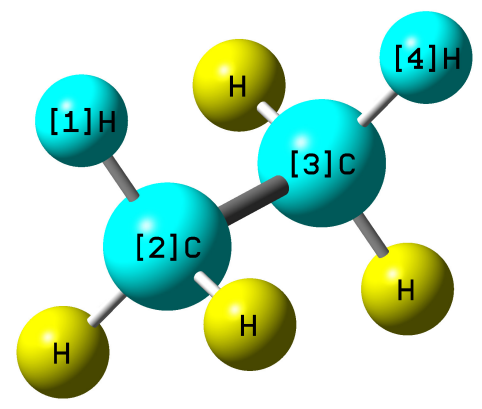

(c) Dihedral terms

Figure 2.1. Illustrations of potential terms with an ethane molecule: (a) $\mathrm{C}-\mathrm{C}$ bond; (b) $\angle \mathrm{HCH}$ angle; (c) dihedral angle for $\mathrm{H}-\mathrm{C}-\mathrm{C}-\mathrm{H}$.

Table 2.2 shows another categorization based on the bonding of the involved atoms. Potential terms in which the involved atoms are required to be bonded together are categorized as valence interaction terms; potential terms in which the involved atoms are not necessarily bonded together are called non-valence interaction terms. Illustrations of the interaction terms are presented in Figures 2.1 and 2.2.

Table 2.2. Typical components of potentials categorized by atomic bonding conditions.

\begin{tabular}{cc}
\hline Potential type & Potential term \\
\hline \multirow{3}{*}{ Valence interactions } & bond term \\
& angle term \\
& torsion term \\
& improper term \\
& cross interaction term \\
\cline { 2 - 2 } Non-valence interactions & van der Waals term \\
& Coulomb interaction term \\
\hline
\end{tabular}

\subsubsection{Potential Classification}

As mentioned in Section 2.1, different types of potentials have been developed for various purposes. Nevertheless, a simple classification of the potentials that are 


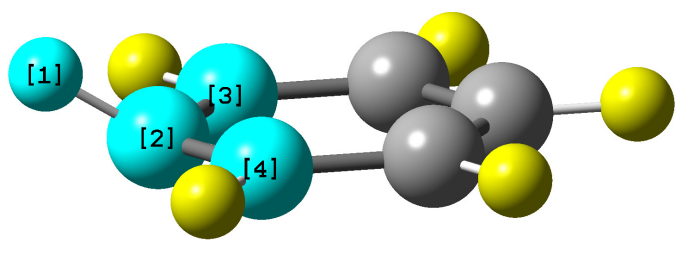

(a) Improper term

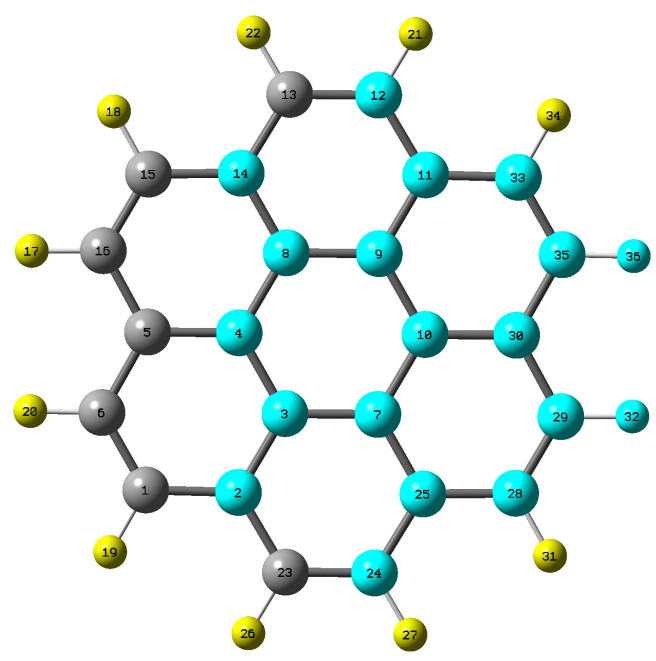

(b) Non-valence terms

Figure 2.2. Illustrations of improper potential and non-valence interactions. (a) the improper potential for atom pair 1-4 is defined to penalize the movement of atom 1 away from the plane formed by atom 2, 3 and 4; (b) an illustration of non-valence interaction terms including the van der Waals interactions and the Coulomb interactions: any atoms within the cutoff radius of atom 10, apart from atom 10 itself, and separated by 4 bonds or more from atom 10 (i.e., atoms that are not highlighted in the picture) interact with atom 10 only via non-valence interactions.

typically used for the modeling of zeolites is discussed below. Generally speaking, there are three types of potentials widely used for the modeling of zeolites, including:

- CHARMM-style potentials [30]

- Valence potentials [18]

- The core-shell model [22]

Brief introductions of these three types of potentials are given individually.

\subsubsection{Valence Potential}

This type of potential is formulated based on a Taylor expansion of the total potential energy with respect to internal degrees of freedom, namely, bond lengths, 
angles, dihedrals, and impropers, as given by Equation (2.1).

$$
\begin{aligned}
V=V_{0} & +\left(\frac{\partial V}{\partial R}\right)_{0}\left(R-R_{0}\right)+\left(\frac{\partial V}{\partial \theta}\right)_{0}\left(\theta-\theta_{0}\right)+\left(\frac{\partial V}{\partial \phi}\right)_{0}\left(\phi-\phi_{0}\right) \\
& +\frac{1}{2}\left(\frac{\partial^{2} V}{\partial R^{2}}\right)_{0}\left(R-R_{0}\right)^{2}+\frac{1}{2}\left(\frac{\partial^{2} V}{\partial \theta^{2}}\right)_{0}\left(\theta-\theta_{0}\right)^{2}+\frac{1}{2}\left(\frac{\partial^{2} V}{\partial \phi^{2}}\right)_{0}\left(\phi-\phi_{0}\right)^{2} \\
& +\left(\frac{\partial^{2} V}{\partial R \partial \theta}\right)_{0}\left(R-R_{0}\right)\left(\theta-\theta_{0}\right)+\left(\frac{\partial^{2} V}{\partial R \partial \phi}\right)_{0}\left(R-R_{0}\right)\left(\phi-\phi_{0}\right) \\
& +\left(\frac{\partial^{2} V}{\partial \theta \partial \phi}\right)_{0}\left(\theta-\theta_{0}\right)\left(\phi-\phi_{0}\right)+\cdots
\end{aligned}
$$

In Equation (2.1), $V$ is a function of the internal coordinates $R, \theta$ and $\phi$, which denote bond length, bond angle, and dihedral angle, respectively. The subscript " 0 " denotes the equilibrium state. Note that in Equation (2.1), the first derivatives are zero since the expansion is performed at the equilibrium state. Neglecting the equilibrium energy $V_{0}$ and third and higher-order terms, replacing the second derivatives with corresponding force constants, one gets

$$
\begin{array}{r}
V=\frac{1}{2} k_{R}\left(R-R_{0}\right)^{2}+\frac{1}{2} k_{\theta}\left(\theta-\theta_{0}\right)^{2}+\frac{1}{2} k_{\phi}\left(\phi-\phi_{0}\right)^{2}+k_{R, \theta}\left(R-R_{0}\right)\left(\theta-\theta_{0}\right) \\
+k_{R, \phi}\left(R-R_{0}\right)\left(\phi-\phi_{0}\right)+k_{R, \theta}\left(\theta-\theta_{0}\right)\left(\phi-\phi_{0}\right)
\end{array}
$$

Equation (2.2) describes a way in which the valence potential terms can be formulated; only atoms that are bonded to each other are considered in the formulation. This functional form is a named potential, the Generalized Valence Force Field (GVFF) $[18,19]$. Note that Equation (2.2) is just a second order Taylor expansion; higher-order terms are also used by researchers in order to obtain more accurate potential energies, especially for the bond-stretching term $(R)$ and angle-bending term 
( $\theta)$ [23], for example,

$$
\begin{gathered}
V_{\text {bond }}=\frac{1}{2} k_{R, 2}\left(R-R_{0}\right)^{2}+\frac{1}{2} k_{R, 3}\left(R-R_{0}\right)^{3}+\frac{1}{2} k_{R_{4}}\left(R-R_{0}\right)^{4} \\
V_{\text {angle }}=\frac{1}{2} k_{\theta, 2}\left(\theta-\theta_{0}\right)^{2}+\frac{1}{2} k_{\theta, 3}\left(\theta-\theta_{0}\right)^{3}+\frac{1}{2} k_{\theta, 4}\left(\theta-\theta_{0}\right)^{4} .
\end{gathered}
$$

While the inclusion of higher-order energy correction terms gives rise to more flexibility to adjust the shape of potential energy surface, the dependencies between model-predicted observables and model parameters also become more complicated as the number of parameters increases, as discussed in Chapter 1. The inclusion of cross interaction terms between bond lengths, angles and dihedrals provides possibilities to better account for the coupling effects between internal degrees of freedom, which makes this type of potential preferable in cases in which subtleties of the potential energy hyper-surface are required, such as the calculation of IR spectra. It is observed that this type of potential generally have good performance in terms of zeolite IR spectra prediction. Several such potentials have been developed $[18,19]$. While computational expenses of valence potentials are very low due to the absence of longrange interactions such as electrostatic interactions and the van der Waals interactions, implementation of valence potentials, such as the GVFF developed by Ermoshin et al. [18] are often tricky, since not all these cross interaction terms are coded in popular MD simulation packages. It also becomes tedious to define these cross interaction terms among individual atoms when the size of the simulated system gets bigger. Another major problem of valence potentials is the difficulty of the parameterization due to the large number of model parameters and the resulting ambiguous dependencies between model parameters and model predictions. Specifically, from the perspective of this work, valence potentials do not include long-range interactions. These interactions are, however, the interactions between the zeolite framework (the host) and reacting species or adsorbates (guests), indicating that long-range interactions 
parameters will need to be determined if such valence potentials are to be used in the modeling of zeolite applications.

\subsubsection{CHARMM-Style Potential}

Unlike valence potentials, most of the potentials used by researchers today for zeolites contain non-valence interaction terms including the van der Waals interactions and electrostatic interactions in order to account for the long-range contributions to the potential energy. Non-valence interactions are often the most time-consuming part in a molecular dynamics (MD) simulation, since the evaluation of non-valence interactions usually requires a long-range summation method [28]. Non-valence interactions are implemented in CHARMM-style potentials. This type of potentials are mainly designed for the modeling of biological systems, including proteins, peptides, lipids, and nucleic acids, as they occur in solution, crystal and membrane environments. The Lennard-Jones parameters, for organic elements like O, C, N, S, H, etc. are listed in a database, and are ready to be used via combination rules, which often offer reasonable initial guesses of model parameters when model parameters are needed to be fine-tuned for specific purposes. This Lennard-Jones parameter database is important, since zeolite frameworks interact with guest molecules via long-range interactions including Lennard-Jones and electrostatic interactions, which makes the CHARMM-style potential preferable than the other two types of potential when the potential is to be adopted for the simulation of catalytic reactions and adsorption. This is actually part of the reasons why we choose to develop a new CHARMM-style potential in Chapter 4. Parameterization procedures of this type of potential are also well-studied [31]. Nevertheless, this type of potential also finds applications in inorganic systems such as zeolites [32, 33]. Typical components of the potential energy described by a CHARMM-style potential are listed in Table 2.3. The total potential energy is given by Equation (2.5), 
Table 2.3. Typical components of a CHARMM-style potential

\begin{tabular}{cc}
\hline Potential type & Potential term \\
\hline \multirow{2}{*}{ Valence potential } & $\begin{array}{c}\text { bond term } \\
\text { angle term } \\
\text { dihedral term } \\
\text { Urey-Bradley }\end{array}$ \\
\cline { 2 - 2 } Non-valence potential & van der Waals term \\
& \\
\hline & \\
$V=V_{\text {bond }}+V_{\text {angle }}+V_{\text {dihedral }}+V_{\mathrm{UB}}+V_{\mathrm{EL}}+V_{\mathrm{vdW}}$,
\end{tabular}

where $V_{\text {bond }}$ and $V_{\text {angle }}$ are harmonic bond-stretching and angle-bending potentials, $V_{\text {dihedral }}$ is defined between atom pairs connected by three bonds, and $V_{\mathrm{UB}}$ is used to account for the coupling effect of bond and angle deformations (see Table 2.1). The electrostatic interactions, $V_{\mathrm{EL}}$, are charge-charge interactions, and $V_{\mathrm{vdW}}$ can be formulated as a Lennard-Jones potential or a Buckingham potential, which will be discussed in Section 2.1.3. The van der Waals (vdW) interactions represent attractive dispersion and repulsive Pauli exclusion between pairs of atoms, and the electrostatic interactions represent Coulomb interactions between pairs of atoms [26]. These nonvalence interactions are sometimes switched off or scaled between valence atom pairs (see Section 2.1.2), as illustrated in Figure 2.3, depending on how the potential is parameterized [30, 34]. If classical mechanical calculations during the parameterization do not include non-valence interactions between atom pairs that are bonded (1-2), pairs separated by two bonds (1-3), and pairs separated by three bonds (1-4), then a 1-4 scale method is often adopted in the implementation of the resulting potential to avoid double counting the interactions between these atom pairs. In the 1-4 scale method, non-valence interactions between atom pairs separated by one or two bonds (1-2 and 1-3) are excluded, and non-valence interactions between atom 


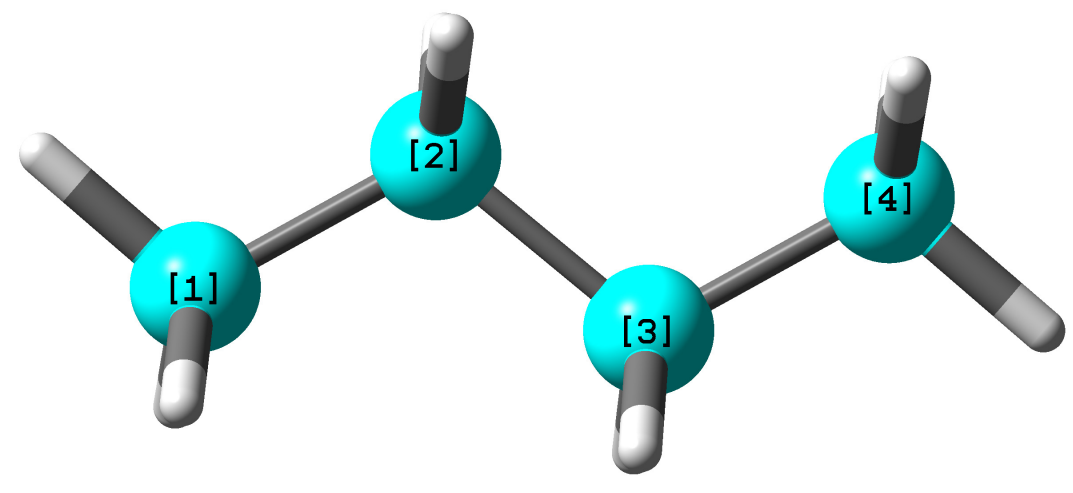

Figure 2.3. An illustration of the $1-4$ scale method: when using a potential of which valence potential terms are parameterized without non-valence interactions included, nonvalence interactions between atom pair (1-2) and (1-3) need to be excluded, and non-valence interactions between atom pair (1-4) are usually scaled, in order to avoid double counting the interactions between these atom pairs.

pairs separated by three bonds (1-4) are scaled by a factor less than unity. In cases in which non-valence interactions are not excluded between valence atom pairs, valence potential terms and non-valence potential terms together represent the local potential energy. Again, Equation (2.2) is a second-order Taylor approximation, thus non-valence interactions between valence atom pairs can be considered as higherorder corrections to the local potential energy, which offers more degrees of freedom to the fitting to the quantum mechanically determined potential energy surface. A brief introduction to valence and non-valence potential components as well as their corresponding popular functional forms is given in Sections 2.1.2 and 2.1.3.

\subsubsection{Core-Shell Model}

The core-shell model is a type of ion pair potential first introduced by Dick and Overhauser for studying alkali halide crystals [35]. It has been since widely used in computational studies of ionic materials [22]. As one of the popular way to include 


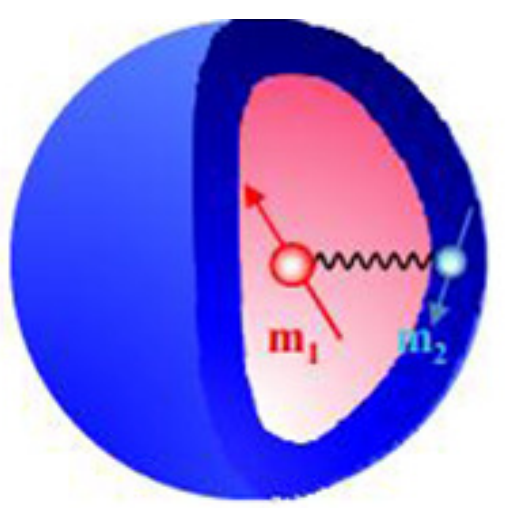

Figure 2.4. An illustration of the core-shell model: a charged atom is separated into a nuclear core and an electron shell, connected via a spring. Charges on the core and shell are constrained by charge conservation. Image from $\mathrm{Ji}$ and $\mathrm{Wu}[36]$.

electron polarization effects in classical mechanical simulations, the main feature of the core-shell model lies in the fact that charged atoms are defined as pairs of nuclear cores and electron shells, connected via springs, as illustrated in Figure 2.4. Usually, the partial charges of the cores and shells are constrained by charge conservation. While the charges on the core and the shell are fixed, polarization effects are accounted by changing the separation distance between the core and the shell. A typical formulation of the total potential energy described by a core-shell model is given by Equation (2.6),

$$
\begin{gathered}
V=V_{\text {short-range }}+V_{\mathrm{EL}}+V_{\text {core-shell }}+V_{\text {angle }} \\
V_{\text {core-shell }}=\frac{1}{2} k_{s} r^{2}
\end{gathered}
$$

where $V_{\text {short-range }}$ denotes the pair-wise two-body interactions, such as the LennardJones or the Buckingham interactions, and $V_{\text {core-shell }}$ arises from the atom's selfpolarization. Note that electrostatic interactions are usually excluded between the core and the shell of the same pair. 


\subsubsection{Valence Potential}

\subsubsection{Bond Potentials}

Three types of widely-used functional forms have been developed to describe the interactions between bonded atom pairs, including:

Harmonic bond potential This type of bond potential is based on the harmonic approximation (see Table 2.1), in which the energy increases quadratically with respect to the deviation of internal coordinates against their equilibrium values. It is easy to implement due to the simple functional form and low number of parameters, but one major shortcoming is that this bond potential requires small bond length deviations, otherwise, the harmonic approximation will break down. Thus, the harmonic bond potential is a non-reactive potential, and is not appropriate in the simulation of chemical reactions, in which bonds are allowed to break and form during the simulation.

Polynomial bond potential As given by Equation (2.3), higher-order terms can be added to the harmonic potential, which gives the ability to account for anharmonicity associated with the potential energy curve. However, more terms also means more parameters to fit, requiring more target data.

The Morse potential The Morse potential [37-39] is slightly more realistic than the harmonic bond potential due to its functional form (see Table 2.1), where the three parameters $D_{e}, R_{0}$ and $a$ have specific physical meanings:

$\boldsymbol{D}_{\boldsymbol{e}}$ Well depth, related to the dissociation energy of the bond $\boldsymbol{R}_{\mathbf{0}}$ Equilibrium bond length

$\boldsymbol{a}$ Strength of the bond, which controls the shape of the energy curve 


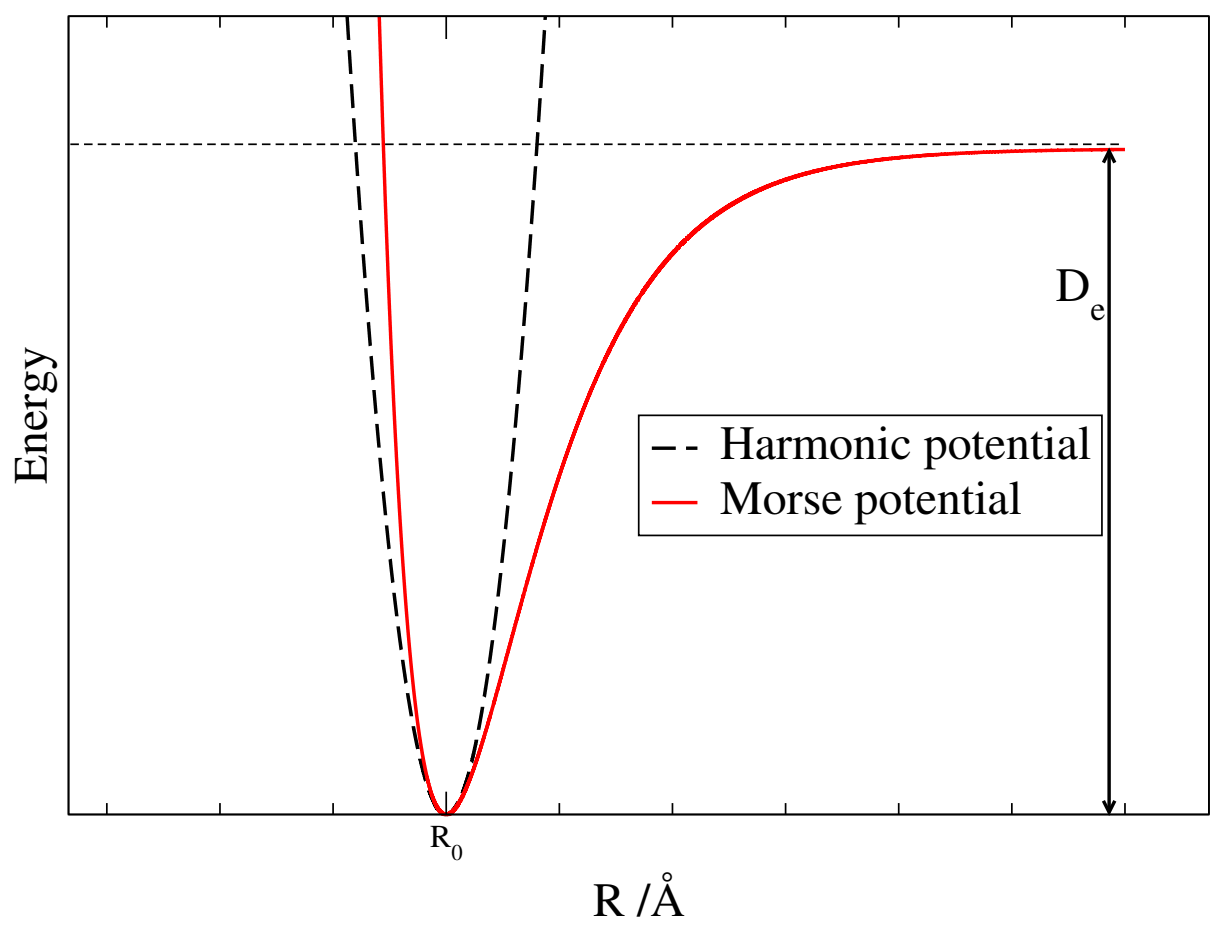

Figure 2.5. A graphical comparison of the harmonic bond potential and the Morse potential: while the prediction of the Morse potential is close to the prediction of the harmonic potential when the distance between the two bonded atoms is within a small range around the equilibrium bond length, it deviates significantly from that of the harmonic potential when the separation distance between the bonded atoms becomes large.

The bond strength is typically related to the well depth, $D_{e}$, and the bond force constant, $k_{R}$, by Equation (2.7),

$$
a=\sqrt{\frac{k_{R}}{2 D_{e}}} .
$$

Graphically, one can easily tell from Figure 2.5 that under small bond length deviation, the Morse potential can be replaced with the harmonic potential without significant errors; however, once the bonded length deviation becomes sufficiently large, then the Morse potential deviates from the harmonic potential and better reflects the nature of chemical bonds since the dissociation of the bond is now possible. 


\subsubsection{Angle Potentials}

These potential terms describe the interactions between three atoms, $i, j$ and $k$, or two bonds with a common atom. Numerous functional forms have been developed for three-body potentials; we give a brief introduction here to the functional forms that are most widely used in the simulation of zeolites, readers for more detailed descriptions and various types of three-body interactions please see Reference [29].

Two physical representations of three-body potentials are commonly used [29], which give rise to two categories of three-body potentials:

Covalent potentials From the perspective of covalent potentials, three-body interactions arise from the repulsion of two bonded atom pairs. Two functional forms are widely used to penalize angle deviation against the equilibrium angle, including:

Harmonic angle potential The most intuitive functional form for three-body interactions, see Table 2.1.

Polynomial angle potential Higher order polynomials such as Equation (2.4) are also used to account for the anharmonicity associated with the shape of the potential energy curve.

Ionic potentials On the other hand, from the perspective of ionic potentials, threebody interactions arise from the interactions of the induced dipoles of the three involved atoms [29]. For example

The Axilrod-Teller potential[40] This type of three-body potential is obtained from third order perturbation theory,

$$
V_{\text {angle }}=D\left(\frac{1+3 \cos \left(\theta_{k i j}\right) \cos \left(\theta_{i j k}\right) \cos \left(\theta_{j k i}\right)}{R_{i j}^{3} R_{i k}^{3} R_{j k}^{3}}\right),
$$


where $D$ is a constant of the order $I \alpha^{3}$ with $I$ being the ionization energy, and $\alpha$ being the average atomic polarizability; $R_{i j}$ denotes the bond length between atom $i$, and $j$. This type of three-body potential is widely used in fluid simulations $[41,42]$.

\subsubsection{Torsional Potentials}

Potential energies arising from torsional angles are another intramolecular energy component in a potential, which can be regarded as the repulsion energy originating from unfavorable molecular configurations. Recall that in Equation (2.2), torsional potentials are derived in harmonic function form. In fact, torsional potentials, however, are quite different compared with bonded potentials and angle potentials [43]. The first difference is that unlike the small deviations in bond potentials and angle potentials, the torsional angle deviations can be large due to low energy barriers. Another major difference originates from the rotational periodicity of torsional angles, which means that torsional potentials will have several energy minima (how many depends on the specific molecule being studied). Thus simple Taylor expansion cannot efficiently represent torsional potentials. Instead, the most widely used functional form for torsional potentials is a Fourier expansion,

$$
V_{\text {dihedral }}=\sum_{n} \frac{1}{2} V_{n}\left(1+\cos \left(n \phi+\phi_{0}\right)\right) .
$$

In Equation (2.9), $\phi$ and $\phi_{0}$ denote the torsional phase angle and the equilibrium phase angle, respectively; $n$ and $V_{n}$ denote the rotational periodicity and the energy barrier, respectively. The number of terms depends on the complexity of the torsional energy curve. In most cases, three terms are sufficient to describe an energy curve [23]. 


\subsubsection{Improper Potentials}

Another type of potential that involves four atoms is the improper potential, also called the out-of-plane potential. Out-of-plane potentials are mostly used to penalize the movement of one atom leaving the plane formed by the other three atoms. It can also be regarded as a penalty applied when the angle formed by two planes deviates from its equilibrium value. The functional form is given in Table 2.1. The value of the equilibrium angle $\Phi_{0}$ depends on molecular geometry. In the case of a planar molecule, $\Phi_{0}$ is set to be zero, and the functional form is reduced to Equation $(2.10)[43,44]$,

$$
V_{\text {improper }}=k_{\Phi} \Phi^{2}
$$

\subsubsection{Cross Interactions}

From the Taylor expansion of total potential energy in Equation (2.2), cross interaction terms are easy to understood. The functional forms of these cross interaction terms are combinations of individual bond, angle, and torsion terms, reflecting the coupling effects of internal degrees of freedom, which cannot be accounted via individual potential terms. Take a single water molecule as an example: bond and angle potentials can only account for the energy contributions of $\mathrm{H}-\mathrm{O}$ bond-stretching motion and $\angle \mathrm{HOH}$ angle-bending motion, respectively. However, note that the change of the $\mathrm{H}-\mathrm{O}$ bond lengths will change the interatomic distance between the two $\mathrm{H}$ atoms, which, from the covalent perspective, will alter the repulsion between the two $\mathrm{H}$ atoms, hence change the $\angle \mathrm{HOH}$. Qualitatively speaking, in the case of $\mathrm{H}-\mathrm{O}$ bond symmetric stretching, one would expect that $\angle \mathrm{HOH}$ increases. Such coupling effects

are also expected between other internal degrees of freedom. Table 2.4 lists some cross interaction terms that are widely used in valence potentials. 
Table 2.4. Typical cross interaction terms used in valence potentials.

\begin{tabular}{ccc}
\hline Cross potential & Functional form & Reference \\
\hline Bond-bond cross & $\sum_{R_{1} \neq R_{2}} k_{R_{1}, R_{2}}\left(R_{1}-R_{1,0}\right)\left(R_{2}-R_{2,0}\right)$ & {$[18,23,44]$} \\
Angle-angle cross & $\sum_{\theta_{1} \neq \theta_{2}} k_{\theta_{1}, \theta_{2}}\left(\theta_{1}-\theta_{1,0}\right)\left(\theta_{2}-\theta_{2,0}\right)$ & {$[18,23,44]$} \\
Bond-angle cross & $\sum_{R} \sum_{\theta} k_{R, \theta}\left(R-R_{0}\right)\left(\theta-\theta_{0}\right)$ & {$[18,23,44]$} \\
Angle-angle-torsion cross & $\sum_{\theta_{1}} \sum_{\theta_{2}} \sum_{\phi} k_{\theta_{1}, \theta_{2}, \phi}\left(\theta_{1}-\theta_{1,0}\right)\left(\theta_{2}-\theta_{2,0}\right) \cos \phi$ & {$[23,44]$} \\
\hline
\end{tabular}

\subsubsection{Urey-Bradley Potential}

Another way to include bond-angle coupling effect is to use the Urey-Bradley potential (see Table 2.1), where $r$ is the internuclear distance between two terminal atoms in an angle. As a standard component in the CHARMM potential family [30], this potential term helps distinguish subtleties in vibrational spectra. For example, it is said that this Urey-Bradley term is necessary to distinguish the symmetric and asymmetric $\mathrm{Si}-\mathrm{O}$ stretching vibrational frequencies [20].

\subsubsection{Non-valence Potential}

\subsubsection{Coulomb Interactions}

Electrostatic interactions are generally evaluated with Coulomb's Law,

$$
\begin{aligned}
f_{i j} & =\frac{1}{4 \pi \epsilon_{0}} \frac{q_{i} q_{j}}{r_{i j}^{2}} \\
V_{i j} & =\frac{1}{4 \pi \epsilon_{0}} \frac{q_{i} q_{j}}{r_{i j}},
\end{aligned}
$$

where $\epsilon_{0}$ is the vacuum permittivity, and $q_{i}$ and $q_{j}$ are charges of atom $i$ and $j$. Written in vector form, then the total electrostatic force felt by atom $i$ can be expressed as

$$
\mathbf{f}_{\mathbf{i}}=\sum_{j \neq i}^{N} \frac{1}{4 \pi \epsilon_{0}} \frac{\left(\mathbf{r}_{\mathbf{i}}-\mathbf{r}_{\mathbf{j}}\right) q_{i} q_{j}}{\left|r_{i}-r_{j}\right|^{3}}
$$


Equation (2.12) is used to calculate the electrostatic force felt by atom $i$ applied by all the remaining atoms in the simulated system for a given atomic charge distribution. Generally speaking, there are several approaches for the determination of atomic charge distributions during MD simulations, including:

- Fixed point charges

- Point dipole method [45]

- Charge equilibrium method $[46,47]$

- Drude model $[48,49]$

Brief introductions to these methods are discussed in the next several sections.

2.1.3.1.1 Fixed point charges The most intuitive way to determine atomic charges is using fixed point charges, in which the atomic charges are specified as model parameters at the beginning of an MD simulation and kept constant during the simulation. Note that besides using formal charges for atomic species, partial charges are also widely used in molecular mechanics and molecular dynamics simulations. Table 2.5 lists partial charges for silicon used in various models in the literature. Note that electron-electron polarization effects, under the scheme of fixed point charges, are simply accounted by a dielectric constant $k$, where the total permittivity is $\epsilon=$ $k \epsilon_{0}$; this is an inherent deficiency of this method to account for the effects of the environment on atomic charges [50]. However, due to the simple concept and easilyimplemented feature, the fixed point charges method is widely used in simulations, especially for atomistic simulations with large numbers of particles involved.

2.1.3.1.2 Point Dipole Method This method starts from the consideration that the electron cloud surrounding atoms can be deformed to generate induced dipole moments under the existence of an electric field. Application of this method in 
Table 2.5. Example of partial charges for Si atoms used in classical MD simulations in the literature.

\begin{tabular}{lll}
\hline Si Charge (e) & Method & Reference \\
\hline 0.70 & Fit to quantum mechanical results & {$[27]$} \\
1.08 & $\begin{array}{l}\text { Fit to minimum interacting action energies and ge- } \\
\text { ometries between a water molecule and chemical } \\
\text { groups of the studied cluster }\end{array}$ & \\
& $\begin{array}{l}\text { Fit to quantum mechanical calculated cluster ge- } \\
\text { ometries }\end{array}$ & {$[20]$} \\
1.10 & $\begin{array}{lll}\text { Rounded from electronegativity } \\
\text { method }\end{array}$ & \\
& Half of the formal charge & {$[19]$} \\
2.00 & Formal charge & {$[27]$} \\
\hline .00 & & \\
\hline
\end{tabular}

modeling of zeolites have been attempted by Kermode et al. [45] and by Tangney and Scandolo [51]. Besides the interactions between point charges, the interactions between charge-dipole and dipole-dipole are also accounted, which give rise to an electrostatic energy by Equation (2.13)

$$
V_{\mathrm{EL}}=\sum_{i \neq j}\left(V_{\mathrm{c}-\mathrm{c}}+V_{\mathrm{c}-\mathrm{d}}+V_{\mathrm{d}-\mathrm{d}}\right)
$$

where $V_{\mathrm{c}-\mathrm{c}}$ denotes the charge-charge interactions, given by Equation $(2.11), V_{\mathrm{c}-\mathrm{d}}$ and $V_{\mathrm{d}-\mathrm{d}}$ denote the charge-dipole and dipole-dipole interactions, given by Equations (2.14) and (2.15),

$$
\begin{gathered}
V_{\mathrm{c}-\mathrm{d}}=\frac{Z_{i} e}{4 \pi \epsilon_{0}} \frac{\mathbf{r}_{\mathbf{i j}} \boldsymbol{\mu}_{\mathbf{j}}}{r_{i j}^{3}}, \\
V_{\mathrm{d}-\mathrm{d}}=\frac{1}{4 \pi \epsilon_{0} r_{i j}^{3}}\left[\boldsymbol{\mu}_{\mathbf{i}} \boldsymbol{\mu}_{\mathbf{j}}-3 \frac{\left(\boldsymbol{\mu}_{\mathbf{i}} \mathbf{r}_{\mathbf{i}}\right)\left(\boldsymbol{\mu}_{\mathbf{j}} \mathbf{r}_{\mathbf{i j}}\right)}{r_{i j}^{2}},\right]
\end{gathered}
$$

where $Z_{i}$ and $e$ denote the atomic number of atom $i$ and the elementary charge, respectively; $\mathbf{r}_{\mathbf{i j}}$ and $r_{i j}$ denote the displacement vector from atom $j$ to $i$ and the its scalar value; $\boldsymbol{\mu}_{\mathbf{j}}$ denotes the dipole moment at position of atom $j$. Simple schematic 


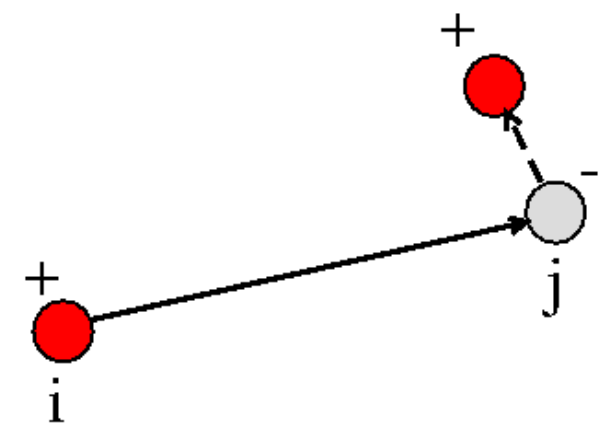

(a) Charge-dipole

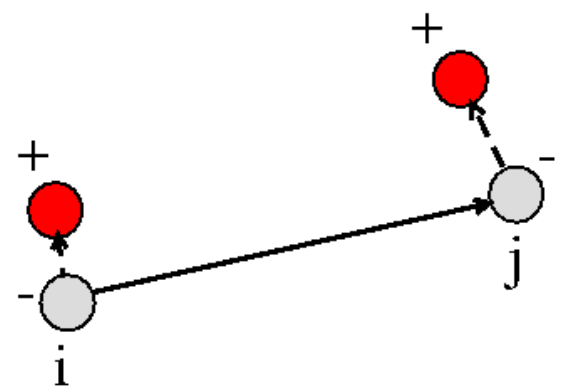

(b) Dipole-dipole

Figure 2.6. Illustrations of charge-dipole and dipole-dipole interactions: (a) a chargedipole interaction schematic diagram; (b) a dipole-dipole interaction schematic diagram.

diagrams of the charge-dipole and dipole-dipole interactions are given in Figure 2.6. Readers desiring detailed mathematical derivations of $V_{\mathrm{c}-\mathrm{d}}$ and $V_{\mathrm{d}-\mathrm{d}}$ are directed to the work of Tangney and Scandolo [51] and Yamamoto [52]. Note that the electrostatic energy, $V_{\mathrm{EL}}$, is not equal to the energy of the induced dipole system, $V_{\text {sys }}^{\text {induced}}$; instead, the energy of the induced dipole system is expressed as

$$
V_{\mathrm{sys}}^{\text {induced }}=V_{\mathrm{EL}}+V_{\text {polar }}
$$

where $V_{\text {pol }}$ denotes the energy required to polarize the particles. In the work of Berendsen et al. [53], the energy of the induced dipole system $V_{\text {sys }}^{\text {induced }}$ is determined by introducing a charge parameter $\lambda$ that is integrated from 0 to 1 ,

$$
V_{\mathrm{sys}}^{\text {induced }}=\sum_{i} \int_{0}^{1} \lambda U_{\mathrm{EL}, i} q_{i} d \lambda=\sum_{i} \frac{1}{2} q_{i} U_{\mathrm{EL}, i}
$$

where $U_{\mathrm{EL}, i}$ denotes for the electrostatic potential at position $i$, arising from point charges and induced dipoles. This formulation of the $V_{\text {sys }}^{\text {induced }}$ comes from the physical meaning of Equation (2.16): given the electrostatic potential $U_{\mathrm{EL}, i}$ at position of 
atom $i$, the system is first charged with point charges $q_{i}$, each with charge parameter $\lambda$; integration gives the system energy $V_{\text {sys }}^{\text {induced }}$. Then the point charges are allowed to be polarized to form dipoles, which gives the energy $V_{\mathrm{EL}}$, and the amount of energy required to achieve this polarization $V_{\text {polar }}$. In the case of isotropic polarizability, the polarization energy $V_{\text {pol }}$ is evaluated to be

$$
V_{\text {polar }}=\frac{1}{2} \sum_{i} \frac{\boldsymbol{\mu}_{\mathbf{i}}^{2}}{\alpha_{i}}
$$

This is obtained with the fact that at equilibrium, the change of $V_{\text {sys }}^{\text {induced }}$ with respect to the change of dipoles is zero.

2.1.3.1.3 Charge Equilibrium Method This method takes the electron polarizability into consideration by redistributing atomic charges according to the instantaneous geometries of the simulated system and the experimental properties of the involved atomic species at each time step. The total electrostatic interaction energy is given by

$$
V_{\mathrm{EL}}=\sum_{i=1}^{N}\left(V_{i, 0}+\chi_{i, 0} q_{i}+\frac{1}{2} J_{i i, 0} q_{i}^{2}\right)+\sum_{i<j} q_{i} q_{j} J_{i j}
$$

where $V_{i, 0}$ is the energy of the charge neutral atom $i$, where a subscript "0" denotes charge neutrality; $N$ is the total number of atoms; $\chi_{i, 0}$ and $J_{i j}$ denote the electronegativity of the charge neutral atom $i$ and the Coulomb interaction energy between unit charges; and the physical meaning of $J_{i i, 0}$ is the Coulomb repulsion between two electrons in the same electronic orbital, known as the idempotential [46] or atomic hardness [50]. Since the chemical potential of an electron gas surrounding its nucleus is the negative of the Mulliken electronegativity of the isolated particle, given by [47]

$$
\mu_{i}=-\chi_{i}=-e \frac{\partial V_{\mathrm{EL}}}{\partial q_{i}}
$$


The chemical potential of atom $i$ can then be expressed as

$$
\mu_{i}=-e\left(\chi_{i, 0}+J_{i i, 0} q_{i}+\sum_{i \neq j} J_{i j} q_{j}\right),
$$

At equilibrium, the electrochemical potentials of each atom are equal, which leads to $N-1$ equations for a system of $N$ atoms, plus charge conservation - that is, the sum of any changes in particle charges is zero, that is,

$$
\sum_{i}^{N} d q_{i}=0
$$

This gives $N$ coupled equations in $N$ variables. Note that in the last term of Equation (2.19), different charge distribution functions yield different Coulomb interaction models with varying levels of complexity. Some commonly used charge distribution functions are Dirac delta functions, which correspond to point charges, and Slatertype $1 s$ orbitals (spherically-symmetric, normalized exponential functions) [46, 54]. Readers desiring more detail are directed to References 46, 54 .

2.1.3.1.4 Drude Model The Drude Model introduces polarizability of particles other than hydrogen via massless auxiliary particles (known as Drude particles) carrying charge and attached to physical particles with harmonic springs [55], as illustrated in Figure 2.4. The charges of the physical particles and the Drude particles are constrained by the charge conservation law,

$$
q_{i}+q_{i, D}=q_{i}^{\prime},
$$

where $q_{i}$ and $q_{D_{i}}$ denote the charges of the physical particle $i$ and Drude particle $D_{i}$, respectively, and $q_{i}^{\prime}$ denotes the charge of particle $i$ without using the Drude model. Note that the charge of the physical particles and Drude particles are both fixed in 
this case; the polarization effect arises from the change of the separation distance between the physical-Drude particle pairs. This method is essentially the same as the core-shell model described in Section 2.1.1.3 - the difference in nomenclature mainly originates from the two communities that use these two models [56]. The Drude model has been widely used in polarized water [49, 55, 57, 58] and organic compounds $[48,59]$, while the core-shell model is mostly applied in the study of solid state materials $[60,61]$. The Drude model can be implemented in two ways, including:

Adiabatic method using this method, the system potential energy $V$ is given by

$$
V=V_{\text {polar }}(\mathbf{d})+V_{\mathrm{EL}}(\mathbf{r}, \mathbf{d})+V_{\text {else }}
$$

In Equation (2.24), $V_{\text {polar }}$ denotes the energy due to the harmonic spring potential between each physical-Drude particle pair, which depends on the separation distance between particles in the pair $\mathbf{d} ; V_{\mathrm{EL}}(\mathbf{r}, \mathbf{d})$ denotes the electrostatic interaction energy contributed by all the charged particles except the physical and Drude particles belonging to the same pair, which is a function of both physical particles' positions $\mathbf{r}$ and pair separations $\mathbf{d}$; $V_{\text {else }}$ stands for other potential energy components. The adiabatic method requires that during each time step of an MD simulation, the potential energy is minimized with respect to the positions of Drude particles while the physical particles' positions are fixed; that is,

$$
\frac{\partial V_{\text {polar }}(\mathbf{d})}{\partial \mathbf{d}_{\mathbf{i}}}+\frac{\partial V_{\mathrm{EL}}(\mathbf{r}, \mathbf{d})}{\partial \mathbf{d}_{\mathbf{i}}}=0
$$

holds for every atom $i$. Solving Equation (2.25) gives a self-consistent total potential energy.

Extended Lagrangian method Another way to implement the Drude model is using an extended Lagrangian, which considers the Drude particles as additional degrees of freedom $[48,55]$. In this case, each Drude particle $D_{i}$ is assigned 


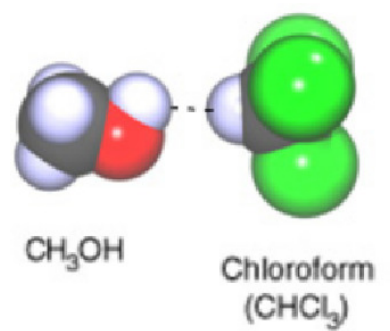

(a)

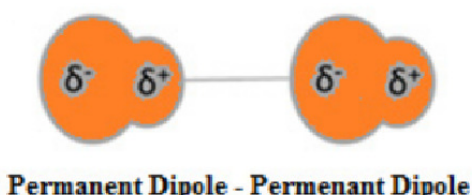

(d)

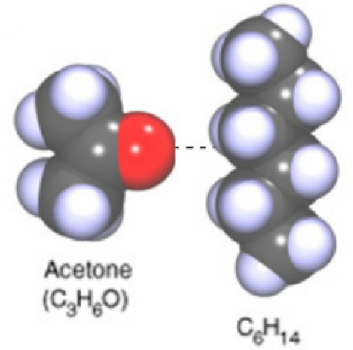

(b)

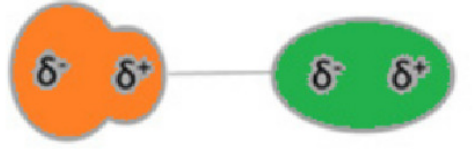

Permanent Dipole - Induced Dipole

(e)

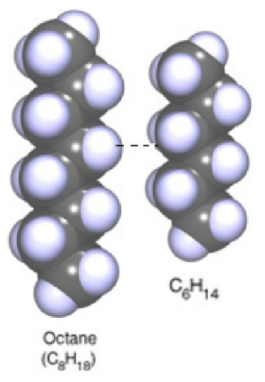

(c)

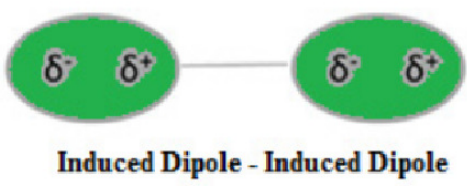

(f)

Figure 2.7. Illustrations of van der Waals interactions. Image adopted from Leite et al. [65]

with a small fraction of mass of the corresponding physical particle $i$ under the constraint of mass conservation. Therefore, for both physical particles and Drude particles, a similar variant of Lagrange's equation of motion applies. This way of implementing the Drude model in classical MD simulations has been documented in the literature; readers interested in details are directed to Lamoureux and Roux [55] and Jiang et al. [58].

\subsubsection{2 van der Waals Interactions}

Another important long-range non-valence interaction is the van der Waals interaction. Van der Waals interactions are important in the explanation of many phenomena such as adhesion [62,63], physical adsorption [64]. The theory of van der Waals interactions has resulted in a set of forces containing three contributions, all proportional to $r^{-6}$, arising from different physical origins [65].

The first contribution to the van der Waals force is the Keesom force, named after Willem Hendrik Keesom [65]. It arises from the attraction between permanent dipoles, 
such as water molecules. Therefore, for molecules for which the electronegativities of neighboring atoms differ significantly, the Keesom force exists. On the other hand, the Keesom force is not applicable to atoms, since atoms cannot have permanent dipole moments. Figures $2.7 \mathrm{a}$ and $2.7 \mathrm{~d}$ illustrate the permanent dipole interaction between two polar molecules, $\mathrm{CH}_{3} \mathrm{OH}$ and $\mathrm{CHCl}_{3}$. Since the result of the permanent dipole-dipole interaction is the reorientation of the involved molecules, the Keesom force actually describes the "orientation effect" [66].

The second part of the van der Waals force is called the Debye force or induction force [65], and it arises from the interactions between permanent dipoles and induced dipoles. The Debye force is weaker than the Keesom force because the magnitude of an induced dipole moment of a charge-neutral atom is typically smaller than that of a permanent dipole moment. As is discussed in Section 2.1.3.1, induced dipole moments arise from the electric field generated by point charges, permanent dipoles, and other induced dipoles at the position at which the atom of interest is located, and acts upon the atom's polarizability. An example of the Debye force, shown in Figures $2.7 \mathrm{~b}$ and $2.7 \mathrm{e}$, show that as a polar molecule, such as acetone, gets close to a non-polar molecule, such as hexane, it deforms the electron cloud of the hexane molecule to form an induced dipole moment aligned opposite to the orientation of the permanent dipole, causing mutual attraction between the two molecules. Like the Keesom force, the Debye force is also not applicable to pairs of atoms for the same reason.

The third component of the van der Waals force is the dispersion force, also known as the London force [66], which arises from the interactions among fluctuating induced dipoles. The polarizability of an atom gives rise to the ability of the atom to be polarized as long as an applied external electric field, or an internal electric field generated by other atoms within the charge-neutral molecule itself, exists. A typical picture of the London forces, shown in Figures 2.7c and 2.7f, depict two non-polar 
charge-neutral molecules attracted by each other due to the fluctuating non-trivial induced dipoles. Since all materials are polarizable to different extents, the London forces is much more prevalent than the Keesom and Debye forces [65].

The above three contributions of the van der Waals interactions can be written into one formula describing the pair-wise the van der Waals force.

$$
V_{\mathrm{vdW}}=\frac{C_{\text {orientation }}+C_{\text {induction }}+C_{\text {dispersion }}}{r^{6}},
$$

An intuitive way to understand why all the three components of the van der Waals interactions are proportional to $r^{-6}$ based on the fact that the electric field generated at position $\mathbf{r}_{\mathbf{i}}$ by a dipole at position $\mathbf{r}_{\mathbf{j}}$ is given by

$$
\mathbf{E}_{\mathbf{i}}=\sum_{i \neq j} \frac{1}{4 \pi \epsilon_{0}} \frac{\mathbf{r}_{\mathbf{i j}} \boldsymbol{\mu}_{\mathbf{j}}}{r_{i j}^{3}}
$$

according to Equation (2.14). With this electric field, an atom at position $\mathbf{r}_{\mathbf{i}}$ will be polarized to form a dipole moment via its polarizability $\alpha_{i}$,

$$
\boldsymbol{\mu}_{\mathbf{i}}=\alpha_{i} \mathbf{E}_{\mathbf{i}},
$$

which is also of the order $r_{i j}^{-3}$. Therefore, dipole-dipole interactions, regardless of permanent dipoles or induced dipoles, defined by

$$
V_{\mathrm{d}-\mathrm{d}}=-\mathbf{E}_{\mathbf{i}} \cdot \boldsymbol{\mu}_{\mathbf{i}},
$$

have the order $r_{i j}^{-6}$. Readers wishing further details are directed to London [66]. A more systematic interpretation of the origin of the van der Waals forces based on the second order perturbation theory in quantum mechanics has been discussed by Jeziorski et al. [67]. 
In specific calculations, the van der Waals interactions are often implemented along with a repulsive term resulting from electron-overlap and Pauli exclusion. Two major types of functional form, the Buckingham potential and the Lennard-Jones potential, are commonly used:

Buckingham Potential The functional form of this model is given in Table 2.1, in which an exponential is used for the repulsive term. This pair-wise energy is inaccurate when the interatomic distance is small: the exponential goes to a finite number as $r_{i j}$ approaches 0, which makes no sense for electrons and nuclei at close range. Care must therefore be taken to make sure that the interatomic distance is large enough to avoid this feature when the Buckingham potential is used.

The Lennard-Jones Potential A more widely-used functional form is the LennardJones potential (see Table 2.1), where a term proportional to $r^{-12}$ is used for repulsions. The introduction of the $r^{-12}$ term is done for simplicity and to reduce the computational expense, since it is easier to evaluate a repulsion term proportional to $r^{-12}$ once the attraction term proportional to $r^{-6}$ is evaluated. The parameters of the Lennard-Jones potential have physical interpretations, as shown in Figure 2.8; $\epsilon$ and $r_{m}$ denote the energy well depth and the minimumenergy internuclear distance, respectively.

\subsubsection{Potential Parameterization}

Functional forms themselves do not lead to potentials: it is the combination of reasonable functional forms and well-derived parameter sets that gives rise to the ability and accuracy of that potential when applied to the objects for which it is designed. Therefore potential parameterization becomes another major aspect in the computational studies of various materials via classical mechanical potentials. Typical procedures fit parameters to quantum mechanical target data based on small 


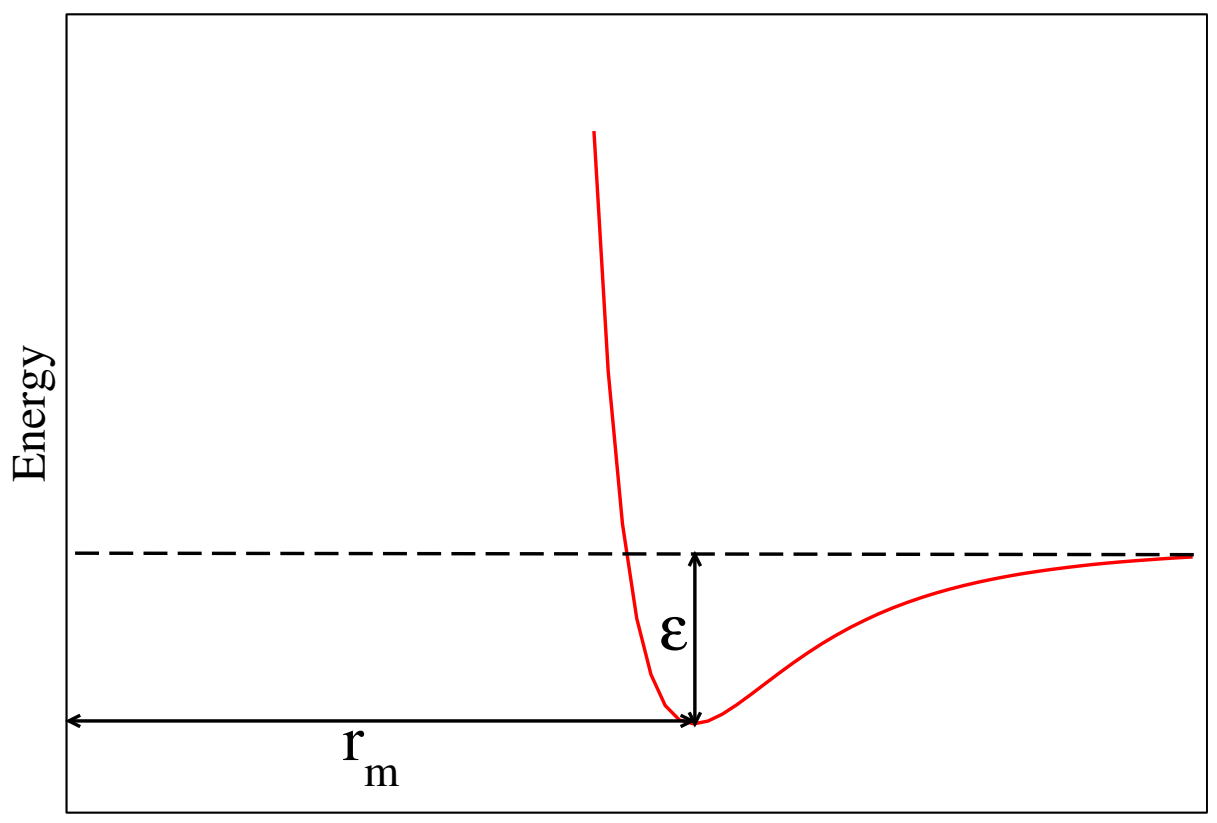

Distance

Figure 2.8. An illustration of the Lennard-Jones potential. The energy depth, $\epsilon$, and minimum-energy internuclear distance, $r_{m}$, are indicated.

characteristic clusters or experimental observables, depending on the functional forms to be parameterized.

The general procedures of potential development have been reasonably well-documented in the literature $[68,69]$, including parameterization methods for most of the functional forms discussed in Sections 2.1.2 and 2.1.3. Due to the strong coupling between model parameters, changing one parameter will require all the other parameters to be adjusted. Nevertheless, a typical parameterization procedure for a CHARMM-style potential with fixed point charges is given by Mayne et al. [31]. A brief walk-through is given below; readers desiring more detailed explanations are directed to Reference 31.

\subsubsection{Bond and Angle Potential Parameterization}

Bond and angle potentials are closely related to objects' geometries and vibrational spectra. Typical quantum mechanical target data based on characteristic small clus- 
ters include $a b$ initio potential energy [23, 70], Hessian matrix [18, 31], or other data related to the second derivatives of the potential energy with respect to coordinates, such as vibrational modes [20,71]. Experimental observables include crystallographic data, such as average bond lengths and angles [71], lattice parameters [21], and mechanical properties such as bulk moduli [21].

\subsubsection{Charge Parameterization}

Two widely used methods to determine atomic partial charges have been developed, including the quantum mechanical potential surface method implemented in AMBER [34], and the supramolecule method implemented in CHARMM [68].

Potential Energy Surface Method The main idea of this method is to determine the atomic charges via minimizing an objective function, which represents the difference between potential energies as calculated from quantum mechanics and classical mechanics. The fitting is carried out by the Lagrange multiplier method, given by Equation (2.30),

$$
y\left(q_{1}, q_{2}, q_{3}, \ldots\right)=\sum_{i=1}^{m}\left(U_{i}^{\mathrm{QM}}-U_{\mathrm{EL}, i}\left(q_{1}, q_{2}, q_{3}, \ldots\right)\right)^{2}+\Lambda g\left(q_{1}, q_{2}, q_{3}, \ldots\right),
$$

where $y\left(q_{1}, q_{2}, \ldots\right)$ and $g\left(q_{1}, q_{2}, \ldots\right)$ are the objective function to be minimized and the charge conservation constraint, respectively; $U_{i}^{\mathrm{QM}}$ and $U_{\mathrm{EL}, i}\left(q_{1}, q_{2}, q_{3} \ldots\right)$ denote the electrostatic potential at the position of atom $i$ calculated from quantum mechanics and classical mechanics, respectively; $\Lambda$ is the Lagrange multiplier; $m$ is the total number of positions at which $U_{i}^{\mathrm{QM}}$ and $U_{\mathrm{EL}, i}$ are evaluated. Normally these positions are chosen in several spherical shells around the molecule, from the surface formed by the van der Waals radius of all the involved atoms to a larger radius $[72,73]$. Variations of this method have been developed by other researchers, for example Henchman and Essex [73]. 
Supramolecule Method The essential part of this method of partial charge parameterization is a weighted fitting, where the target data are also obtained from quantum mechanical calculations [31, 69]. These target data include the interaction energy of the model compound with individual water molecules at their equilibrium positions and the equilibrium separation distance, as well as the dipole moment of the model compound. These target data are collected by approaching individual water molecules from different orientations to each water-accessible atom. As the standard way to determine partial atomic charges in CHARMM potential family [30], the supramolecule method has been welldocumented by Mayne et al. [31].

\subsubsection{Dihedral Potential Parameterization}

Unlike the bond potentials and angle potentials, where any functional form can be used around the energy minimum, dihedral potentials have specific periodic degeneracies and several energy minima and maxima when rotating around a bond. Therefore, a typical parameterization of dihedral interactions aims to reproduce the shape of the energy curve obtained from quantum mechanical calculations [20, 31, 74]. Another common target in the parameterization of dihedral potentials is the rotational energy barrier $V_{n}$ in Equation (2.9) obtained from experiments or quantum mechanical calculations [74].

\subsubsection{Van der Waals Potential Parameterization}

Since the van der Waals interactions together with electrostatic interactions are responsible for many thermal properties, properties such as heats of vaporization, heats of sublimation, heats of adsorption, and solvation energy from quantum mechanical calculations or experimental measurements are widely used as target data in the determination of model parameters. The van der Waals potential parameters often need to be adjusted after the parameterization of partial charges due to coupling 
effects $[68,69]$. Unknown parameters for interacting atom pairs can often be obtained from the parameters for individual atomic species that are known via combination rules. The most popular combination rule performs arithmetic average on minimum energy internuclear distances and geometric average on energy depths [29, 69], given by Equation (2.31)

$$
\begin{array}{r}
r_{m}=\frac{r_{m, i}+r_{m, j}}{2} \\
\epsilon=\sqrt{\epsilon_{i} \times \epsilon_{j}} .
\end{array}
$$

where $r_{m, i}$ and $\epsilon_{i}$ denote the minimum energy internuclear distance and energy depth for the interaction of two atom $i$.

\subsection{Simulation Methods of Infrared Spectra}

\subsubsection{Normal Mode Analysis}

The physical meaning of "normal mode" is that all the atoms in the system vibrate around their equilibrium positions akin to harmonic motion. These normal modes are specific motions of a group of atoms, which help reflect the system geometry as well as the interatomic interactions of the system. Therefore computational predictions of normal modes largely depend on the potentials adopted to describe the interatomic interactions. One major approximation adopted in the implementation of Normal Mode Analysis (NMA) is the harmonic oscillator approximation, where the contribution of the movement along a particular normal coordinate towards the system total potential energy is a quadratic function (see harmonic bond potential in Table 2.1). This approximation, however, is only valid when the motion of the atoms is within a sufficiently small range; in other words, the atomic displacements are required to be

confined within the vicinity of a local energy minimum. As such, any energy contributions of atomic movements that are not captured by a quadratic function will be lost (see Equation (2.3) and Figure 2.5). Normal mode analysis is usually performed 
based on an optimized geometry with respect to both internal and external stresses, where the internal stress is related to interatomic forces related to arrangement of atoms inside the system, and the external stress comes from the limitations imposed by the system size. The essential part of NMA is the evaluation of the Hessian matrix, whose elements are the second derivatives of the total potential energy with respect to each degree of freedom of the simulated system. There are $3 N$ degrees of freedom for a system of $N$ atoms, which can be expressed in terms of the three to six external coordinates (corresponding to translation and rotation) plus internal coordinates such as bond lengths, bond angles, dihedral angles, and improper angles. Once the Hessian matrix has been determined, a secular equation will be solved for the eigenvalues and eigenvectors of the Hessian matrix. The resulting $3 N-3$ eigenvalues are used to calculate the normal mode frequencies of the corresponding vibrational modes, and the eigenvectors are the corresponding normal modes. The fundamentals of NMA have been described elsewhere [75, 76]; nevertheless, a brief walk-through of NMA is given below.

\subsubsection{Frequency and Displacement Vector}

The kinetic energy, $T$, of the system can be expressed as

$$
T=\sum_{i=1}^{N} \frac{1}{2} m_{i}\left[\left(\frac{d x_{i}}{d t}\right)^{2}+\left(\frac{d y_{i}}{d t}\right)^{2}+\left(\frac{d z_{i}}{d t}\right)^{2}\right]
$$

where $N$ is the total number of atoms in the system. If we replace the Cartesian coordinates with mass-weighted coordinates,

$$
q_{1}=\sqrt{m_{1}} x_{1}, \quad q_{2}=\sqrt{m_{1}} y_{1}, \quad q_{3}=\sqrt{m_{1}} z_{1},
$$


and so on $\left(q_{4}=\sqrt{m_{2}} x_{2}\right.$, etc.), then Equation (2.32) becomes

$$
2 T=\sum_{i=1}^{3 N} \dot{q}_{i}^{2}
$$

where $\dot{q}_{i}=d q_{i} / d t$. A second-order Taylor expansion can be used to express the total interatomic potential energy of the system in terms of mass-weighted coordinates:

$$
V=V_{0}+\sum_{i=1}^{3 N}\left(\frac{\partial V}{\partial q_{i}}\right)_{0} \Delta q_{i}+\frac{1}{2} \sum_{i=1}^{3 N} \sum_{j=1}^{3 N}\left(\frac{\partial^{2} V}{\partial q_{i} \partial q_{j}}\right)_{0} \Delta q_{i} \Delta q_{j}+\mathcal{O}\left(q_{i}^{3}\right)
$$

where the subscript 0 denotes the optimized geometry and $V_{0}$ denotes the potential energy of the optimized geometry, which can be arbitrarily set to zero; $\Delta q_{i}$ denotes the increment of the coordinate $q_{i}$. Within the harmonic approximation, the atomic displacement $\Delta q_{i}$ used for the calculation of second derivatives is required to be sufficiently small so that higher-order terms can be neglected. Since this Taylor expansion is performed at an optimized geometry, the first derivative of potential energy with respect to each coordinate is zero. Thus, Equation (2.35) under the harmonic approximation reduces to

$$
V-V_{0}=\frac{1}{2} \sum_{i, j=1}^{3 N} H_{i j} \Delta q_{i} \Delta q_{j}
$$

where the coefficients on the coordinates are Hessian matrix elements,

$$
H_{i j}=\left(\frac{\partial^{2} V}{\partial q_{i} \partial q_{j}}\right)_{0}
$$

The Hessian matrix is a $3 N \times 3 N$ square matrix, with $N$ being the number of atoms in the molecule. Each matrix element is essentially the interatomic force constant of 
the two involved atoms. The Lagrangian of the system is

$$
L=T-V
$$

and Lagrange's equation of motion can be written as

$$
\frac{d}{d t}\left(\frac{\partial L}{\partial \dot{q}_{i}}\right)=\frac{\partial L}{\partial q_{i}} .
$$

Substituting Equations (2.32) and (2.35) into Equation (2.39) and rearranging, one gets the following $3 N$ coupled equations:

$$
\sum_{i=1}^{3 N}\left(\ddot{q}_{i}+\sum_{j=1}^{3 N} H_{i j} \Delta q_{j}\right)=0
$$

As normal modes are essentially collective vibrations of all the atoms in the studied molecule, general solutions of the above linear differential equation set are trigonometric functions and exponentials. One possible solution is

$$
q_{i}=A_{i} \cos \left(\gamma^{\frac{1}{2}} t+\varphi\right) .
$$

Substituting Equation (2.41) into Equation (2.40), we get

$$
\sum_{i=1}^{3 N} \sum_{j=1}^{3 N} A_{j}\left(H_{i j}-\delta_{i j} \gamma\right)=0,
$$

where $\delta_{i j}$ is the Kronecker delta and $A_{i}$ is the vibration's amplitude. Solving the secular equation (2.42), one gets the $3 N$ eigenvalues, $\gamma_{i}$, and $3 N$ eigenvectors. The eigenvalue $\gamma_{i}$ is related to the normal mode frequency $\nu_{i}$ by the equation

$$
\nu_{i}=\frac{\sqrt{\gamma_{i}}}{2 \pi},
$$


and the eigenvectors, also called displacement vectors, describe the displacements of each atom of the corresponding normal mode. These displacement vectors are usually normalized and are often used to help assign normal modes to specific molecular motions via mode visualization. Note that among the $3 N$ normal mode frequencies derived from the above secular equation, three of them are close to zero, corresponding to three translational modes.

\subsubsection{Qualitative Intensity}

Another important observable of normal modes is the intensity of the vibration. Generally speaking, the intensity is proportional to the probability of the transition between two vibrational energy levels; in most cases, between the vibrational ground state and the first excited state. In the case of normal mode analysis in classical mechanics, IR intensity of the $k^{t h}$ normal mode can be calculated from the square of the derivative of molecular dipole moment $\mathbf{M}$ with respect to the $k^{\text {th }}$ normal coordinate as follows [77]:

$$
I_{k} \propto\left|\frac{\partial \mathbf{M}}{\partial Q_{k}}\right|^{2}=\sum_{j=x, y, z}\left(\frac{\partial M_{j}}{\partial Q_{k}}\right)^{2} .
$$

In Equation (2.44), $Q_{k}$ is the $k^{\text {th }}$ element of the normal coordinate vector, defined by Equation (2.45),

$$
\mathbf{Q}=\mathbf{D}^{T} \mathbf{q}
$$

where $\mathrm{D}$ is a $3 N \times 3 N$ square matrix, of which each column is an eigenvector. This

definition of normal coordinate suggests that the normal coordinate of the $k^{\text {th }}$ mode is the scalar product of the corresponding normalized eigenvector and mass-weighted Cartesian coordinate vector

$$
Q_{k}=\sum_{i=1}^{3 N} D_{k, i}^{T} \times q_{i}
$$


It also suggests that one can expand the mass-weighted Cartesian coordinates in terms of all $3 N$ normal coordinates,

$$
q_{i}=\sum_{i=1}^{3 N} D_{i, k} \times Q_{k}
$$

Note that in Equation (2.44), $\partial M_{j} / \partial Q_{k}$ can be expanded with the chain rule as

$$
\frac{\partial M_{j}}{\partial Q_{k}}=\sum_{i=1}^{3 N} \frac{\partial M_{j}}{\partial q_{i}} \frac{\partial q_{i}}{\partial Q_{k}}
$$

In Equation (2.48), the term $\partial M_{j} / \partial q_{i}$ is the contribution of the atomic displacement $q_{i}$ towards the $j$ component of the molecular dipole moment, which is given by

$$
\frac{\partial M_{j}}{\partial q_{i}}=\frac{Z_{i} e}{\sqrt{m_{i}}} \times \delta_{i j}
$$

where $Z_{i}$ is the atomic number, unique for the same atom. The second term can be evaluated to be $D_{i, k}$ according to Equation (2.47). Thus,

$$
\begin{gathered}
\frac{\partial M_{j}}{\partial Q_{k}}=\sum_{i=1}^{3 N} \frac{Z_{i} e}{\sqrt{m_{i}}} \times \delta_{i j} \times D_{i, k}, \\
I_{k} \propto \sum_{j=x, y, z}\left(\sum_{i=1}^{3 N} \frac{Z_{i} e}{\sqrt{m_{i}}} \times \delta_{i j} \times D_{i, k}\right)^{2} .
\end{gathered}
$$

Note that in Equation (2.51), the expression inside the parentheses has a different value for each of the three Cartesian directions; this is because in Equation (2.48), only the atomic displacements along the $j$ direction will survive. Equation (2.51) suggests that with the partial atomic charges of individual atoms and eigenvectors derived from normal mode analysis, one can determine the normal mode intensities. For more detailed information on the derivation of the infrared intensity, see Reference 77. Quantitative calculations use Equation (2.52), given by Thomas et al. [17] 
and Neugebauer et al. [78],

$$
I_{k}=\frac{1}{4 \pi \epsilon_{0}} \frac{N_{A} \pi}{3 c^{2}} \sum_{j=x, y, z}\left(\frac{\partial M_{j}}{\partial Q_{k}}\right) .
$$

where $N_{A}$ is Avogadro's number and $c$ is the speed of light in vacuum.

\subsubsection{Molecular Dynamics}

Molecular dynamics (MD) method was first applied by Alder and Wainwright [79] in fluid dynamics, and later became a more valuable simulation tool in many areas of chemistry and physics due to the development of computers and models. Two families of molecular dynamics methods have been developed: quantum molecular dynamics and classical molecular dynamics. Most quantum mechanical MD, also called $a b$ initio MD, is based on the adiabatic approximation, which can be applied due to the difference in time scale of motion of the electrons compared to the nuclei. In this case, the procedure of calculating the next position of each atom is broken into two steps; the first step neglects the nuclear kinetic energy, and the electronic energy is solved self-consistently as a function of the positions of nuclei, which gives rise to a potential energy. This potential energy, together with the kinetic energy of nuclei, forms the Hamiltonian of the system. In the second step, the total energy of the system and forces acting on the atoms are time-integrated numerically. Classical MD obtains the system potential energy via interatomic potentials, which are supposed to reproduce the quantum mechanical potential energy as much as possible without the need for an explicit-electron calculation. The use of the interatomic potentials largely reduces the time consumed for molecular dynamics simulations, making long-time simulation of large systems such as biological molecules and crystals possible. Since classical MD is adopted in the present work, some basic aspects of classical MD simulations are briefly discussed below. 


\subsubsection{Integration Algorithm}

The main idea of classical molecular dynamics is solving Newton's equations of motion numerically, so that particle positions and velocities, as functions of time, can be obtained. These data are processed using statistical methods to obtain desired static and dynamic properties of the simulated system. To solve Newton's equations of motion, several integration algorithms have been developed, including the following:

Verlet algorithm [80] This integration algorithm was originally designed for a simulation of the Lennard-Jones fluid. The main idea of the Verlet algorithm is to predict the particle positions based on two former states with a recursive procedure, and to predict the particle velocities from the difference in position between the previous and future time steps.

Velocity Verlet algorithm [81] This integration algorithm was developed on the basis of the Verlet algorithm, and is now the most widely used algorithm. Unlike the Verlet algorithm, particle velocities are updated via the average acceleration within each time step, and particle positions are updated using the particle velocities at the beginning of each time step.

Leap-frog algorithm [82] Explicit particle velocities are calculated in this algorithm, but the particle positions and velocities are updated in an alternative way, apart from each other by half a time step. This feature means that the total energy of the simulated system can not be obtained directly.

The velocity Verlet algorithm is used in the present work, as it is the default integrator in both LAMMPS [28] and GULP [29].

\subsubsection{Initial Structure}

Besides determination via experimental measurements, such as X-ray powder diffraction, an initial guess of the minimum energy configuration of the simulated 
Table 2.6. Basic simulation parameters in MD simulations.

\begin{tabular}{ll}
\hline Simulation parameter & Physical meaning \\
\hline Time step & Time increment used in the integration of Newton's equa- \\
& tions of motion \\
Sampling frequency & Samples acquired per unit time \\
Integrator & Method used to integrate the Newton's equations of motion \\
Simulation length & Total simulation time \\
\hline
\end{tabular}

system can also be obtained from molecular mechanics geometry optimization. Most

of the widely used simulation packages such as GULP [29] have output routines to dump the final optimized geometry, together with other atomic information, which make the preparation of MD input data very convenient. In the case of lager systems such as polymers, or crystalline materials such as zeolites, the initial structure can also be obtained from small asymmetric units with the help of some auxiliary tools. Note that although the structure of the simulated system deforms at each time step, an MD simulation starting with an initial structure far from a local minimum energy configuration is more likely to generate unreasonable results than one starting near a local minimum energy configuration due to the magnitude of the force far from a local minimum.

\subsubsection{Simulation Parameter Set}

"Simulation parameters" in this content refers to the specifications that describe how an MD simulation is to be carried out; this is different from model parameters, which describe the forces and energies between atoms in the simulated system. Table 2.6 lists some basic MD simulation parameters.

\subsubsection{Initial Velocity}

Commonly used initial velocity distributions are the uniform distribution and the Maxwell-Boltzmann (Gaussian) distribution. In both cases, the initial atomic 
velocities are typically chosen to be consistent with an initial temperature according to the relationship between the average kinetic energy and temperature from statistical thermodynamics given by Equation (2.53)

$$
\frac{1}{2}\left\langle m v^{2}\right\rangle=\frac{3}{2} k_{B} T_{\text {initial }}
$$

The sequence of random numbers is generated based on a seed, which determines the start of the sequence. The total linear and angular momentum are typically set to zero during the initialization of the velocities in order to avoid net motion or rotation of the simulated system.

\subsubsection{System Equilibration}

Given a simulated system without external forces acting on, it is the total energy, instead of the temperature of the system, that is conserved during the simulation. Thus, to ensure the system reaches a desired temperature, temperature control is necessary. Another reason to control the temperature is to offset the steady state energy drift due to the accumulation of numerical errors during MD simulations. Several methods have been developed for temperature control in MD simulations, including the following three types of thermostat. The present work uses the NoséHoover thermostat.

Velocity scaling The most intuitive way to control the temperature is to scale the particle velocities directly, since the temperature of a group of particles is related to the particles' average kinetic energy. Variations on this type of thermostat are implemented in some MD simulation packages such as LAMMPS [28]; Instead of strictly scaling the instantaneous temperature to the desired temperature, a fraction by which the instantaneous temperature is scaled to the desired temperature can be introduced into the mechanism, if desired. 
Berendsen thermostat An example of a simple thermostat is the Berendsen thermostat, where a exponential decay, given by Equation (2.54), of the instantaneous temperature toward the desired temperature at each time step is applied.

$$
\frac{d T(t)}{d t}=\frac{1}{\tau}\left(T^{*}-T(t)\right)
$$

One can tell that if the time constant $\tau$ is too large, only a small change of the instantaneous temperature could be achieved during each time step, meaning the Berendsen thermostat will converge very slowly. On the other hand, a time constant $\tau$ which is too small will lead to an unstable system temperature. Note that the Berendsen thermostat reduces to direct velocity scaling if the time constant $\tau$ is equal to the time step used during the MD simulation (see Reference 83).

Nosé-Hoover thermostat The main idea of this thermostat is to introduce an additional degree of freedom, $s$, as an external thermal bath to the physical system being studied in order to create dynamics that converge to the Canonical ensemble at long times. The resulting Hamiltonian of the extended system is given by

$$
\mathcal{H}=\sum_{i}^{N} \frac{\mathbf{p}_{\mathbf{i}}{ }^{2}}{2 m_{i} s^{2}}+V(\mathbf{q})+\frac{\mathbf{p}_{\mathbf{s}}{ }^{2}}{2 \mathcal{Q}}+h k_{B} T^{*} \ln s,
$$

where $\mathcal{H}$ is the Hamiltonian of the extended system; $\mathbf{p}_{\mathbf{i}}$ is the conjugate momentum of physical particle $i ; \mathbf{p}_{\mathbf{s}}$ and $\mathcal{Q}$ are the conjugate momentum and "mass" of the additional degree of freedom, $s ; k_{b}$ is Boltzmann's constant; and $h$ is the number of degrees of freedom of the physical system (see Reference 84, 85).

\subsubsection{Data Processing}

Since most of the properties of interest cannot be obtained from MD simulations directly, statistical methods are widely used in MD simulations. Evaluations of some 
properties of the system are typically associated with ensemble averages, which can be done with the help of the ergodic hypothesis. The ergodic hypothesis states that, given a long enough simulation time, a system will go through all the possible microscopic states consistent with constraints imposed on it by the surroundings (e.g., energy, volume, and number of molecules). Therefore, the time average of a quantity is equal to its ensemble average in the long-time limit, given by Equation (2.56)

$$
\langle A\rangle=\frac{\iint A(t) \delta(\mathcal{H}-E) \mathbf{d} \mathbf{d p d q}}{\iint \delta(\mathcal{H}-E) \mathbf{d p d q}}=\lim _{t_{\mathrm{tot}} \rightarrow \infty} \frac{1}{t_{\mathrm{tot}}} \int_{0}^{t_{\mathrm{tot}}} A(t) d t,
$$

where $A(t)$ is the instantaneous value of the studied quantity; $E$ is the total energy of the simulated system; $t_{\text {tot }}$ is the total simulation length; and the symbol \langle\rangle denotes the ensemble average. Time correlation functions derived from linear response theory [86] are also widely used in the analysis of MD data. Linear response theory states that for any external perturbation $\zeta$ coupled with system property $B$, the effect to the studied system can be expressed as the change of the Hamiltonian, $\mathcal{H}$, of the system from the unperturbed state $\mathcal{H}_{0}$ via Equation (2.57)

$$
\mathcal{H}=\mathcal{H}_{0}-\zeta B(\Gamma)
$$

where $\mathcal{H}$ and $\mathcal{H}_{0}$ denote the Hamiltonian with and without the external perturbation, respectively, and $\Gamma$ denotes one single point in the phase space of $6 N$ dimensions. The ensemble average of the property $A$ of the studied system can be written as

$$
\langle A\rangle=\langle A\rangle_{0}+\langle\Delta A\rangle=\frac{\int A \exp \left(-\beta\left(\mathcal{H}_{0}-\zeta B\right)\right) d \Gamma}{\int \exp \left(-\beta\left(\mathcal{H}_{0}-\zeta B\right)\right) d \Gamma} .
$$

Equation (2.58) describes the static response of the system to the external perturbation, where the perturbation is a constant. A more general case would be applying a time-dependent external perturbation switched off at time $t=0$, so that Equa- 
tion (2.58) would be a function of time $t$. Assuming that the unperturbed property $\langle A\rangle_{0}$ is 0 , the response of the system, $\langle\Delta A(t)\rangle$, decays to 0 as time goes on. In the limit as $\zeta$ approaches zero, we can approximate $\exp (\beta \zeta B)$ in Equation (2.58) with a first-order Taylor approximation, $\exp (\beta \zeta B) \approx 1+\beta \zeta B$; Equation (2.58) then becomes

$$
\langle\Delta A(t)\rangle=\beta \zeta \frac{\int B A(t) \exp \left(-\beta \mathcal{H}_{0}\right) d \Gamma}{\int \exp \left(-\beta \mathcal{H}_{0}\right) d \Gamma} \approx \beta \zeta\langle B(0) A(t)\rangle .
$$

Assuming the ergodic hypothesis, we can replace the ensemble average with the corresponding time average, which gives

$$
\langle\Delta A(t)\rangle=\lim _{t_{\mathrm{tot}} \rightarrow \infty} \beta \zeta \frac{1}{t_{\mathrm{tot}}-t} \int_{0}^{t_{\mathrm{tot}}-t} B\left(t_{0}\right) A\left(t_{0}+t\right) d t_{0}
$$

In Equation (2.60), $t_{0}$ denotes the time origin, which is the integration variable, and the integration runs over all time origins. $B\left(t_{0}\right) A\left(t_{0}+t\right)$ on the right hand side is actually the time correlation function of the two properties, $B$ and $A$. In the case where the two properties are the same, for example, $A\left(t_{0}\right) A\left(t_{0}+t\right)$ is called the autocorrelation function of property $A$. Physically, the time-domain function in Equation (2.60) can be interpreted as the decay of the system response to the perturbation at time $t$. Fourier transforming the time-domain function to the frequency domain, one can decompose the response fluctuation into frequency components. One good example, which is also massively used in the present work, is the calculation of infrared spectra via the dipole-dipole autocorrelation function given by

$$
\langle\mathbf{M}(0) \cdot \mathbf{M}(t)\rangle=\frac{1}{t_{\mathrm{tot}}-t} \int_{0}^{t_{\mathrm{tot}}-t} \mathbf{M}\left(t_{0}\right) \cdot \mathbf{M}\left(t_{0}+t\right) d t_{0}
$$

The Fourier cosine transform is then used to obtain the IR spectrum,

$$
I(\nu)=2 \int_{0}^{\infty}\langle\mathbf{M}(0) \cdot \mathbf{M}(t)\rangle \cos (2 \pi \nu t) d t
$$


where $\nu$ is the frequency and $I(\nu)$ is the corresponding intensity.

In the thesis, both normal mode analysis and molecular dynamics are adopted. Specifically, molecular dynamics are used to collect the data for the calculation of silica polymorphs' IR spectra, and equilibrium geometries, as well as mechanical properties; normal mode analysis are used to help the peak assignments via normal mode visualizations. 


\section{CHAPTER 3}

\section{EVALUATION OF PUBLISHED POTENTIALS}

As discussed in Chapter 1, computer simulation has been widely adopted to investigate catalytic reactions and adsorption occurring inside zeolites. The basis of these computer simulations is a model describing the interactions between the zeolite framework (host) and reacting species or adsorbates (guest). A good understanding of zeolite framework interactions is also necessary when vibrational spectroscopy is used to detect whether certain reaction intermediates are formed on the surface of the zeolite catalysts, or to distinguish between different adsorbed molecules. By comparing the model-predicted infrared (IR) spectrum with the measured IR spectrum, together with other zeolite framework observables such as geometry and bulk modulus, one can tell whether the adopted potential reproduces zeolite framework interactions correctly. This chapter will focus on testing the performance of two published potentials in terms of predicting IR spectra, geometries, and bulk moduli for five silica polymorphs.

\subsection{Potentials}

\subsubsection{The MZHB Potential}

A potential with low point charges for pure siliceous zeolites was published by Sahoo and Nair [21]. This model was developed in order to reduce the computational expense during hybrid quantum mechanics/molecular mechanics (QM/MM) simulations. As a CHARMM-style potential [30], the Lennard-Jones parameters and partial atomic charges were taken from previous work of Zimmerman, Head-Gordon, and 
Bell [27]. As such, the potential was named the Modified Zimmerman-Head-GordonBell potential, or MZHB. Bond and angle force constants were parameterized via fitting to the faujasite (FAU) experimental bulk modulus. Tests of the potential's performance were based on the prediction of the IR spectra, bond and angle distributions, and lattice parameters for six silica polymorphs. As with most CHARMM-style potentials, the valence interactions of the MZHB potential include simple harmonic bond length and angle bending terms:

$$
\begin{gathered}
V_{\text {bond }}=\sum_{\text {bonds }} \frac{1}{2} k_{R}\left(R-R_{0}\right)^{2}, \\
V_{\text {angle }}=\sum_{\text {angles }} \frac{1}{2} k_{\theta}\left(\theta-\theta_{0}\right)^{2},
\end{gathered}
$$

where $R$ and $\theta$ denote the instantaneous bond length and bond angle, respectively; $R_{0}$ and $\theta_{0}$ denote their equilibrium values, and $k_{R}$ and $k_{\theta}$ are force constants. No torsional or Urey-Bradley terms are used. Non-valence interactions are accounted for with Coulomb interactions and Lennard-Jones interactions, given by Equations (3.3) and (3.4),

$$
\begin{gathered}
V_{\mathrm{EL}}=\sum_{i<j} \frac{1}{4 \pi \epsilon_{0}} \frac{q_{i} q_{j}}{r_{i j}}, \\
V_{\mathrm{vdW}}=\epsilon\left[\left(\frac{r_{m}}{r}\right)^{12}-2\left(\frac{r_{m}}{r}\right)^{6}\right],
\end{gathered}
$$

where $r$ and $\epsilon_{0}$ denote the instantaneous interatomic distance and vacuum permittivity; $q, r_{m}$ and $\epsilon$ are parameters for partial atomic charge, minimum energy internuclear distance, and energy depth, respectively. Arithmetic mean and geometric mean combination rules are adopted for $R_{m}$ and $\epsilon$ with

$$
\begin{gathered}
r_{m}=\frac{r_{m, i}+r_{m, j}}{2}, \\
\epsilon=\sqrt{\epsilon_{i} \epsilon_{j}} .
\end{gathered}
$$


Ewald summation [29] is applied to calculate the electrostatic energy among the fixed point charges with a cutoff of $12 \AA$. The cutoff of Lennard-Jones interactions is also $12 \AA$. Note that the non-valence interactions are not excluded between bonded atom pairs separated by one, two, and three bonds, typically known as 1-2, 1-3, and 1-4 interactions, respectively. These non-valence interactions between neighboring atoms are typically excluded in a CHARMM-style potential, because the interactions between 1-2 and 1-3 atom pairs have already been covered by bond and angle interaction terms, respectively; the interactions between 1-4 atom pairs have been partially covered by torsional interaction terms. One possible reason why the MZHB potential does not have to exclude non-valence interactions between these atom pairs is given in Section 3.5. Table 3.1 lists the MZHB potential's parameters. Specifically, for the Lennard-Jones parameter $r_{m}$, we believe that the MZHB potential's authors intended to use the initial parameters in the CHARMM potential family; however, there was an apparent transposition error, which we will address in Section 3.5.

Table 3.1. MZHB model parameters [21].

\begin{tabular}{ccc}
\hline Harmonic bond potential & $k_{R}\left(\mathrm{eV} / \AA^{2}\right)$ & $R_{0}(\AA)$ \\
Si-O & 23.3000 & 1.620 \\
\cline { 2 - 3 } Harmonic angle potential & $k_{\theta}\left(\mathrm{eV} / \mathrm{rad}^{2}\right)$ & $\theta_{0}\left(^{\circ}\right)$ \\
O-Si-O & 6.8000 & 109.470 \\
Si-O-Si & 2.2200 & 149.800 \\
\cline { 2 - 3 } Lennard-Jones & $\epsilon(\mathrm{eV})$ & $r_{m}(\AA)$ \\
Si & 0.00864 & 2.200 \\
$\mathrm{O}$ & 0.00324 & 1.770 \\
Charge & $q(\mathrm{e})$ & \\
Si & 0.700 & \\
$\mathrm{O}$ & -0.350 & \\
\hline
\end{tabular}




\subsubsection{The Core-Shell Model}

The core-shell model is a type of ion pair potential. It was first introduced by Dick and Overhauser for studying alkali halide crystals [35], and has been widely used in computational studies of ionic materials [22]. As one of the popular ways to include electron polarization effect on the molecular interactions, the main feature of the core-shell model lies in the fact that ions are defined as pairs of nuclear cores and electron shells. Usually, the partial charges of the cores and shells are constrained by charge conservation. In the core-shell model studied in this work [22], oxygen anions are separated into cores and shells, while silicon cations are treated as rigid atoms. The total potential energy, given by Equation (3.7), is a combination of the pair-wise short-range and long-range interactions, the angle interactions and the polarization energies between the cores and shells,

$$
V=V_{\text {short-range }}+V_{\mathrm{EL}}+V_{\text {core-shell }}+V_{\text {angle }} .
$$

Short-range interactions are modeled as Born-Mayer potentials with a cutoff of $10 \AA$ as shown in Equation

$$
V_{\text {short-range }}=\sum_{i, j} A_{i, j} e^{-\frac{r_{i, j}}{\rho_{i, j}}}
$$

Note that the above Equation (3.8) only contains the repulsive force. The reason to exclude the attractive part, as stated by the model's developer, is due to how the model is parameterized. Since this potential is parameterized based on a cluster calculation at the Hartree-Fock level, dispersion contributions are not accounted-for. However, as discussed in Section 2.1.3.2, the attractive force of van der Waals interactions, whether it is described by a Buckingham potential or a Lennard-Jones potential, originates from dispersion forces $[65,66]$. Thus, it is reasonable that only the repulsive term is used in the core-shell model. Note that the short-range interactions are only defined for oxygen shells and silicon atoms, which is consistent 
with the assumption that repulsive forces come from electron repulsion. Long-range interactions are accounted by electrostatic interactions between all the particles with an oxygen core charge of 1.062370 e, oxygen shell charge -3.062370 e, and a silicon charge of 4 e. Electrostatic interactions are computed with Ewald summation with a

cutoff of $10 \AA$, given by Equation (3.3). Note that the summation in Equation (3.3) runs over all particle pairs, excluding core-shell pairs associated with the same oxygen ion, whose interaction energies are accounted by the third term in Equation (3.7), known as the self-polarization energies of core-shell pairs. This self-polarization energy is formulated as a harmonic potential given by Equation (3.9), which runs over all core-shell pairs,

$$
V_{\text {core-shell }}=\sum_{i} k_{s, i} r_{i}^{2} .
$$

Three-body interactions are specified as harmonic angle-bending interactions to give rise to additional structure stiffness. Three-body interactions are also only defined between $\mathrm{O}$ shells and Si atoms, given by Equation (3.10),

$$
V_{\text {three-body }}=\frac{1}{2} \sum_{i, j, k} k_{i j k}\left(\theta_{i j k}-\theta_{0}\right)^{2} .
$$

Potential parameters for the core-shell model are listed in Table 3.2.

\subsection{Method}

The computation of lattice parameters of silica polymorphs involved here was performed with GULP [29]. Initial crystallographic data were obtained from the IZA website [1]. For each calculation, geometry optimization was first performed using molecular mechanics to minimize the total energy with respect to both internal stress and external stress via relaxing atomic positions and the simulation box volume. Model-predicted lattice parameters were then compared with thermallycorrected data obtained via either linear extrapolation or interpolation from exper- 
Table 3.2. Core-shell model potential parameters [22].

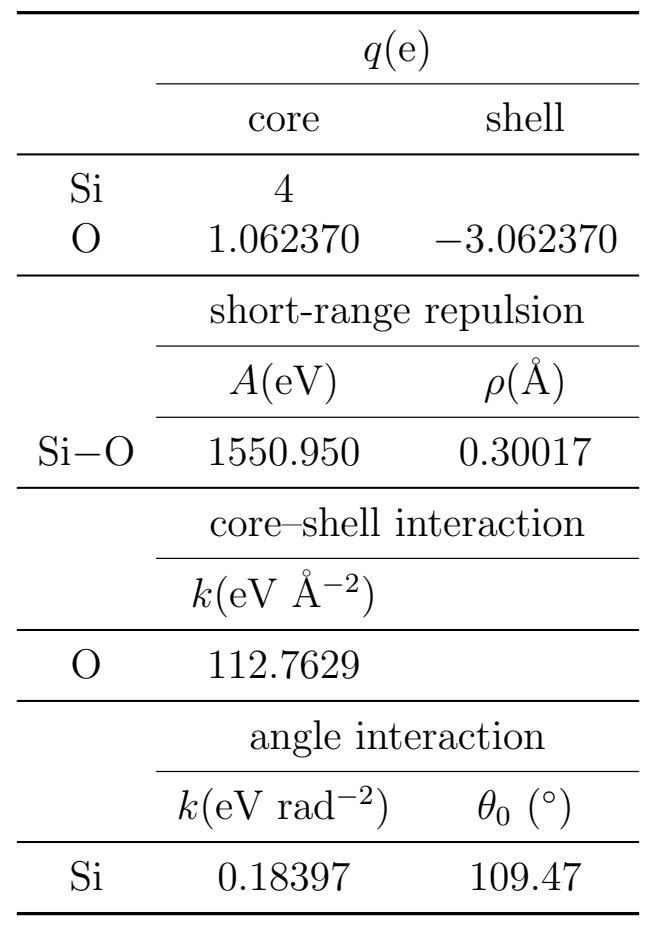

imental data. With the energy-minimized structure, normal mode analyses (NMA) were then performed to calculate the IR spectra. NMA is one of the widely used approaches for the calculation of materials' vibrational modes based on the harmonic approximation. For a system with $N$ atoms, a $3 N \times 3 N$ Hessian matrix consisting of the second derivatives of the total potential energy with respect to each Cartesian degree of freedom, is solved for $3 N$ normal mode vibrational frequencies and atomic displacement vectors. These atomic displacement vectors are related with the intensities of the calculated normal modes. The details of NMA are given in Section 2.2.1.

Molecular dynamics (MD) simulations using LAMMPS [28] were performed to compute the bulk moduli, IR spectra, geometric observables including bond length and angle distributions, and radial distribution functions at $300 \mathrm{~K}$. Bulk moduli were calculated via the change of the average pressure tensors arising from deforming the simulated system along six directions $(x, y, z, x y, x z$, and $y z$; see Reference 28 for 
more information). Bulk moduli were calculated in two different parts of this work via two different ways. In Section 4.1, bulk moduli were calculated via molecular mechanics, and the dependence between the siliceous zeolite's bulk modulus and model parameters was investigated; in Tables 3.4 and 4.2 , bulk moduli were calculated at $300 \mathrm{~K}$ via molecular dynamics. These values are part of the criteria for the evaluation of model performance in this work. Tables of the unique elastic tensor components, and shear moduli from MD simulations are given in Appendix A.

IR spectra were computed with the Fourier cosine transform of the dipole moment autocorrelation function, given by Equation (3.11) and Equation (3.12), respectively,

$$
\begin{gathered}
\langle\mathbf{M}(0) \cdot \mathbf{M}(t)\rangle=\frac{1}{t_{\mathrm{tot}}-t} \int_{0}^{t_{\mathrm{tot}}-t} \mathbf{M}\left(t_{0}\right) \cdot \mathbf{M}\left(t_{0}+t\right) d t_{0} . \\
I(\nu)=2 \int_{0}^{\infty}\langle\mathbf{M}(0) \cdot \mathbf{M}(t)\rangle \cos (2 \pi \nu t)
\end{gathered}
$$

In this work, the autocorrelation function is obtained by post-processing atomic position data with TRAVIS [17, 87, 88].

All the MD-based IR spectra in the present work (see Section 3.3 for how the simulation parameters are determined) are obtained with the following procedure:

1. Obtain the initial configurations from geometry optimization;

2. Run the simulation for 100 ps with a Nosé-Hoover thermostat (fix nvt in LAMMPS) set to $300 \mathrm{~K}$ in place to achieve thermal equilibrium;

3. Run simulations (no thermostat; fix nve in LAMMPS) for 200 ps to produce autocorrelation data [for use in Equation (3.11)];

4. Post-process with TRAVIS.

As mentioned in Section 2.1.3.1, there are two ways to implement the simulation of a core-shell model: the self-consistent method and the extended Lagrangian method. 
In the self-consistent method, positions of massless oxygen shells are optimized via minimizing the total potential energy of the simulated system with no inertial delay at each time step under the adiabatic approximation. No extra degrees of freedom are introduced into the system, however, the computation is much more expensive compared with normal molecular dynamics due to the extra self-consistent steps. The extended Lagrangian method allows shells to have finite masses, and treats shells as particles, that is, Newton's equations of motion are solved for all the particles in the simulated system, including the shells. The extended Lagrangian method is a good approximation to the adiabatic method, when the time scale of the shell movements is negligible compared with that of the core movements; in other words, the frequencies of the change of core-shell pair separation distances are much larger than the frequencies of molecular vibrations [60]. The additional degree of freedom introduces one more simulation parameter, but avoids the time-consuming self-consistent steps. In this work, the extended Lagrangian method is used as implemented in LAMMPS [28].

Infrared peak assignments are performed with the help of a cluster calculation performed on a cluster shown in Figure 3.1a. This cluster consists of a double 6-membered ring (D6R) unit surrounded by four layers of atoms, terminated by $\mathrm{H}$ atoms; this geometry is characteristic of the structure of siliceous FAU. Cluster calculations perform quantum mechanical calculations on small characteristic building units of the entire structure with less expense, circumventing time-consuming and/or intractable quantum mechanical calculations on the entire structure. The properties of these characteristic building units often reflect the properties of the entire structure, to some extent. This is because for some materials in which the electrons are much localized, the central part of the structure will not feel much terminal effects. Thus we can assume that the chemical environment of the central part in a cluster is identical as is in the periodic structure. In the present work, since silicon dioxide is an 


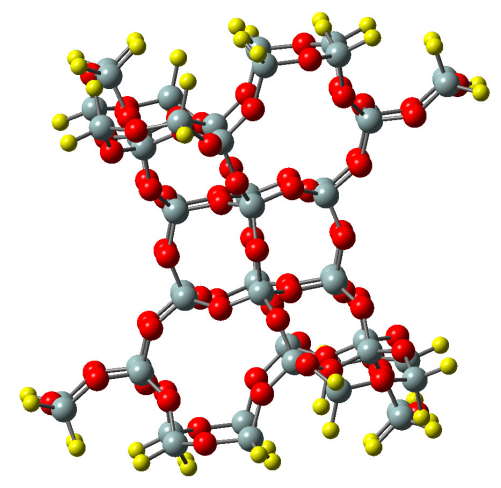

(a) The cluster model used to represent the FAU structure

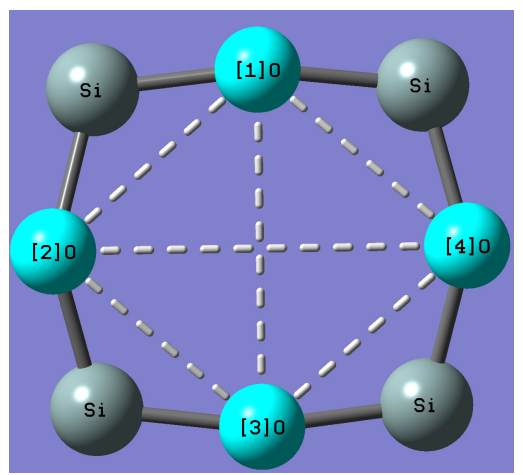

(b) An illustration of the $4 \mathrm{R}$ vibrational analysis

Figure 3.1. Illustrations of the cluster model used for peak assignments in siliceous FAU and the four-ring (4R) vibrational analysis: (a) the cluster model used for peak assignments consisting of a double 6-membered ring (D6R) surrounded by 4 layer of atoms, terminated by $\mathrm{H}$ atoms; (b) an illustration of $4 \mathrm{R}$ vibrational analysis; a quantity, proportional to the area of the $4 \mathrm{R}$, is defined in Equation (3.13).

electron insulator with localized atomic orbitals, cluster calculations can be applied to investigating bulk material properties without introducing significant errors. Fourmembered ring (4R) vibrational frequency analysis is also performed to enhance the peak assignments, as illustrated in Figure 3.1b. In this case, a quantity proportional to the $4 \mathrm{R}$ area is defined by Equation (3.13). Thus, the underlying frequency components of $4 \mathrm{R}$ vibration, that is, the change of the area of the $4 \mathrm{R}$, can be decomposed via Fourier transform of the time autocorrelation function of this quantity [89].

$$
S=\left|O_{1} O_{3}\right| \times\left|O_{2} O_{4}\right|
$$

\subsection{Convergence Study}

Since IR spectra computed from molecular dynamics simulations are based on statistical mechanics, care must be given to the convergence to the ensemble average of 
the model-predicted IR spectra with respect to all the associated simulation parameters. In the original work of the MZHB potential [21], a Nosé-Hoover thermostat [84] with a time constant of 0.1 ps was first applied in an simulation of 50 ps, using a time step of 1 fs, IR spectra were computed from a subsequent 200 ps simulation without the thermostat.

The simulation of the core-shell model in the original work of Schröder and Sauer [22] was performed with molecular mechanics (MM) and normal mode analysis (NMA). Thus, to simulate the core-shell model with MD, a set of reasonable simulation parameters are also needed to be determined. In this work, several test runs were performed first to explore the effects of simulation parameters upon IR spectra and energy conservation during MD simulations. Test simulations investigating the effects of model size, time step, sampling frequency, initial atomic velocities, and simulation length were first carried out using the MZHB potential; test simulations investigating the effect of shell/core mass ratio were then carried out. The optimized simulation parameters based on this convergence study are listed in Table 3.3.

Table 3.3. MD simulation sizes and parameters adopted in this work.

\begin{tabular}{cc}
\hline Structure & Simulation size \\
\hline Cristobalite & $6 \times 6 \times 6(2592$ atoms $)$ \\
Quartz & $6 \times 6 \times 6(1944$ atoms $)$ \\
SOD & $4 \times 4 \times 4(2304$ atoms $)$ \\
FAU & $3 \times 3 \times 2(2592$ atoms $)$ \\
MFI & $2 \times 2 \times 2(2304$ atoms $)$ \\
\hline Parameter & Value \\
\hline Time step & $0.1 \mathrm{fs}$ \\
Sampling frequency & every 100 steps $\left(10^{-14} \mathrm{~s}\right)$ \\
Equilibration time & $100 \mathrm{ps}$ \\
Simulation length & $200 \mathrm{ps}$ \\
Shell/core mass ratio & 0.01 \\
\hline
\end{tabular}

\footnotetext{
${ }^{a}$ The shell/core mass ratio applies only to the core-shell model
} 


\subsubsection{Effects of System Size}

To see whether the size of the simulated system has an effect on the predicted IR spectra, three test MD simulations were performed for quartz with systems of different sizes. The resulted IR spectra are presented in Figure 3.2. Note that the test simulations predict convergent IR peak wavenumbers for quartz with respect to the size for the simulated system, whereas relative peak intensities are slightly affected by the size. No significant effect of the system size on the low-wavenumber region of the calculated spectrum is observed, probably because those vibrational modes in the low-wavenumber region are not IR-active; on the other hand, most of the zeolite framework characteristic vibrational modes are located in the region from $400-1200 \mathrm{~cm}^{-1}$, thus, we think the low-wavenumber vibrational modes will not effect our results significantly. One can tell that, for silica polymorphs, a simulated structure with nearly 3000 atoms is sufficiently large to reduce statistical noise in the IR spectrum. Therefore, system sizes of other types of silica polymorphs are chosen following this observation.

\subsubsection{Effects of the Time Step}

The simulation time step was also tested to find the largest time step that could still avoid energy drift during the solution of Newton's equations of motion. This energy drift comes from inaccuracies in the numerical integration. For example, velocity Verlet integration [81] updates the atomic velocities via the average accelerations within that time step, and updates the atomic positions via the current velocities and current accelerations. This feature of the numerical integration deviates the simulated atomic trajectory from the real moving path which is energy-conserving, thus causing energy errors during each time step.

Besides developing more advanced integrators, one intuitive way to reduce the en-

ergy drift is to decrease the time step used for numerical integration. Figure 3.3 shows 


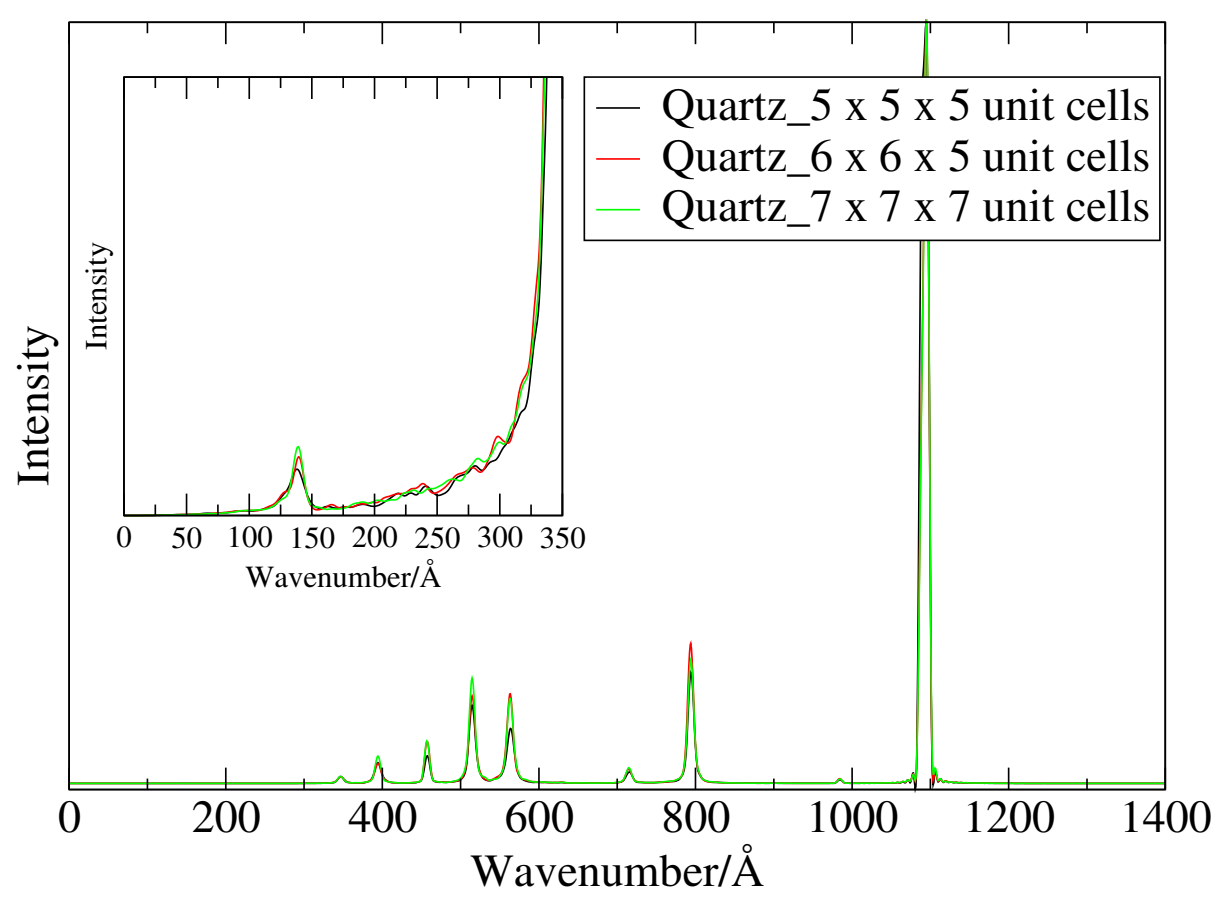

Figure 3.2. MZHB-predicted IR spectra for quartz with different system sizes: simulations were performed for quartz structures with a grid of $5 \times 5 \times 5,6 \times 6 \times 5$, and $7 \times 7 \times 7$ unit cells, corresponding to 1125, 1620, and 3087 atoms, respectively; convergences in peak wavenumbers are achieved, only minor differences in peak relative intensities are observed; the inset shows that the low-wavenumber region of the calculated spectrum is not affected by the system size significantly, probably because the vibrational modes in this region are not IR-active. Simulation parameters used: time step, $0.1 \mathrm{fs}$; sampling frequency, every 5 steps; equilibration time, $100 \mathrm{ps}$; simulation length, $200 \mathrm{ps}$. The same random number seed was used in each case, but the velocities are still different because of the different number of atoms in each one. 


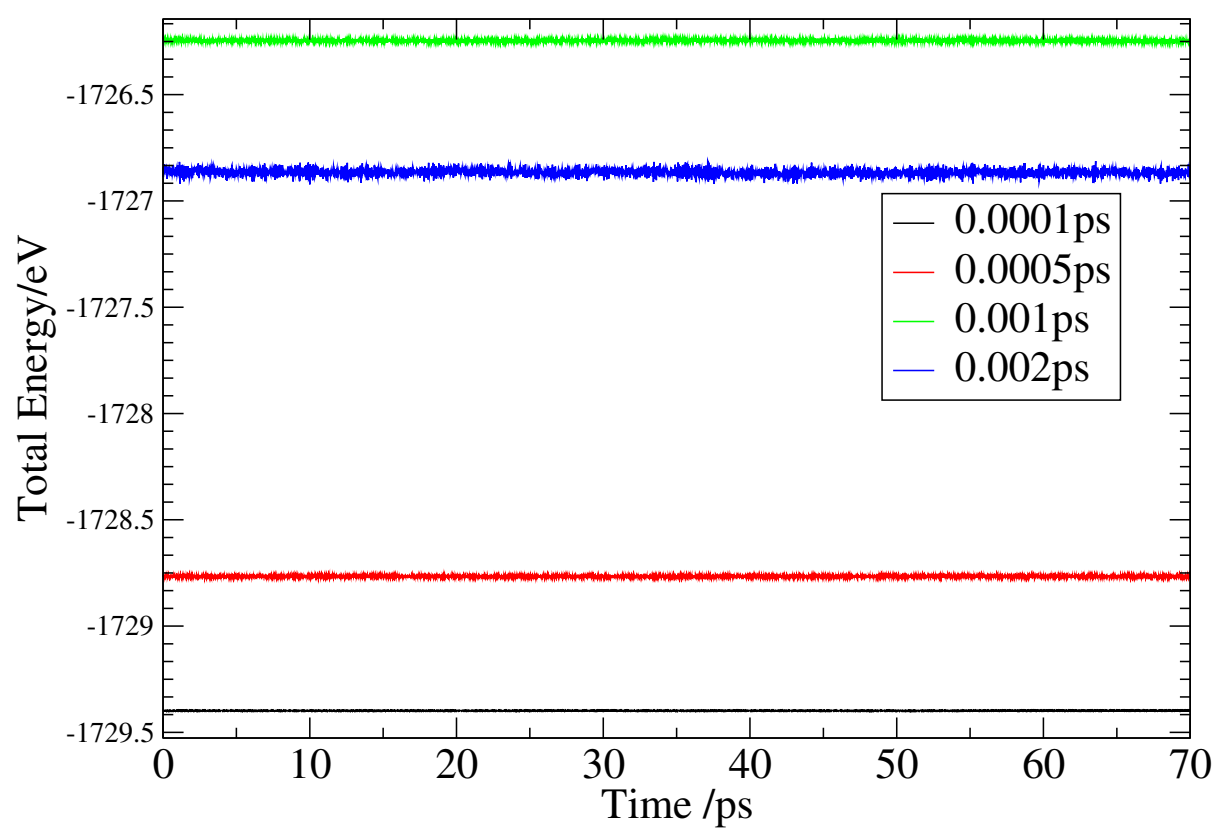

Figure 3.3. The total energy evolution during FAU simulations with different time steps: a smaller time step leads to less energy fluctuations. Simulation parameters used: system size, 1152 atoms; the same random number seed. The differences in the initial total energies originate from the different geometries after a 100 ps equilibration with thermostat.

the total energy evolution of four simulations with different time steps, all sampled during a simulation after 100 ps thermal equilibration, one can see the reduction of noise in the total energy with increasingly smaller time steps; the energy fluctuations from simulations with a time step 2 fs and 1 fs are much larger compared with that from simulations with a time step 0.5 fs and 0.1 fs.

Another possible reason for the energy drift is that possibly some individual atoms are moving in and out of the cutoff distance during the simulation, so that the longrange energy components of the total potential energy vary over time. This is normal and hard to circumvent, since atoms are always moving, and the magnitudes of their displacements vary depending on the specific forces the atoms encountered. Normally, contributions from the cutoff issue are trivial compared to the contributions from unreasonably large time steps. For example, although we observed some atoms moving in and out of the cutoff distance during the simulation using a time step 
$0.1 \mathrm{fs}$, fluctuations of the total energy occurs only at the fourth decimal place (see Figure 3.3). However, for computational studies in which the long-range Coulomb and van der Waals interactions are required at a high accuracy, attention must be paid to find a suitable cutoff that avoids the above issue and/or modify the model to mitigate the energy drift problem, via non-abrupt cutoffs, for example.

Figure 3.4 shows the dependence of the IR spectrum on the choice of the time step. All three simulations were carried out at identical conditions except for the time step and the sampling frequency. These two simulation parameters are coupled in trajectory sampling, for reasons that will be discussed in Section 3.3.3. Here, for each simulation, the time interval between two sampled data is kept at $1 \mathrm{fs}$ in all the three runs. We observe good convergence of the IR peak wavenumbers with respect to the time step: only small differences in peak intensities are observed. Therefore, we believe that a time step of 0.1 fs is sufficiently small for the MZHB potential at $300 \mathrm{~K}$.

\subsubsection{Effects of Sampling Frequency}

The sampling frequency is the other parameter, other than the time step, that determines the time interval between two data points. The sampling time interval in the real time scale is the product of the time step and the sampling frequency, as described by Equation (3.14), where $\Delta t$ and $\Delta t_{s}$ denote the time step and the sampling interval, respectively, and $\nu_{s}$ denotes the sampling frequency.

$$
\Delta t_{s}=\Delta t \times \nu_{s}
$$

According to the Nyquist sampling theorem, the sampling interval in the real time scale $\Delta t_{s}$ should be smaller than at most half of the period of the signal component of the highest frequency. Figure 3.5 shows an illustration of this issue, in which the black $y=\sin (x)$ and red $y=\sin (5 x)$ signals are identical when sampled at a particular 


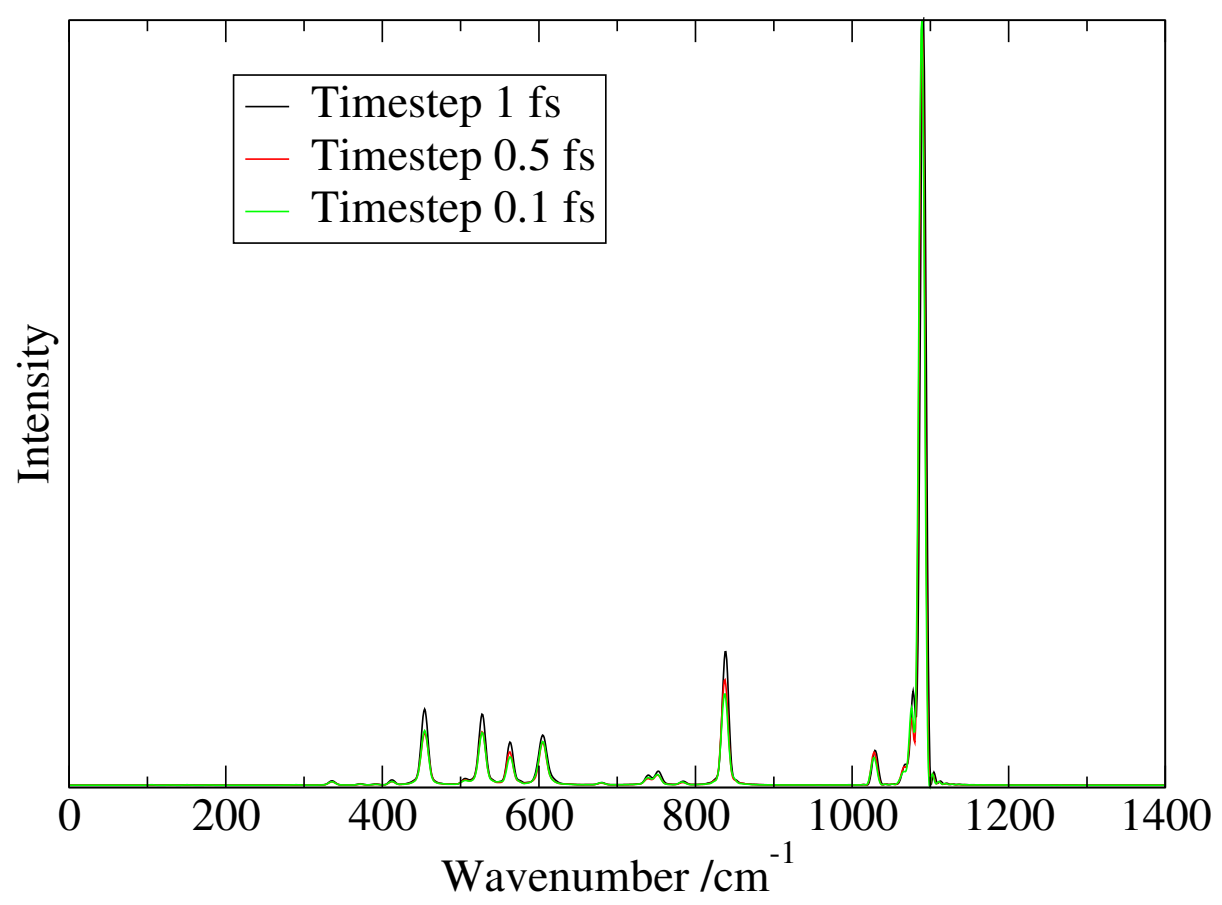

Figure 3.4. FAU IR spectra calculated with three different time steps: good convergence is observed in peak wavenumbers; relative intensities among the peaks differ slightly. Simulation parameters used: system size, 1152 atoms; sampling frequency, every 5 steps; equilibration time, 100 ps; simulation length, 200 ps; the same random number seed.

frequency. While the shape of the black signal is roughly captured, the shape of the red signal is not captured by this sampling frequency. A higher sampling frequency is needed in order to capture red signal's shape better.

Thus, in the case of vibrational spectroscopy, a suitable sampling frequency needs to be found after determining the integration time step, to reduce the computing and storage expenses without sacrificing the maximum-frequency component of the studied molecular vibrations. Figure 3.6 shows the dependence of FAU IR spectra on the sampling frequency, all the simulations are carried out under identical conditions with a time step $0.1 \mathrm{fs}$, except for the sampling frequency. Combining the time step and the sampling frequency together, sampling intervals for the nine runs are $0.4 \mathrm{fs}$, 0.5 fs, 1 fs, 2 fs, 3 fs, 5 fs, 7 fs, 9 fs, and 10 fs, respectively. Perfect convergence of the FAU IR spectrum in terms of both peak locations and relative intensities are 


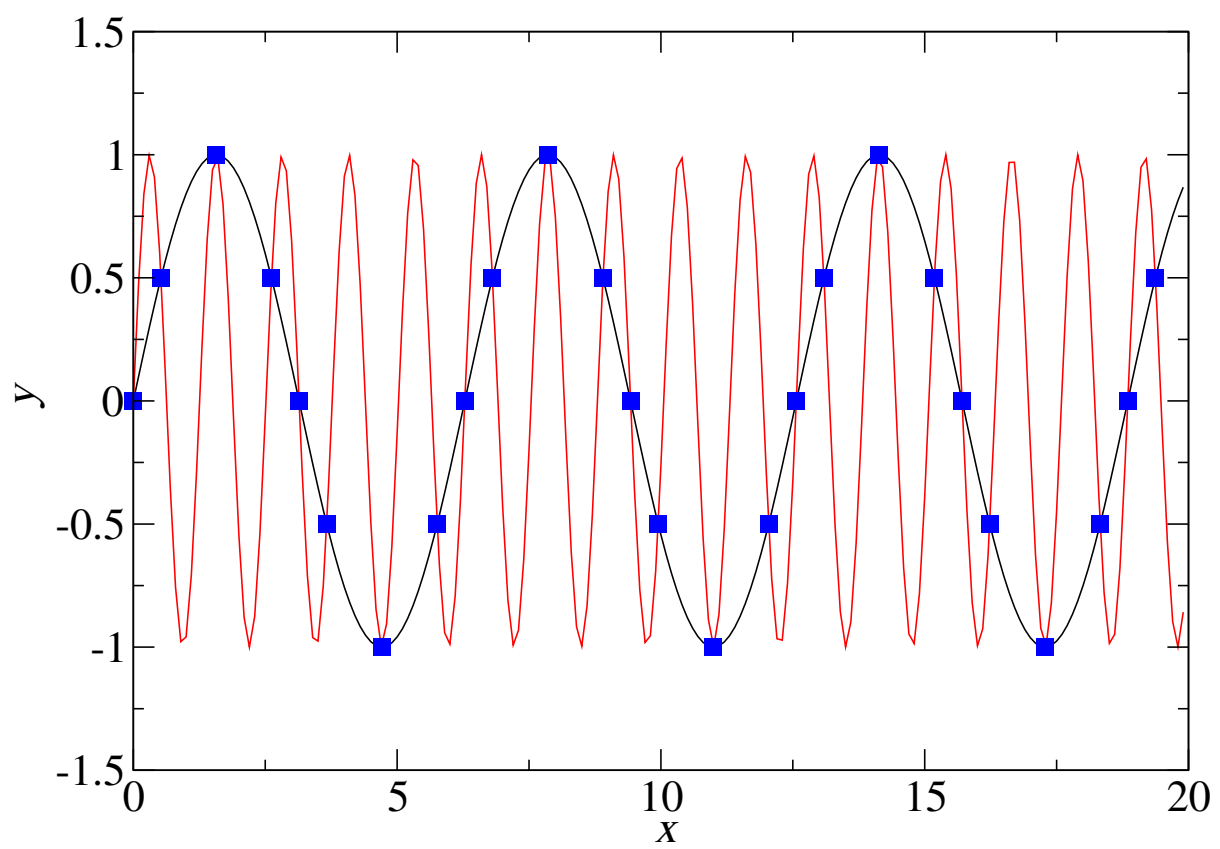

Figure 3.5. An illustration of aliasing in sample acquisition: the black $[y=\sin (x)]$ and red $[y=\sin (5 x)]$ signals are identical when sampled at a particular frequency (blue squares); a higher sampling frequency is needed in order to distinguish these two signals.

observed, and the IR peak of the maximum wavenumber is well reproduced in all the spectra. This convinced us that with a time step of $0.1 \mathrm{fs}$, a sampling frequency of 1/100 time steps $\left(0.1 \mathrm{fs}^{-1}=10^{14} \mathrm{~Hz}\right)$ is sufficiently small, yet guarantees the integrity of the resulting spectrum.

\subsubsection{Effects of the Initial Atomic Velocities and Simulation Length}

Obviously, the trajectories evolved during MD simulations will depend on the initial atomic positions and velocities. However, according to the ergodic hypothesis, given sufficiently long simulation length, each microscopic state in the phase space can be reached with equal probability. This means that a suitable simulation length needs to be found, so that the properties that we observe have no dependence on the initial atomic velocities. Figure 3.7 compares the IR spectra from three simulations of length 200 ps with different initial atomic velocities generated by different 


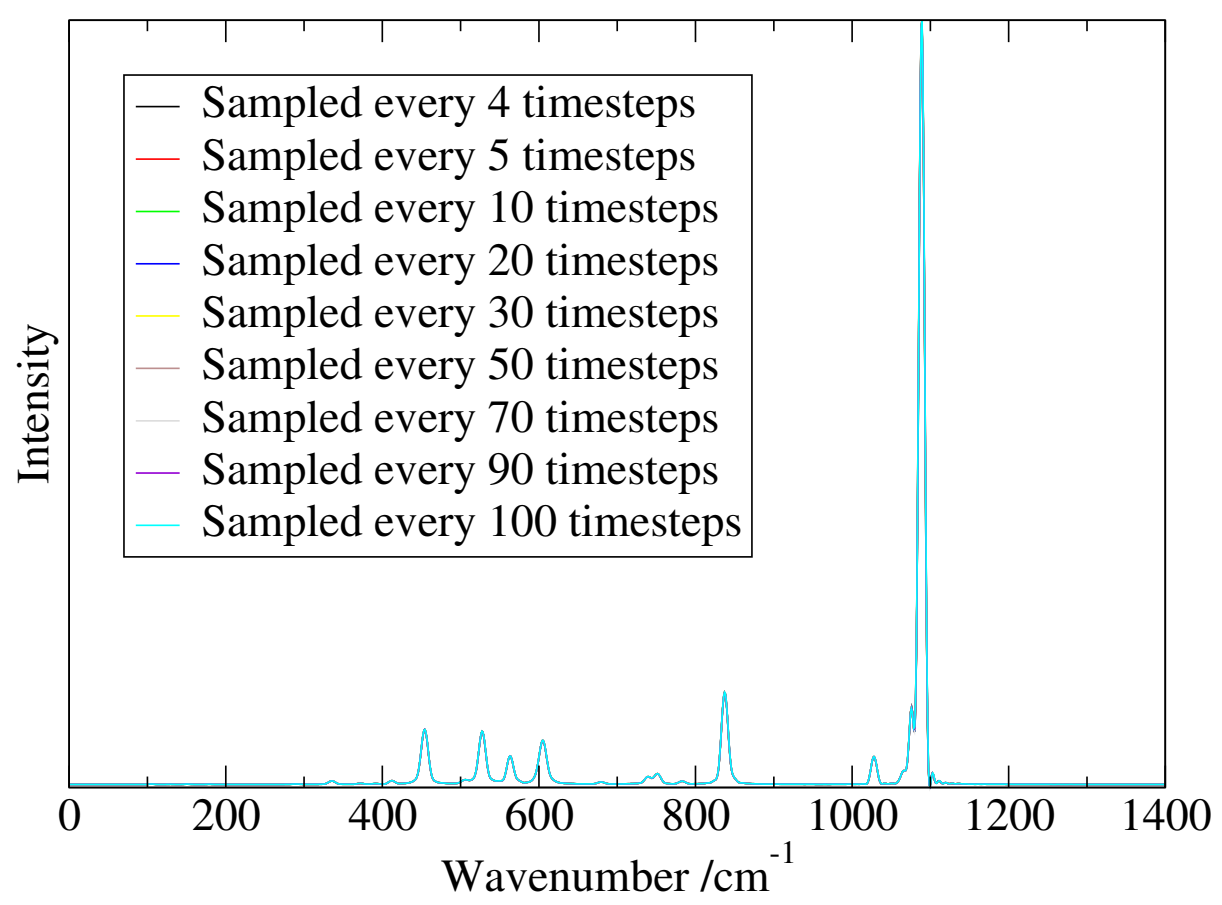

Figure 3.6. FAU IR spectra dependence on the choice of sampling frequency: perfect convergences are observed in both peak wavenumbers and relative intensities. Simulation parameters used: system size, 152 atoms; time step, 0.1 fs; equilibration time, 100 ps; simulation length, 200 ps; the same random number seed.

random number seeds (see Section 2.2.2.4); all the other simulation parameters are kept the same. Note that the IR spectra shown in Figure 3.7 show little variation with respect to the random number seed; only tiny differences in peak intensities are observed. Simulations with longer lengths should converge better, based on the ergodic hypothesis. Statistically, the variance of the intensities of the IR peaks should decrease with the increase of the simulation length. Increasing the simulation length also benefits the resolution of the resulting spectrum. Theoretically, the absolute resolution of a spectrum is defined as the reciprocal of the simulation length, given by Equation (3.15),

$$
\Delta \nu=\frac{1}{t_{\text {tot }}}
$$


which is equivalent to the sampling frequency over the number of sampled data. In cases when the time domain is sampled for a sufficiently long time, good spectral resolution can be expected; while in some cases when the time domain is not sampled for a sufficiently long time, the zero padding method is often adopted to smooth the spectrum resulting from the Fourier transform, though it does not increase the spectral resolution [90]. The Fourier transform resolution is half of the sampling frequency over the number of bins in the frequency domain. The software used in this work (TRAVIS [87]) implements the zero padding method to increase the number of bins with a padding factor of 4, which leads to a Fourier transform resolution given by Equation (3.16),

$$
\Delta \nu=\frac{\nu_{s} / 2}{2^{2} \times N_{\mathrm{FFT}}}
$$

where $N_{\mathrm{FFT}}$ is the number of bins before zero-padding, which is suggested by TRAVIS based on the length of the input data in order to obtain high resolution, yet avoid too much spectral noise. Thus, all the IR spectra presented in Section 3.4 have the same Fourier transform resolution of $0.41 \mathrm{~cm}^{-1}$, since zeolite characteristic vibrational modes occur in different regions of the spectrum (see Table 3.5), and most of the measured wavenumbers are only accurate to integer numbers of $\mathrm{cm}^{-1}$. Figure 3.8 presents a comparison of IR spectra from three simulations that are 30 ps, 200 ps and 500 ps long, respectively, started with the same random number seed. A Fourier transform resolution of $1.02 \mathrm{~cm}^{-1}$ is used for the three calculated IR spectra via adjusting $N_{\mathrm{FFT}}$. While a longer simulation length will ensure better statisticallyaveraged results, the increase of the simulation length from 200 ps to 500 ps does not improve the IR spectral convergence significantly, therefore, we think that $200 \mathrm{ps}$ is sufficiently long for the calculation of silica polymorphs' IR spectra using the MZHB potential. 


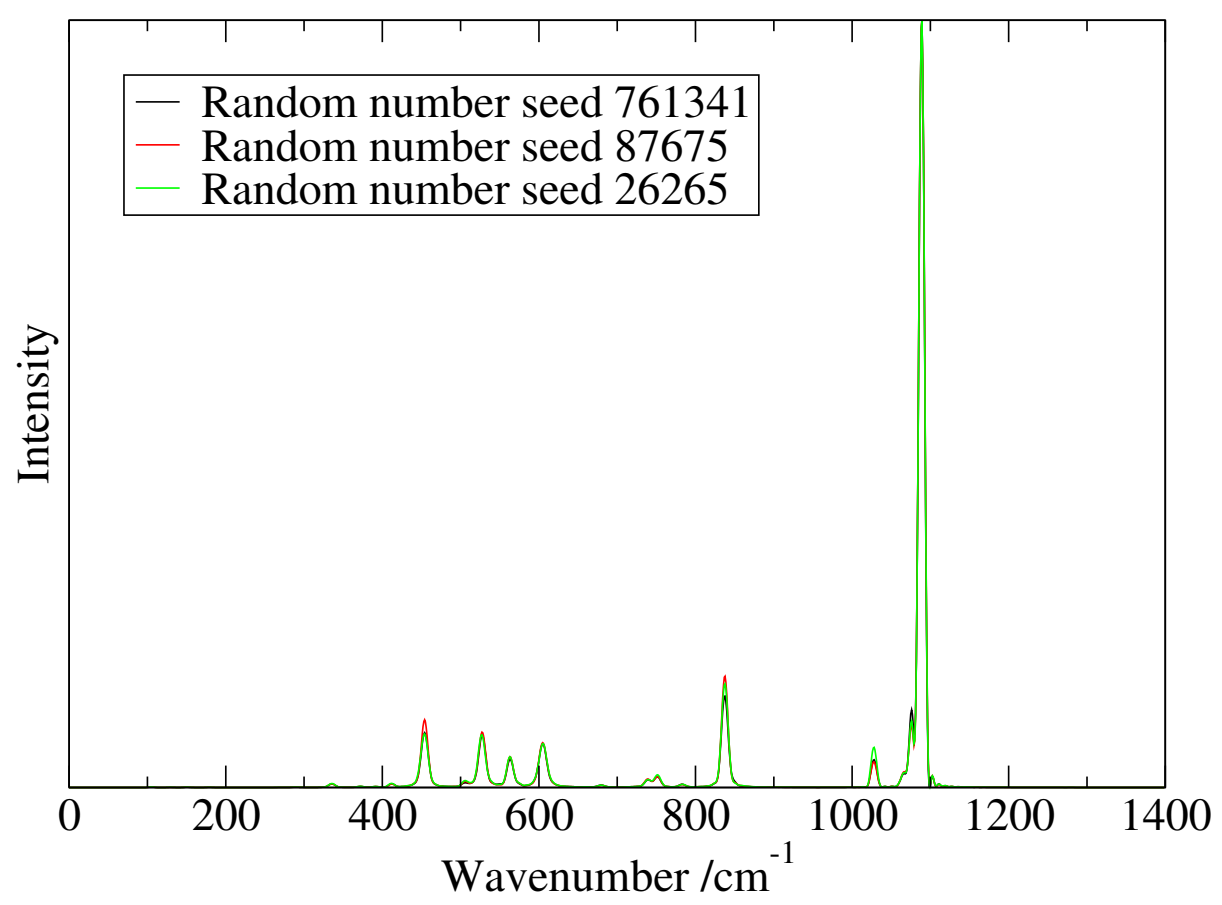

Figure 3.7. FAU IR spectra dependence on the random number seed: Only tiny differences in relative intensities are observed. Simulation parameters used: system size, 1152 atoms; time step, 0.1 fs; sampling frequency, every 5 steps; equilibration time, 100 ps; simulation length, 200 ps.

\subsubsection{Effects of the shell/core mass ratio}

Figure 3.9 shows the evolution of the total energy of three simulations with different shell/core mass ratios and time steps. Obviously, a smaller shell/core mass ratio leads to better total energy conservation, as the energy fluctuation got reduced. However, it is also observed that further reducing of the time step from 0.1 fs to 0.05 fs actually does not improve energy conservation very much, while the computation expenses increase by a factor of two.

\subsection{Results and Discussion}

\subsubsection{Geometric Observables and Bulk Moduli at $300 \mathrm{~K}$}

The fitness of a model for a particular application largely depends on how the model is parameterized, that is, what the target systems are. Since the parameters 


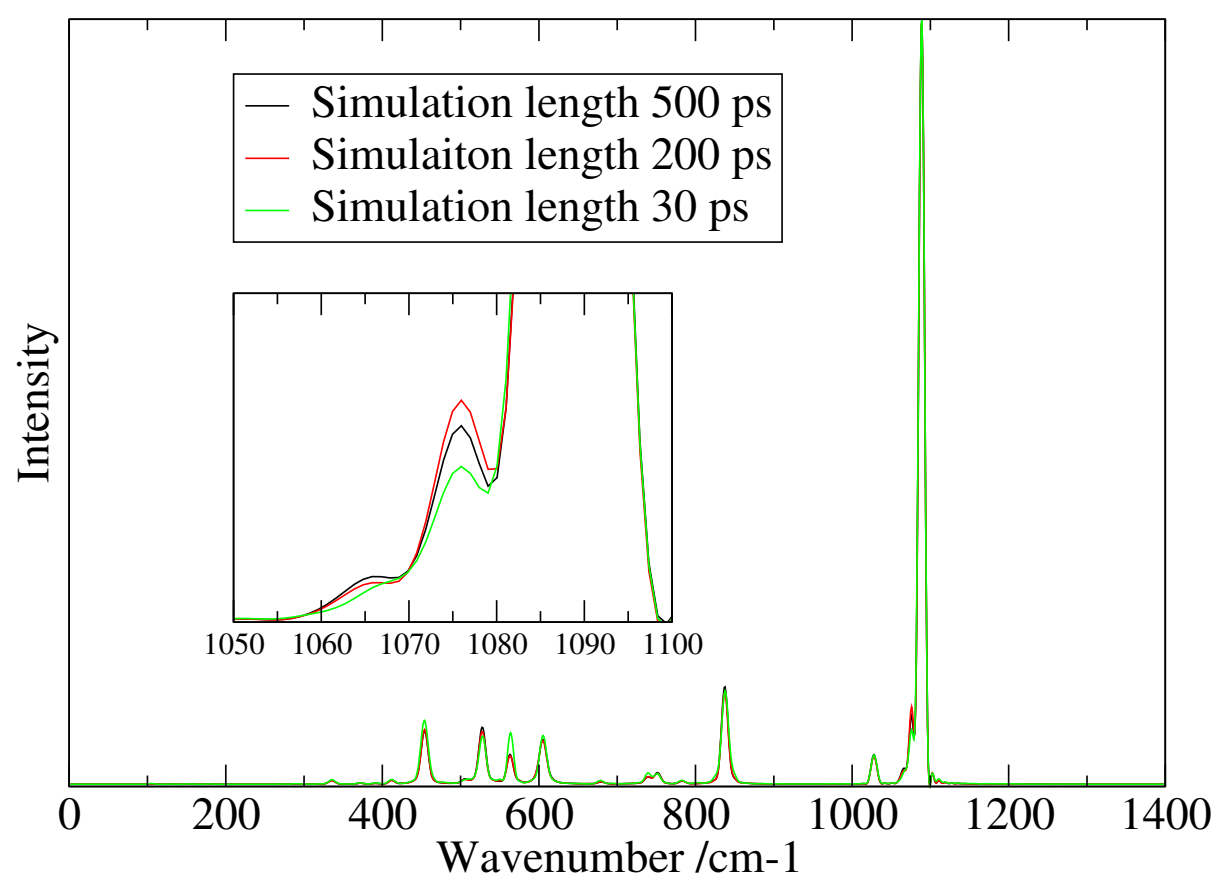

Figure 3.8. FAU IR spectra dependence on the simulation length: only small improvement on spectral convergence has been achieved when the simulation length is increased from 200 ps to 500 ps. Simulation parameters used: system size, 1152 atoms; time step, 0.1 fs; sampling frequency, per 20 steps; equilibration time, $100 \mathrm{ps}$; the same random number seed used. Peak variations shown in the inset are likely originate from statistical errors; differences in resolution (see Equation (3.15)) are not evident in this plot because the affected vibrations (low frequencies) are not IR active.

of the MZHB potential were determined by fitting to the measured FAU bulk modulus, and the core-shell model was parameterized by fitting to geometric observables obtained from quantum mechanical cluster calculations, bulk moduli and equilibrium geometries of silica polymorphs at $300 \mathrm{~K}$ were computed to test the performance of the MZHB potential and the core-shell model.

Calculated geometrical observables and bulk moduli of five silica polymorphs are listed in Table 3.4. Good agreement between model-computed data and experimental data are seen, especially for the average $\mathrm{Si}-\mathrm{O}$ bond lengths and $\mathrm{O}-\mathrm{Si}-\mathrm{O}$ angles. Note that only the computed bulk modulus of FAU is close to the experimental value, indicating poor transferability of the MZHB potential to other silica polymorphs 


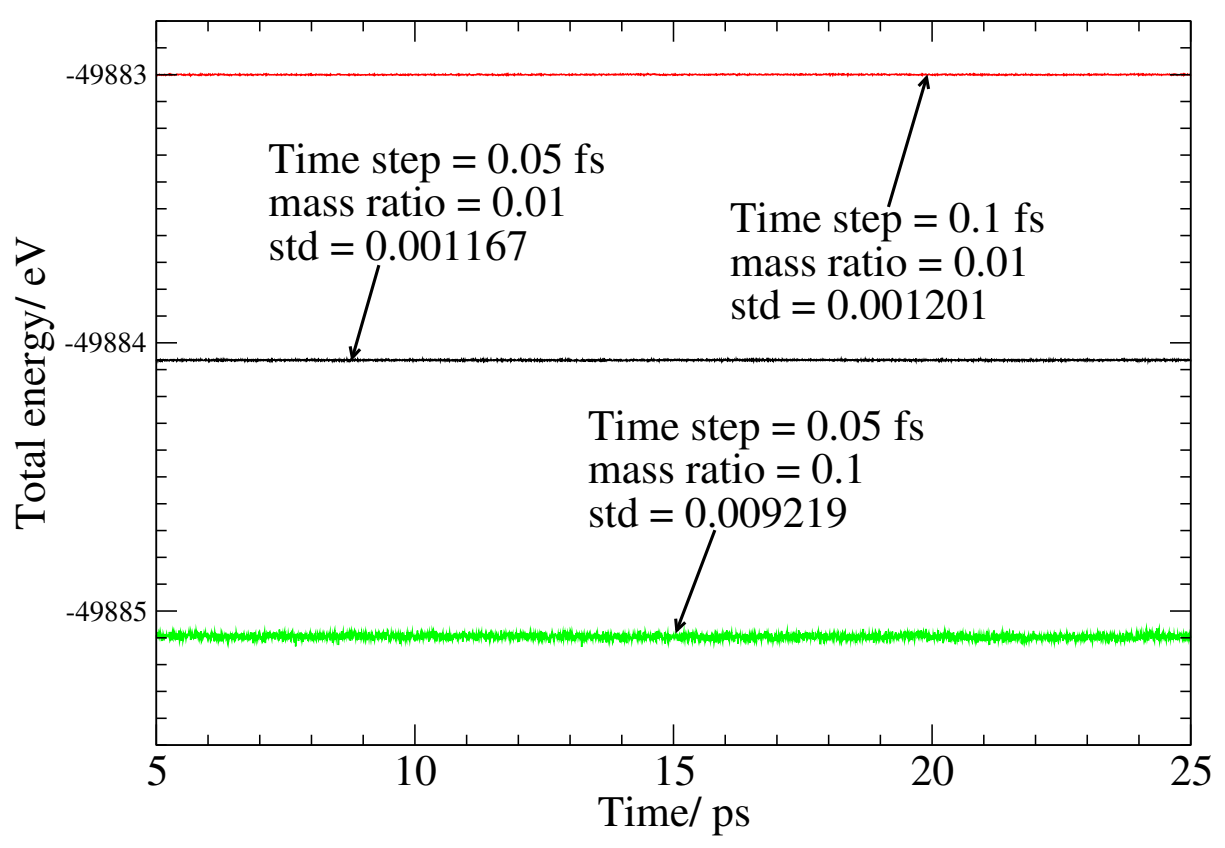

Figure 3.9. The evolution of the total energy of three simulations with varying shell/core mass ratios and time steps: simulated model used, FAU $3 \times 3 \times 2$ (2592 particles); samples are collected during a 20 ps simulation continued from a previous 5 ps equilibration; standard deviations (std) are included in the figure. A smaller shell mass leads to better energy conservation, and with a shell/core mass ratio 0.01, it is not worthwhile to decrease the time step from 0.1 fs to 0.05 fs for better energy conservation.

in terms of the prediction of mechanical properties such as bulk modulus. These discrepancies between model-predicted and measured silica polymorphs' bulk moduli may also originated from the differences between conditions under which experiments and simulations were carried out, such as temperature, pressure, and impurities. For instance, the measured bulk modulus of SOD was based on $\mathrm{Na}_{4} \mathrm{Al}_{3} \mathrm{Si}_{3} \mathrm{O}_{12} \mathrm{Cl}$, while in our simulations, siliceous SOD were used.

Equilibrium bond length and angle distributions as well as radial distribution functions are presented for SOD as examples. These help illustrate how broad typical bond and angle distributions of silica polymorphs can be and how one could study their crystallographic structures via radial distribution functions. 


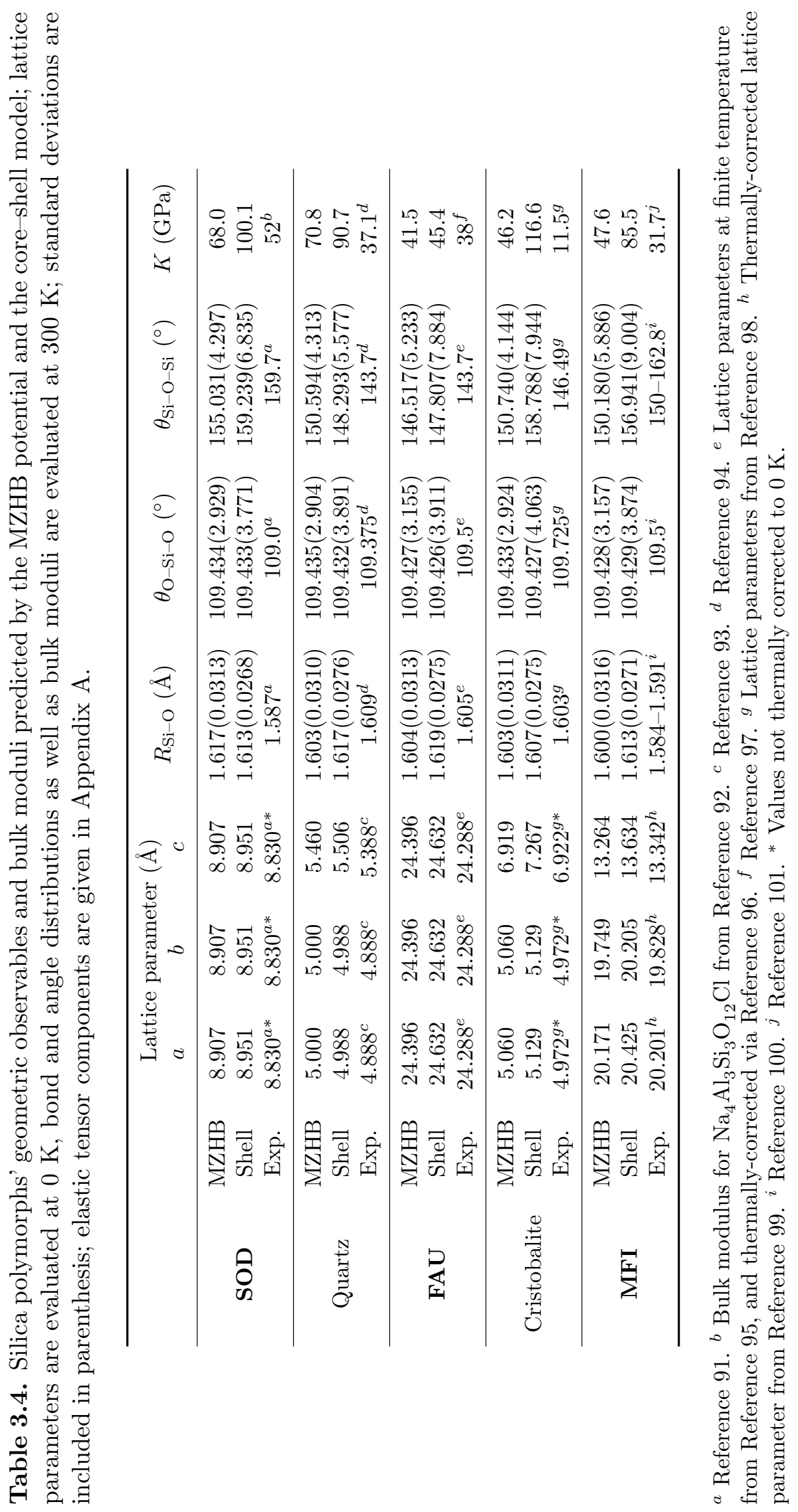




\subsubsection{SOD Geometry}

Figures 3.10a and 3.10b show the SOD $\mathrm{Si}-\mathrm{O}$ bond distribution, $\mathrm{O}-\mathrm{Si}-\mathrm{O}$ and $\mathrm{Si}-\mathrm{O}-\mathrm{Si}$ angle distributions, respectively. The MZHB potential predicts an average bond length $1.617 \AA$ and average angles $109.434^{\circ}$ and $155.031^{\circ}$, respectively; the core-shell model predicts an average bond length $1.613 \AA$ and average angles $109.433^{\circ}$ and $159.239^{\circ}$, respectively. These distributions are well within the range for silica polymorphs' geometric observables [20]. Note that the $\mathrm{Si}-\mathrm{O}$ bond distribution predicted by the MZHB potential is slightly broader than that predicted by the core-shell model, meaning that the interactions between bonded atom pairs are more stronger in the core-shell model than that in the MZHB potential, which gives rise to less broad bond length distribution during the MD simulation. The core-shell model predicts much broader distributions of both the $\mathrm{O}-\mathrm{Si}-\mathrm{O}$ and the $\mathrm{Si}-\mathrm{O}-\mathrm{Si}$ angles, meaning that the three-body interactions in the core-shell model is weaker compared to that in the MZHB potential. The broad Si-O-Si angle distribution predicted by the core-shell model ranges from $140^{\circ}$ to $180^{\circ}$; we think this might be because of its lack of three-body interactions that constrain the deformation of the $\mathrm{Si}-\mathrm{O}-\mathrm{Si}$ angles, which leads to a larger average $\mathrm{Si}-\mathrm{O}-\mathrm{Si}$ angle compared with that predicted by the MZHB potential. This phenomenon due to the lack of three-body interactions was also observed in the work of other researchers [19].

Figure 3.11 depicts the computed radial distribution functions of SOD calculated with both the MZHB potential and the core-shell model. Radial distribution functions reflect the arrangement of atoms in the crystal and indicate the probability of finding atom pairs at specific distances from each other. Mathematically, it is defined as the ratio of the local number density to the overall number density,

$$
g(r)=\frac{\delta n(r)}{4 \pi r^{2} \delta r} / \frac{N}{V}
$$




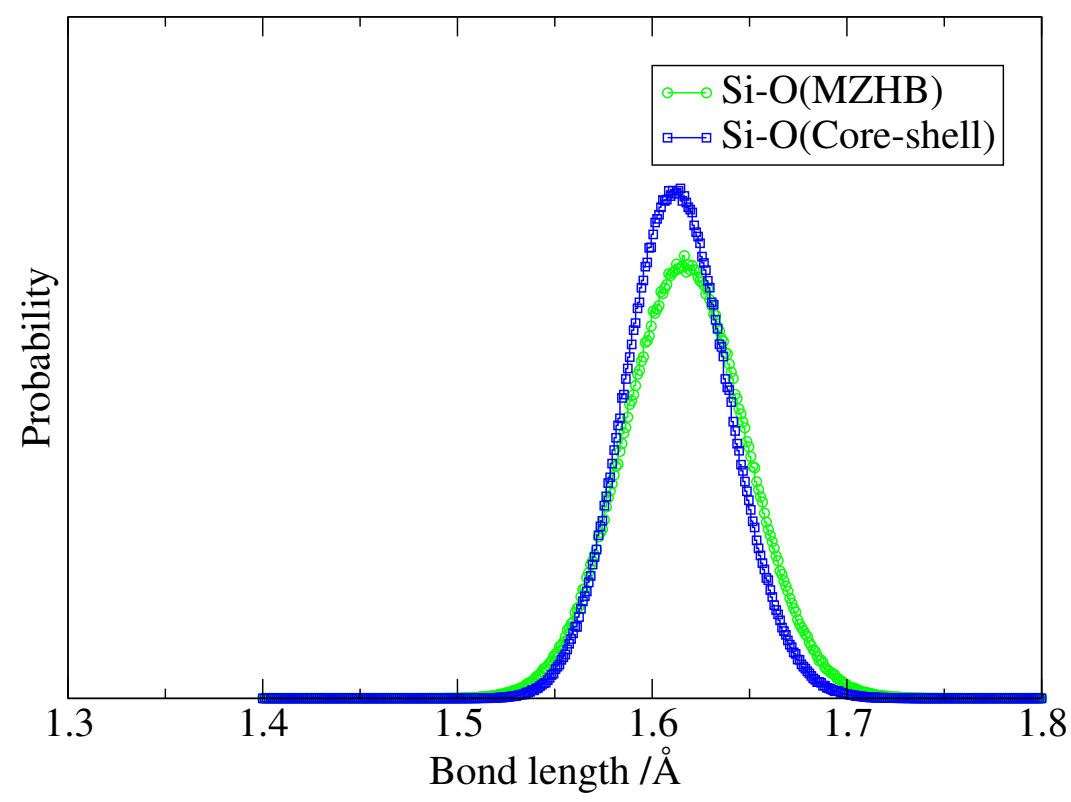

(a) The SOD bond length distribution at $300 \mathrm{~K}$.

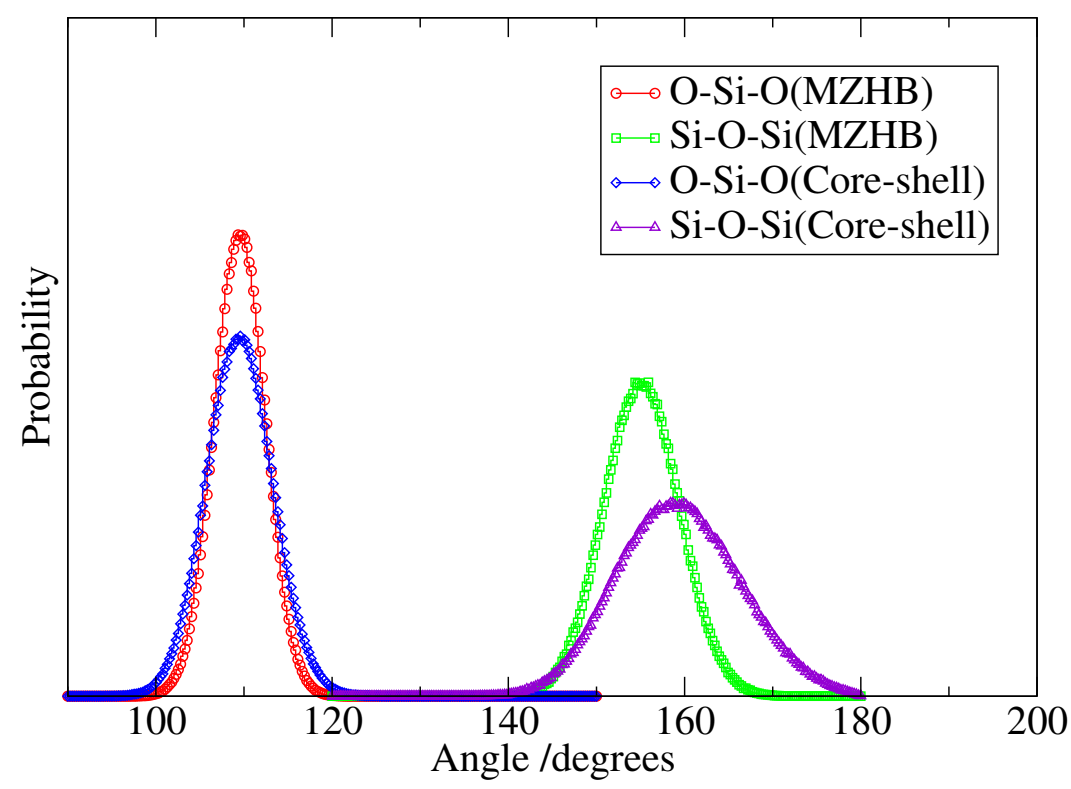

(b) The SOD bond angle distribution at $300 \mathrm{~K}$.

Figure 3.10. The SOD bond length and angle distributions at $300 \mathrm{~K}$ : SOD modeled by the MZHB potential and the core-shell model via molecular dynamics at $300 \mathrm{~K}$ predict average bond lengths of $1.617 \AA$ and $1.613 \AA$, respectively, predict average angle for the $\mathrm{O}-\mathrm{Si}-\mathrm{O}$ angles and the $\mathrm{Si}-\mathrm{O}-\mathrm{Si}$ angles of $109.43^{\circ}$ and $155.031^{\circ}$ (MZHB), $109.43^{\circ}$ and $159.24^{\circ}$ (core-shell), respectively: bond distributions predicted by the core-shell model are observed to be narrower than those predicted by the MZHB potential, meaning that the core-shell model predicts a slightly "stiffer" $\mathrm{Si}-\mathrm{O}$ bond; broader angle distributions are predicted by the core-shell model, probably originating from its lack of three-body interactions to constrain the $\mathrm{Si}-\mathrm{O}-\mathrm{Si}$ angles. 
In Equation (3.17), $\delta r$ and $\delta n$ denote for the radial increment and the number of target atoms within the spherical shell in three dimensional space between $r$ and $r+\delta r ; N$ and $V$ are the total number of atoms and total volume. The radial distribution function eventually decays to 1 as the shell volume increases and the local number density inside the shell approaches to the overall number density.

Obviously, the MZHB potential and the core-shell model predict an almost identical radial arrangement of atoms in the SOD structure, especially in the short range. The sharp peak of the $\mathrm{Si}-\mathrm{O}$ radial distribution function at approximately $1.61 \AA$ confirms the average $\mathrm{Si}-\mathrm{O}$ bond length. The sharp peak of $\mathrm{O}-\mathrm{O}$ radial distribution function indicates a most closest $\mathrm{O}-\mathrm{O}$ distance of about $2.61 \AA$, which has already been confirmed by other researchers and widely implemented in zeolite potential energy models in the formulation of the Urey-Bradley potential $[19,20,33]$. The distance of closest $\mathrm{Si}-\mathrm{Si}$ neighbors of about $3.15 \AA$ is also consistent with other research [20]. Note that the $\mathrm{Si}-\mathrm{Si}$ radial distribution function predicted by the core-shell model is at slightly higher separation distances in all the observed peaks; this is consistent with the broad $\mathrm{Si}-\mathrm{O}-\mathrm{Si}$ angle distribution in Figure 3.10b, in the sense that a larger average $\mathrm{Si}-\mathrm{O}-\mathrm{Si}$ angle gives rise to a larger $\mathrm{Si}-\mathrm{Si}$ separation distance, given the fact that the average $\mathrm{Si}-\mathrm{O}$ bond lengths predicted by both two models are almost identical. The difference of $\mathrm{Si}-\mathrm{O}$ radial distribution function at around $4 \AA$ is also consistent with the above argument.

\subsubsection{Infrared Spectra}

Zeolite measured IR spectra peak assignments have been studied by Flanigen et al. [102]; a typical wavenumber range of IR peaks associated with zeolite framework vibrations lies between $400 \mathrm{~cm}^{-1}$ and $1200 \mathrm{~cm}^{-1}$. While peaks from the vibrations of local $\mathrm{SiO}_{4}$ tetrahedra are assigned approximately to the same wavenumbers among various types of zeolites, the wavenumbers of external linkage peaks vary accord- 


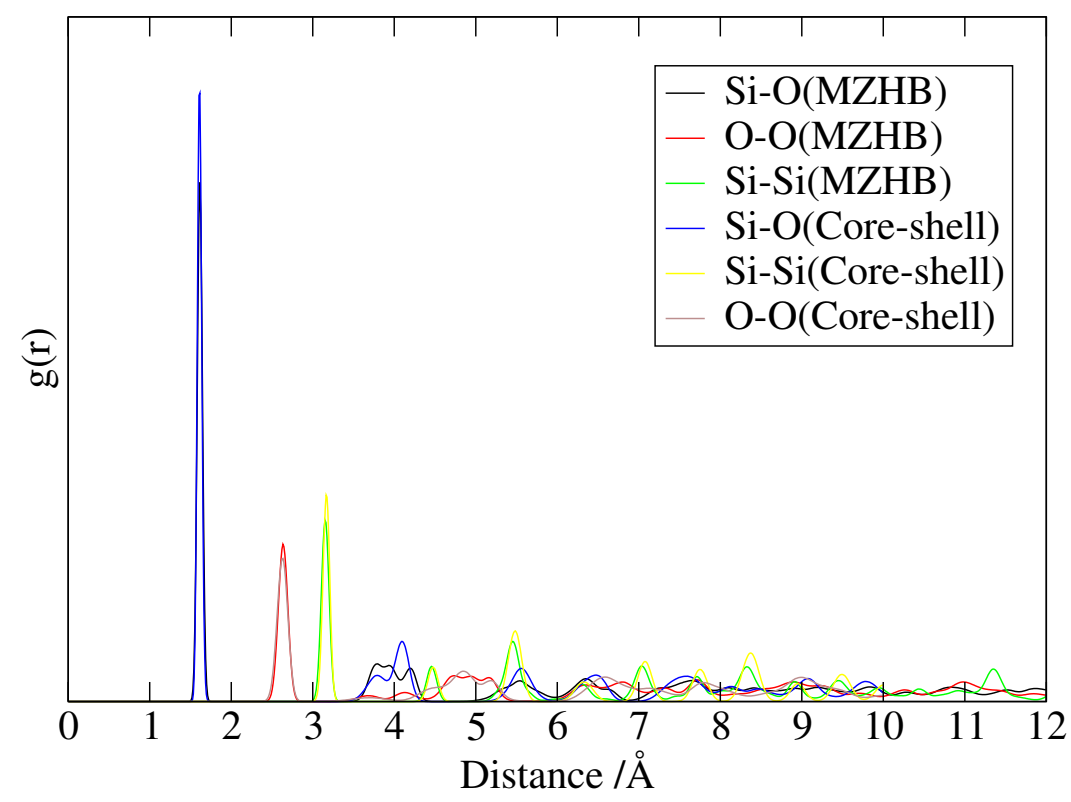

Figure 3.11. SOD radial distribution functions at $300 \mathrm{~K}$ : predictions from both models are very similar; the distance between the closest neighbor of $\mathrm{Si}-\mathrm{O}, \mathrm{O}-\mathrm{O}$, and $\mathrm{Si}-\mathrm{Si}$ are found to be about $1.61 \AA, 2.61 \AA$ and $3.15 \AA$, respectively.

ing to the specific zeolite framework. This is because internal tetrahedral peaks are structure-insensitive, while external linkage peaks are structure-sensitive [102]. Typical zeolite IR peak assignments [102] are listed in Table 3.5. Structure-insensitive peaks, such as the asymmetric bond-stretching peak in the $950-1250 \mathrm{~cm}^{-1}$ range and the angle-bending peak in the $420-500 \mathrm{~cm}^{-1}$ range, are observed in different zeolites [103].

Testing the performance of a potential is often linked to potential applications of that model. Since the performance of the MZHB potential was checked by comparing the model-predicted FAU IR spectrum with the measured spectrum, IR spectra at $300 \mathrm{~K}$ are logical observables to test whether the MZHB potential has good transferability to different silica polymorphs. Therefore, MD simulations were performed for the calculation of IR spectra for all five silica polymorphs studied in this work (see Table 3.3). Infrared spectra computed via NMA are also presented. Normalmode analysis can not only help probe the anharmonicities of the adopted potential 
Table 3.5. Zeolite IR peak assignments made by Flanigen et al. [102].

\begin{tabular}{lcc}
\hline & Vibrational motion & Wavenumber Range $\left(\mathrm{cm}^{-1}\right)$ \\
\hline \multirow{2}{*}{$\begin{array}{l}\text { Internal } \\
\text { tetrahedra }\end{array}$} & Asymmetric stretch $v_{a s}(\mathrm{O}-\mathrm{Si}-\mathrm{O})$ & $950-1250$ \\
& Symmetric stretch $v_{a}(\mathrm{O}-\mathrm{Si}-\mathrm{O})$ & $650-720$ \\
& Angle bending $\delta(\mathrm{O}-\mathrm{Si}-\mathrm{O}) / \delta(\mathrm{Si}-\mathrm{O}-\mathrm{Si})$ & $420-500$ \\
\cline { 2 - 3 } External & Double ring & $500-650$ \\
linkages & Pore opening & $300-420$ \\
& Symmetric stretch $v_{s}(\mathrm{Si}-\mathrm{O}-\mathrm{Si})$ & $750-820$ \\
& Asymmetric stretch $v_{a s}(\mathrm{Si}-\mathrm{O}-\mathrm{Si})$ & $1050-1150\left(\mathrm{sh}^{*}\right)$ \\
\hline
\end{tabular}

* Weak shoulder

by comparing its results to MD-based IR spectra, but more importantly, it helps to match computed IR peaks with associated molecular motions via normal mode visualizations. Note that the normal mode visualization itself is not always easy to be used to confirm peak assignments due to the complexity of the structure of silica polymorphs, as most normal modes have bond-stretching motions and angle-bending motions coupled together. In order to investigate the underlying bond length and angle coupling, we plot the percent change of bond lengths and angles for observed modes with high IR intensities to quantify the coupling effect. Cluster calculations are also performed on the cluster, shown in Figure 3.1a via quantum mechanics to assist in IR peak assignments.

\subsubsection{FAU (siliceous faujasite)}

As stated in the original work of Sahoo and Nair [21], most of the IR peaks in the measured spectrum of siliceous FAU [104] were well-reproduced by the MZHB potential, especially the internal asymmetric stretching $v_{\text {as }}(\mathrm{O}-\mathrm{Si}-\mathrm{O}$ mode at around $1090 \mathrm{~cm}^{-1}$ and the external symmetric stretching $v_{\mathrm{s}}\left(\mathrm{Si}-\mathrm{O}-\mathrm{Si}\right.$ mode at around $837 \mathrm{~cm}^{-1}$. Mismatches between the computed IR spectrum and the measured IR spectrum occur at around $400 \mathrm{~cm}^{-1}, 561 \mathrm{~cm}^{-1}, 790 \mathrm{~cm}^{-1}$, and $1215 \mathrm{~cm}^{-1}$. Among these mismatches, the IR peak at around $400 \mathrm{~cm}^{-1}$ is usually assigned to the pore-opening 
mode $[21,104,105]$. Sahoo and Nair speculated that the two mismatches at around $790 \mathrm{~cm}^{-1}$ and $1215 \mathrm{~cm}^{-1}$ might have originated from aluminum, since enhancement of the intensities of these two peaks with increasing aluminum concentration is observed experimentally [104]. They assigned the mismatch at around $561 \mathrm{~cm}^{-1}$ to the ring block vibration.

In order to see whether the computed IR spectrum predicted by the MZHB potential matches correctly with the measured spectrum, we computed the IR spectrum of siliceous FAU via both NMA and MD with both the MZHB potential and the core-shell model and performed normal mode visualizations to assist in peak assignments. To help reconcile computed IR peaks with measured IR peaks, we performed a quantum mechanical calculation on a cluster (see Figure 3.1a) characteristic of the structure of siliceous FAU using GAUSSIAN [106]. We find (see Appendix B) that this cluster is large enough to reproduce characteristic vibrational modes of the periodic FAU structure.

The cluster calculation was performed at $\mathrm{HF} / 6-31 \mathrm{G}^{*}$; the reason we chose this level of theory and basis set for the cluster calculations was to be consistent with prior studies - many classical mechanical potential are parameterized in this level [31]. Also, HF /6-31G* was observed to be one of the best theoretical levels that minimizes a target function when fitting computed IR frequencies with measured frequencies using scaling factors [107]. The calculated IR spectra are scaled by a factor of 0.8953 [107] (see Appendix B for calculation details). The computed IR spectrum for the cluster is presented in Figure 3.12; peak assignments are based on normal mode visualizations with GaussView [108]. The most important point here is that the experimentally observed major peak in the angle-bending region at around $462 \mathrm{~cm}^{-1}$ is assigned to the $6 \mathrm{R}$ deformation, and two IR modes associated with the $4 \mathrm{R}$ breathing motion are observed on both sides of this major peak. 


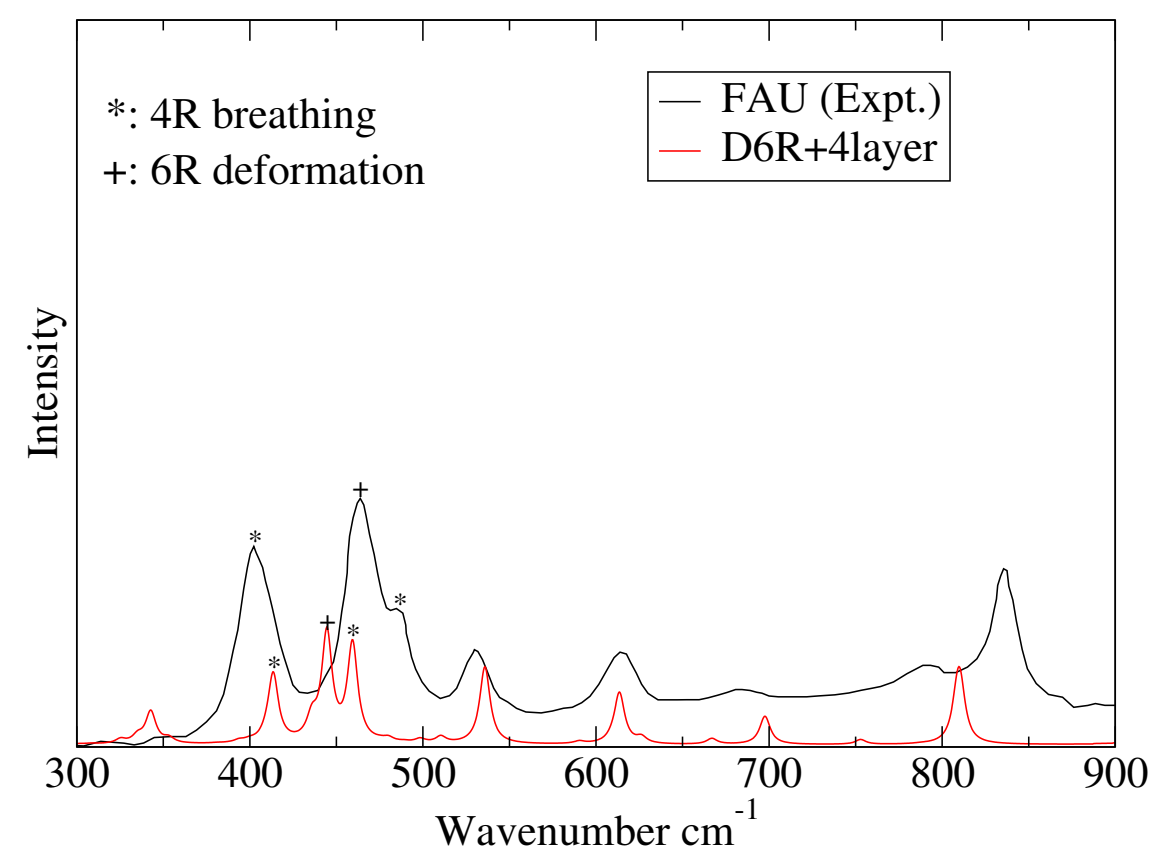

Figure 3.12. IR spectra of the cluster model from the cluster calculation. The experimentally observed major peak in the angle-bending region at around $462 \mathrm{~cm}^{-1}$ is assigned to the six-ring (6R) deformation, and two IR modes associated with the $4 \mathrm{R}$ breathing motion are observed in both sides of this major peak. The measured spectrum was obtained from Jacobs et al. [104].

With the help of the cluster calculation, comparison of the MZHB-predicted IR spectrum and the measured IR spectrum for FAU is presented in Figure 3.13. Peak assignments of the peaks in the angle-bending region are labeled in the figure. These peak assignments are carried out by comparing the underlying molecular motions of the MZHB-predicted IR modes with that predicted by the cluster calculation. In order to support these peak assignments, visualizations of the angle-bending IR peaks, predicted by both the MZHB potential and the cluster calculation, which we believed should match with each other, are presented parallel. All these visualizations focus on the double-six-ring (D6R) unit.

Figure 3.14a presents the underlying molecular motion associated with the IR peak at around $453 \mathrm{~cm}^{-1}$ in the MZHB-computed IR spectrum. Apparently, as is indicated by the arrows, this molecular motion is the $4 \mathrm{R}$ breathing motion originating 


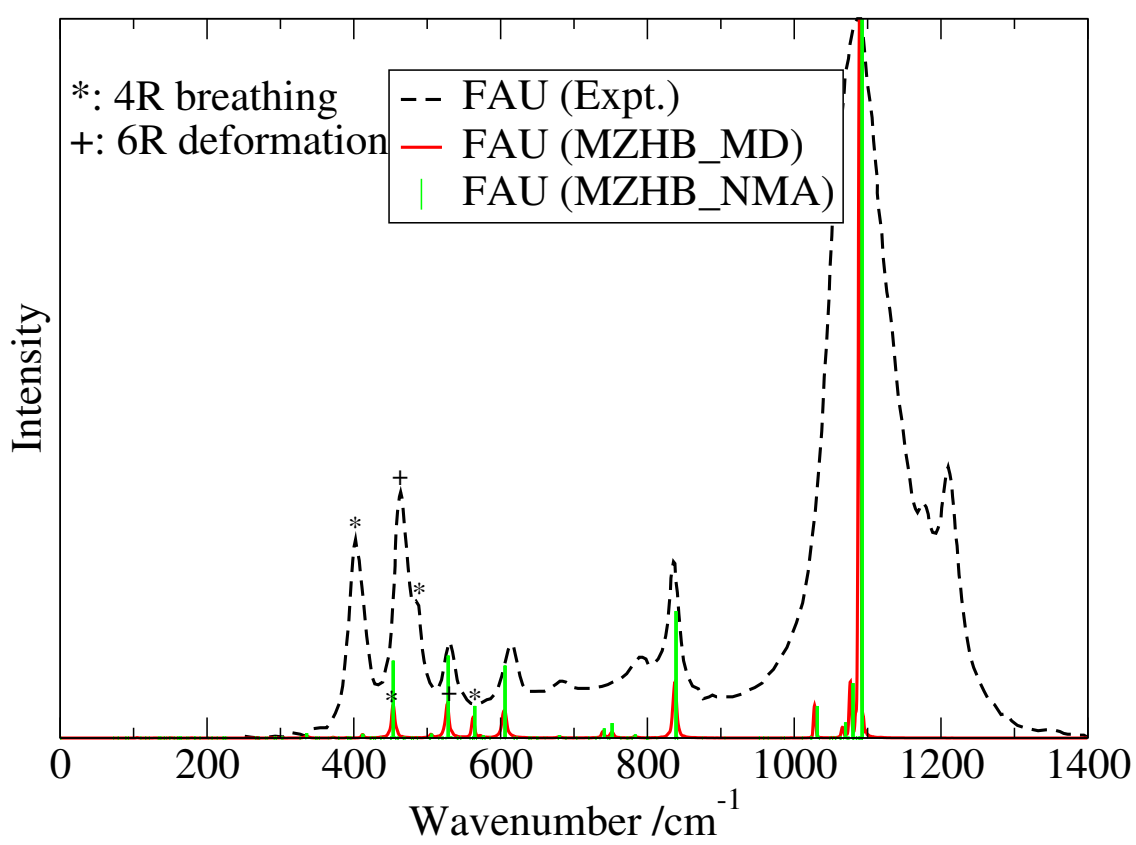

Figure 3.13. FAU IR spectra predicted by the MZHB potential at $300 \mathrm{~K}$ : apparent peak shift to higher wavenumbers are observed in the angle-bending region, indicating that the angle-bending force constants are too large; peak assignments are performed by comparing corresponding underlying molecular motions. The measured IR spectrum is obtained from Jacobs et al. [104].

mainly from the movements of oxygen atoms, occurring on the sides of the D6R unit, where three of the four oxygen atoms in each $4 \mathrm{R}$ move, causing expansion or contraction of the corresponding four-membered ring. While this peak was assigned by the developers of the MZHB potential to the major peak in the angle-bending region at around $462 \mathrm{~cm}^{-1}$ in the measured IR spectrum [21], we think, by comparing to the underlying molecular motion of the cluster IR mode at around $414 \mathrm{~cm}^{-1}$ presented in Figure 3.14b, that this MZHB-predicted peak should be assigned as a $4 \mathrm{R}$ breathing mode; that is, to the pore-opening mode at around $400 \mathrm{~cm}^{-1}$ in the measured IR spectrum.

A further investigation of the visualizations presented in Figure 3.14 shows that these two molecular motions are also characterized by the motion of the six-membered ring (6R). Specifically, three $\mathrm{O}$ atoms of the same 6R, illustrated in Figure 3.15, are 


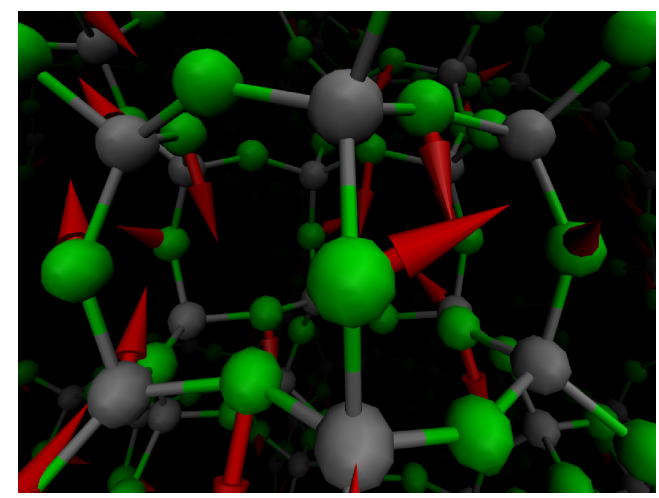

(a) The FAU IR mode at $453 \mathrm{~cm}^{-1}$

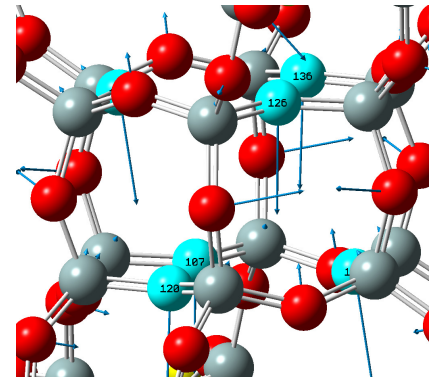

(b) The cluster IR mode at $414 \mathrm{~cm}^{-1}$

Figure 3.14. (a) The FAU IR mode at around $453 \mathrm{~cm}^{-1}$ predicted by the MZHB potential. (b) The cluster IR mode at around $414 \mathrm{~cm}^{-1}$ predicted by the cluster calculation. The underlying molecular motions of these two peaks are both the 4R breathing motion, in both pictures, arrows, which indicate the directions of atomic displacements, show that four-membered rings on the sides of the double six-membered ring unit are either expanding or contracting; the magnitudes of arrows, proportional to the atomic displacements, also indicate that this $4 \mathrm{R}$ breathing motion is mainly originated from the movements of oxygen atoms.

moving up and down around the $6 \mathrm{R}$ plane. Such up-and-down motions are also highlighted in Figure 3.14b.

Note that there is another IR peak at around $460 \mathrm{~cm}^{-1}$ predicted by the cluster calculation, which is assigned to the $4 \mathrm{R}$ breathing motion in Figure 3.12. One would expect that such an IR peak appears on the MZHB-predicted IR spectrum, too. Indeed, another IR peak associated with the $4 \mathrm{R}$ breathing motion is observed in the MZHB-predicted IR spectrum at around $564 \mathrm{~cm}^{-1}$. As is shown in Figure 3.16a, the underlying molecular motion of this mode is also the $4 \mathrm{R}$ breathing motion, which is consistent with the molecular motion of the cluster IR mode predicted at around $460 \mathrm{~cm}^{-1}$, shown in Figure 3.16b. This peak at around $564 \mathrm{~cm}^{-1}$, predicted by the MZHB potential, might be assigned to the measured shoulder at around $482 \mathrm{~cm}^{-1}$, since this peak moves closer to the major peak in this region when increasing the cluster size (see Appendix B). 


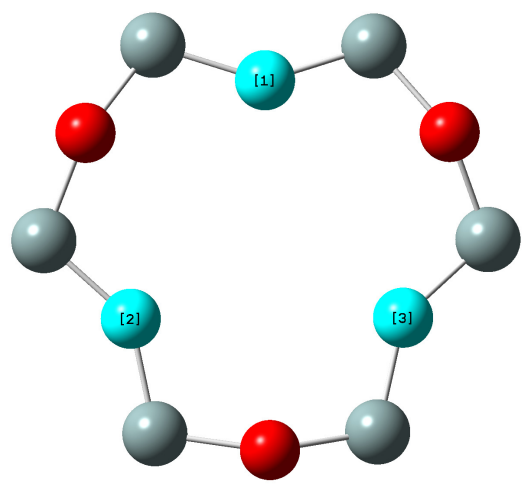

Figure 3.15. An illustration of the motions of oxygen atoms in an attached $6 \mathrm{R}$ associated with the $4 \mathrm{R}$ breathing motion. The three highlighted oxygen atoms move up and down about the $6 \mathrm{R}$ plane due to the breathing motion of the $4 \mathrm{R}$ of which one of the highlighted atoms is also a part.

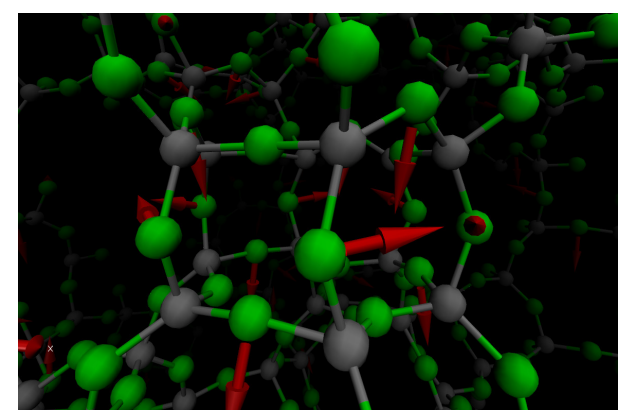

(a) The FAU IR mode at $564 \mathrm{~cm}^{-1}$

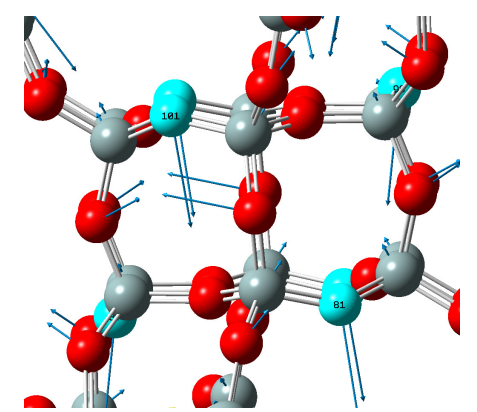

(b) The cluster IR mode at $460 \mathrm{~cm}^{-1}$

Figure 3.16. (a) The FAU IR mode at around $564 \mathrm{~cm}^{-1}$ predicted by the MZHB potential. (b) The cluster IR mode at around $460 \mathrm{~cm}^{-1}$ predicted by the cluster calculation. The underlying molecular motions of these two peaks are also the $4 \mathrm{R}$ breathing motion, in both picture, arrows, which indicate the directions of atomic displacements, show that 4-membered rings on the sides of the double 6-membered ring unit are either expanding or contracting; the magnitudes of arrows, proportional to the atomic displacements, also indicate that this $4 \mathrm{R}$ breathing motion is mainly originated from the movement of oxygen atoms; the highlighted oxygen atoms help illustrate the up-and-down motions. 


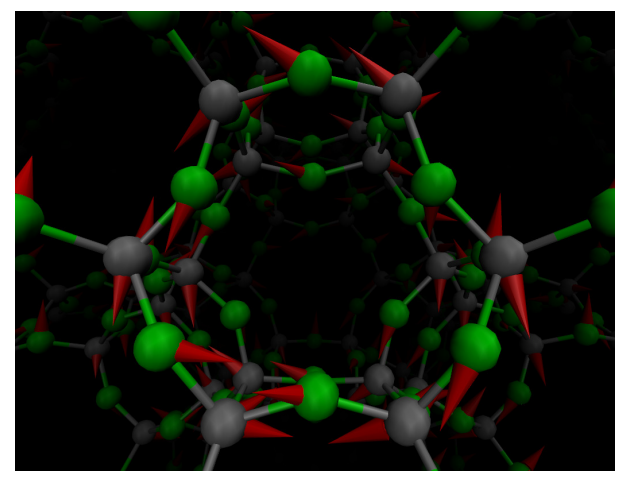

(a) The FAU IR mode at $528 \mathrm{~cm}^{-1}$

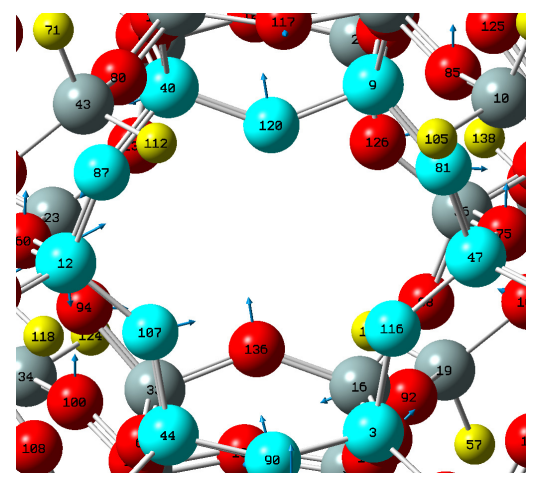

(b) The cluster IR mode at $446 \mathrm{~cm}^{-1}$

Figure 3.17. (a) The FAU IR mode at around $528 \mathrm{~cm}^{-1}$ predicted by the MZHB potential. (b) The cluster IR mode at around $446 \mathrm{~cm}^{-1}$ predicted by the cluster calculation. The molecular motions show that these two IR modes are both related to the $6 \mathrm{R}$ deformation motion, indicating that these two IR modes might match with the same experimentally observed IR peak.

Figure 3.17a presents the underlying molecular motion of the MZHB-predicted IR peak at around $528 \mathrm{~cm}^{-1}$, where the $6 \mathrm{R}$ atoms move along the circumference of the $6 \mathrm{R}$ due to the bending motion of one $\mathrm{Si}-\mathrm{O}-\mathrm{Si}$ angle. This molecular motion is also observed from the cluster calculation, which gives rise to the major peak at around $446 \mathrm{~cm}^{-1}$ in the cluster IR spectrum, shown in Figure 3.17b. Therefore, we think that this IR peak at around $528 \mathrm{~cm}^{-1}$ predicted by the MZHB potential should be assigned to the major measured peak at around $462 \mathrm{~cm}^{-1}$.

The above discussion about the peak assignments of the angle-bending peaks indicates the consistency between the MZHB-predicted FAU IR spectrum and clustercalculated IR spectrum in the sense that two $4 \mathrm{R}$ breathing modes are predicted on both sides of the major peak in the angle-bending region. Indeed, a vibrational frequency analysis performed on a 4R of the D6R unit based on MD trajectory simulated by the MZHB potential also shows that the $4 \mathrm{R}$ vibration has two breathing frequency components at around $450 \mathrm{~cm}^{-1}$ and $560 \mathrm{~cm}^{-1}$, respectively. Four-ring vibrational analysis was carried out by Fourier transform of the autocorrelation function of the 


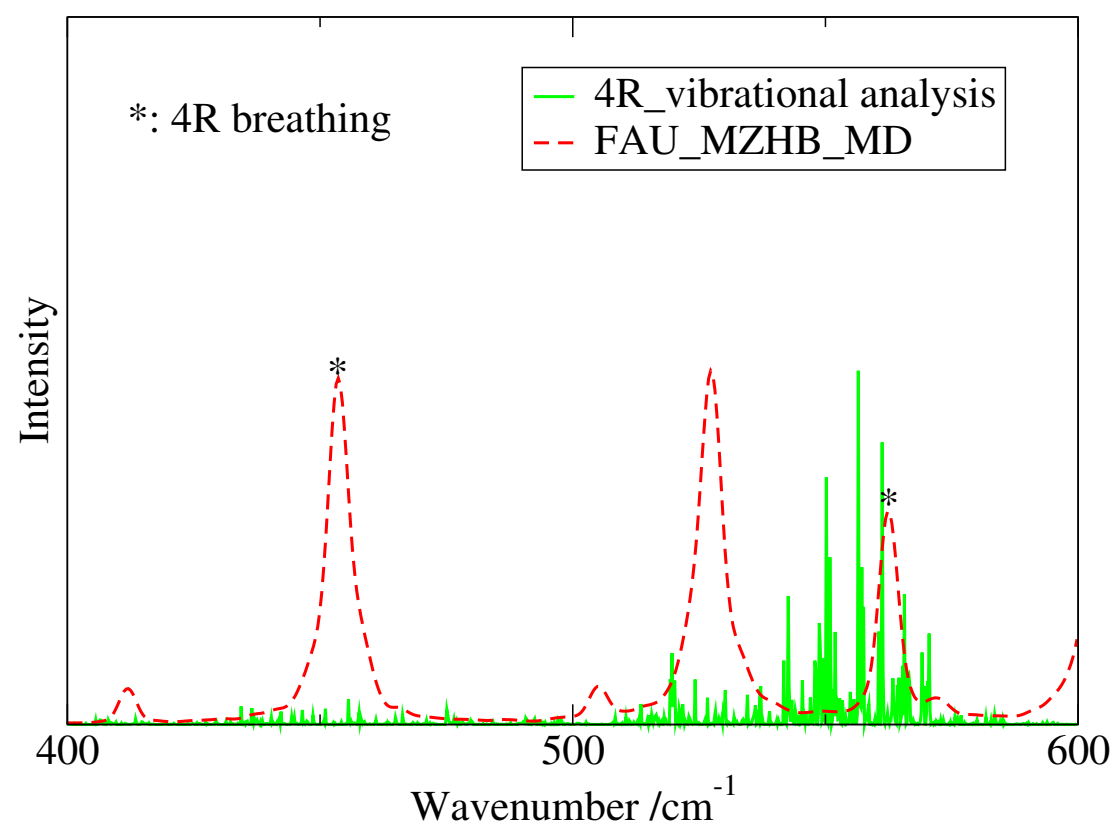

Figure 3.18. The 4-membered ring vibrational frequency analysis: two broad frequency ranges at around $450 \mathrm{~cm}^{-1}$ and $560 \mathrm{~cm}^{-1}$ are observed, these two frequency ranges are closed to the two $4 \mathrm{R}$ breathing IR modes predicted by the MZHB potential.

quantity defined in Equation (3.13); the resulting spectrum decomposes the underlying frequency components of the $4 \mathrm{R}$ vibration, presented in Figure 3.18. Note that the frequency spectrum of the $4 \mathrm{R}$ vibration is quite noisy, indicating that $4 \mathrm{R}$ vibration is actually a linear combination of vibrations of different frequencies. Nevertheless, a major frequency component is observed at around $560 \mathrm{~cm}^{-1}$, which is close to the MZHB-predicted IR peak at around $564 \mathrm{~cm}^{-1}$. A broad band with very weak intensity at around $450 \mathrm{~cm}^{-1}$ is also observed. These findings are consistent with normal mode visualizations of the angle-bending modes predicted by the MZHB potential, in the sense that two IR modes associated with the $4 \mathrm{R}$ breathing motion are predicted at around $453 \mathrm{~cm}^{-1}$ and $564 \mathrm{~cm}^{-1}$, respectively.

From the above discussion about the peak assignments of the angle-bending modes predicted by the MZHB potential, it is clear that the angle-bending modes, including two $4 \mathrm{R}$ breathing modes and a $6 \mathrm{R}$ deformation mode are shifted to higher wavenum- 


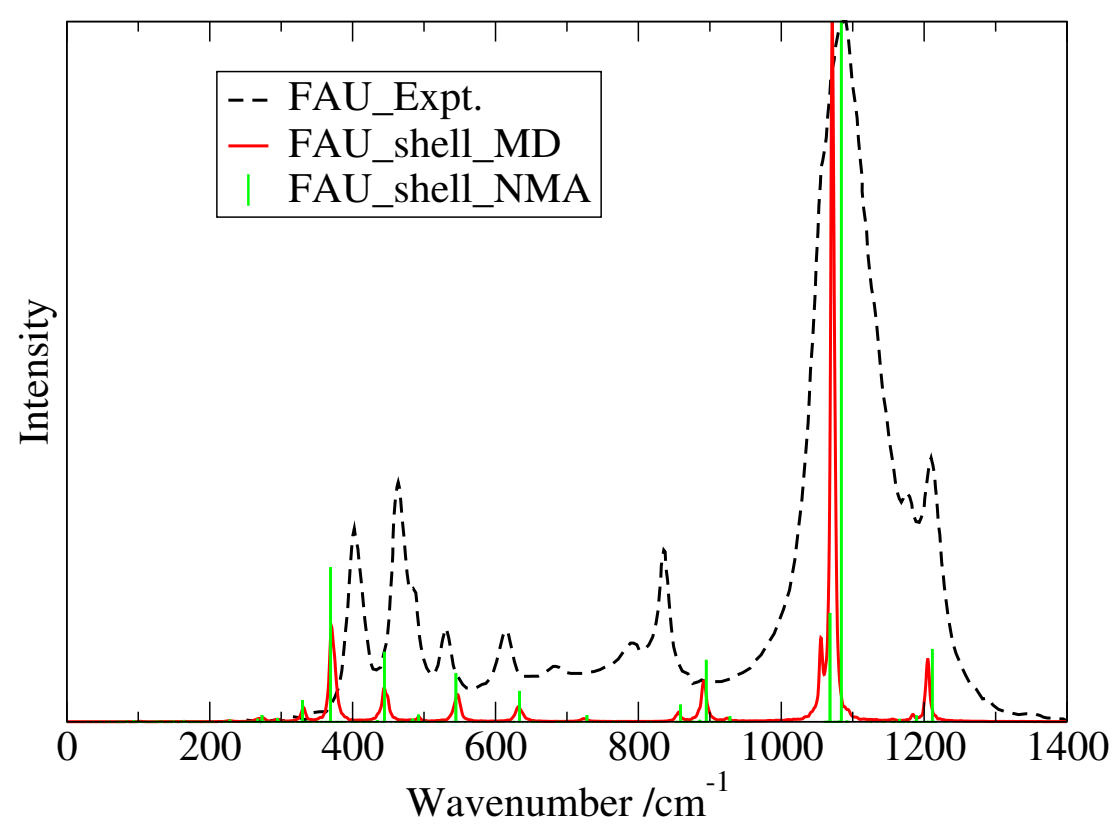

Figure 3.19. FAU IR spectra predicted by the core-shell model at $300 \mathrm{~K}$. Although the measured spectrum [104] is roughly reproduced, the underlying molecular motions associated with computed IR peaks in the angle-bending region do not agree with those from the cluster calculation. One possible reason is that the core-shell model lacks three-body interactions defined for the $\mathrm{Si}-\mathrm{O}-\mathrm{Si}$ angles, which leads to a broad $\mathrm{Si}-\mathrm{O}-\mathrm{Si}$ angle distribution and normal modes that give rise to large $\mathrm{Si}-\mathrm{O}-\mathrm{Si}$ angle deviations.

bers. These peak shifts in the angle-bending region are actually observed in the calculations for all five silica polymorphs with the MZHB potential. We believe that these shifts are due to the large $\mathrm{O}-\mathrm{Si}-\mathrm{O}$ angle-bending force constant $k_{\mathrm{O}-\mathrm{Si}-\mathrm{O}}$.

Figure 3.19 shows the FAU IR spectrum predicted by the core-shell model, compared to a measured IR spectrum [104]. Although all the experimentally observed IR peaks in the angle-bending region are reproduced in the computed IR spectrum, it is still hard to tell whether the core-shell model predicts the FAU IR spectrum correctly. For instance, based on Figure 3.12, one would expect that the underlying molecular motion of the computed IR peak predicted by the core-shell model at around $370 \mathrm{~cm}^{-1}$ would be the $4 \mathrm{R}$ breathing motion. However, when visualized, the underlying molecular motion of this mode is different from the $4 \mathrm{R}$ breathing motion. In the $4 \mathrm{R}$ breathing motion observed from the cluster calculation, three out of the 
six oxygen atoms in the same $6 \mathrm{R}$ move up and down around the $6 \mathrm{R}$ plane, as shown in Figure 3.15, while Figure 3.20 shows that in this computed mode, all six oxygen atoms in the same $6 \mathrm{R}$ move in the same direction. The underlying molecular motion of another computed IR peak at around $547 \mathrm{~cm}^{-1}$ is also inconsistent with the cluster calculation. We think that one possible reason that might explain this phenomenon is that the core-shell model lacks three-body interactions defined for the $\mathrm{Si}-\mathrm{O}-\mathrm{Si}$ angles. As is pointed out in Figure 3.10b, the lack of three-body interactions for the $\mathrm{Si}-\mathrm{O}-\mathrm{Si}$ angles results in a broad distribution of the $\mathrm{Si}-\mathrm{O}-\mathrm{Si}$ angles; we think that this may also lead to normal modes that give rise to large $\mathrm{Si}-\mathrm{O}-\mathrm{Si}$ angle deviations, hence changes the underlying molecular motions of the IR peaks in the angle-bending

region. Note that a measured IR peak at around $1210 \mathrm{~cm}^{-1}$ is reproduced by the coreshell model, which is missing in the IR spectrum predicted by the MZHB potential. This peak, according to Table 3.5, should be assigned to be a internal asymmetric stretching mode $v_{\text {as }} \mathrm{O}-\mathrm{Si}-\mathrm{O}$. Also note that computed IR peak shifts to lower wavernumbers for the peaks at around $370 \mathrm{~cm}^{-1}$ and $445 \mathrm{~cm}^{-1}$ are observed, probably due to the lack of three-body interactions defined for the $\mathrm{Si}-\mathrm{O}-\mathrm{Si}$ angles in the core-shell model, since these two modes are mainly associated with angle-bending motions. Since it is hard to match the IR peaks predicted by the core-shell model with cluster-calculated IR peaks via normal mode visualizations, peak assignments are performed empirically. Peak assignments for the predictions from both models are listed in Table 3.6.

\subsubsection{SOD (Siliceous Sodalite)}

Figure 3.21 compares the computed SOD IR spectra predicted by both the MZHB potential and the core-shell model with a measured IR spectrum [109]. All the measured peaks are roughly reproduced by both models with small peak shifts, with two major differences between the two model-predicted IR spectra. The first difference 


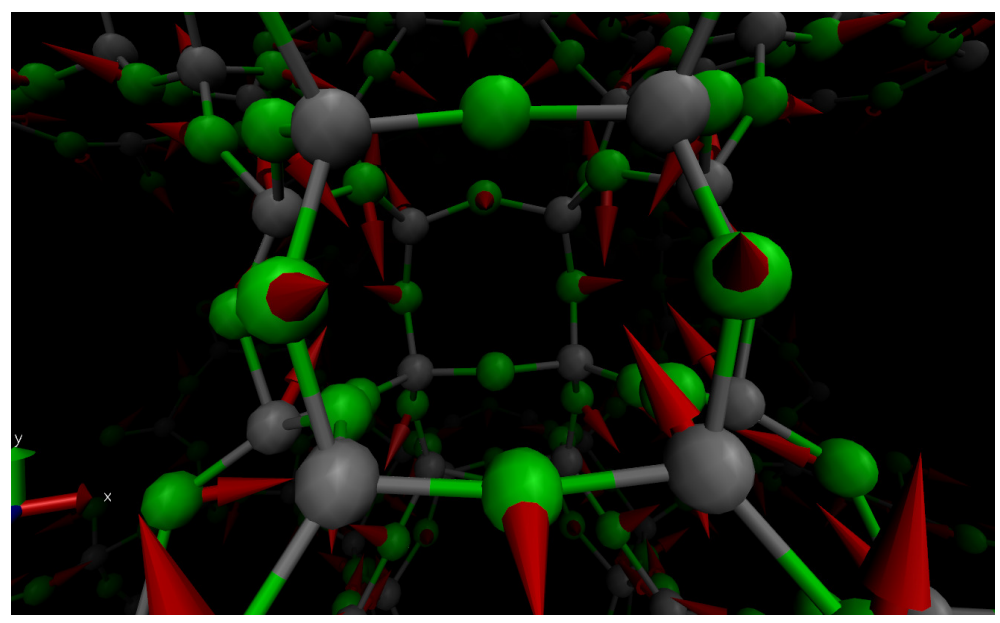

Figure 3.20. Visualization of the FAU IR peak at around $370 \mathrm{~cm}^{-1}$ predicted by the core-shell model. Although the molecular motion under this mode is similar to the $4 \mathrm{R}$ breathing motion observed from the cluster calculation, all the oxygen atoms in the same $6 \mathrm{R}$ move in the same direction with significant displacements, indicating that this mode may not be a $4 \mathrm{R}$ breathing mode.

Table 3.6. A comparison of the calculated and the measured FAU IR wavenumbers. Values are in $\mathrm{cm}^{-1}$; MD-calculated IR wavenumbers are calculated at $300 \mathrm{~K}$. Assignments (this work) were made by visualization of the normal modes.

\begin{tabular}{cccccc}
\hline \multicolumn{2}{c}{ MZHB } & \multicolumn{2}{c}{ Core-shell } & Expt. [104] & Assignment \\
\hline MD & NMA & MD & NMA & & \\
\hline 453.5 & 453.7 & 371.2 & 370.2 & 400 & Pore opening (4R breathing) \\
\hline 527.0 & 528.6 & 445.4 & 445.6 & 462 & Angle bending (6R deformation) \\
\hline 561.3 & 564.7 & & & 482 & Angle bending (4R breathing) \\
\hline & & 547.3 & 545.6 & 529 & Angle bending \\
\hline 605.8 & 606.1 & 632.2 & 634.5 & 612 & D6R \\
\hline & & 857.3 & 860.1 & 794 & External symmetric stretching \\
\hline 837.2 & 839.0 & 892.2 & 895.2 & 834 & \\
\hline 1091.0 & 1092.5 & 1072.4 & 1804.5 & 1090 & Internal asymmetric stretching \\
\hline
\end{tabular}


occurs in the angle-bending region, where a measured IR peak with medium intensity is observed at around $460 \mathrm{~cm}^{-1}$. This peak was predicted by the MZHB potential at a higher wavenumber at around $530 \mathrm{~cm}^{-1}$, which is consistent with the finding in the MZHB-predicted IR spectra for FAU in Section 3.4.2.1, that is, IR peaks in the angle-bending region are shifted to higher wavernumbers. On the other hand, the core-shell model predicted this peak at a lower wavenumber, around $438 \mathrm{~cm}^{-1}$, which is also consistent with Figure 3.19 on page 86; that is, two angle-bending modes are predicted at lower wavenumbers by the core-shell model. Another major difference occurs in the external linkage symmetric stretching region from $750-820 \mathrm{~cm}^{-1}$, where a measured IR peak with weak intensity is observed at around $788 \mathrm{~cm}^{-1}$. The core-shell model predicted a peak at a higher wavenumber around $850 \mathrm{~cm}^{-1}$, which is consistent with our interpretation in Figure 3.10a (page 75), that the core-shell model predicted a more "stiff" $\mathrm{Si}-\mathrm{O}$ bond than the MZHB potential. However, the MZHB potential gives two peaks with weak intensities in this region at around 740 and $790 \mathrm{~cm}^{-1}$, respectively. The visualization of the peak at around $740 \mathrm{~cm}^{-1}$ predicted by the MZHB potential, presented in Figure 3.22a, shows large displacements of silicon atoms, while oxygen atoms are almost stationary. This indicates that the underlying molecular motion of this mode is mainly associated with relative movements of $\mathrm{Si}$ atoms among tetrahedra, which result in symmetric $\mathrm{Si}-\mathrm{O}-\mathrm{Si}$ bond stretching motions. Bond length and angle percent changes under this mode are also plotted in Figure 3.22b. Note that the average deviations of bond angles are also significant, which means that although the $\mathrm{Si}-\mathrm{O}$ bond-stretching motion is the primary motion of this mode, strong coupling between bond length and angle deformations exist, therefore, adjustments in the angle-bending force constants $k_{\mathrm{Si}-\mathrm{O}-\mathrm{Si}}$ and $k_{\mathrm{O}-\mathrm{Si}-\mathrm{O}}$ will also shift the position of this peak significantly. Nevertheless, the predictions of the internal asymmetric stretching mode observed at around $1126 \mathrm{~cm}^{-1}$ on the 


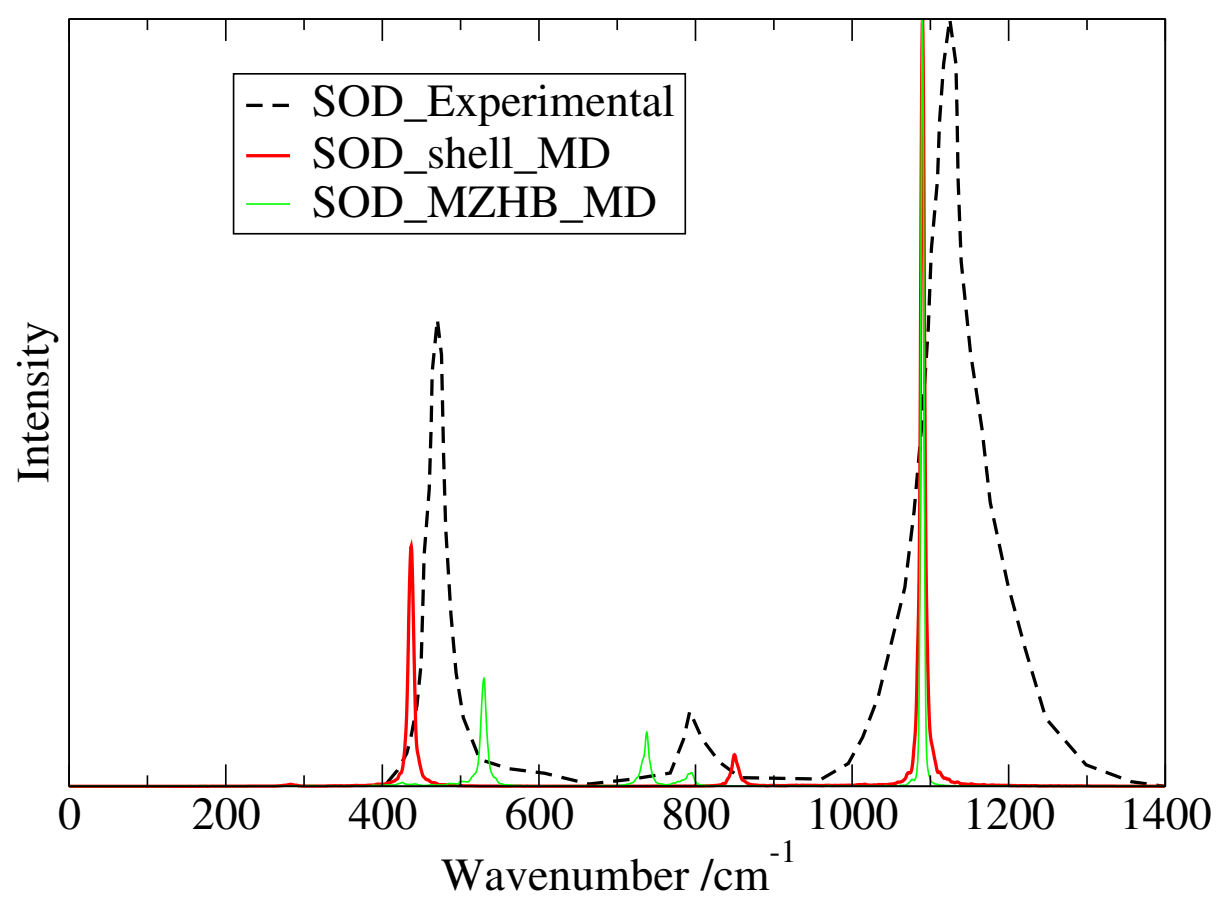

Figure 3.21. SOD IR spectra predicted by both the MZHB potential and the coreshell model at $300 \mathrm{~K}$. The MZHB potential predicts the angle-bending mode to a high wavenumber, probably because of a too large $\mathrm{O}-\mathrm{Si}-\mathrm{O}$ force constant, while the coreshell model predicts angle-bending modes to lower wavenumbers due to the lack of threebody interactions defined for the $\mathrm{Si}-\mathrm{O}-\mathrm{Si}$ angles. The measured IR spectrum is obtained from Bornhauser and Bougeard [109].

measured IR spectrum are quite close to each other: both models give wavenumbers of this peak at around $1090 \mathrm{~cm}^{-1}$. A full peak assignment is given in Table 3.7 .

\subsubsection{Quartz}

Figure 3.23 compares the model-predicted quartz IR spectra by both the MZHB potential and the core-shell model with a measured IR spectrum [110]. Obviously, all the IR peaks that are observable in the measured IR spectrum are roughly reproduced by both the two models, with peak shifts to different extents. Specifically, all the MZHB-predicted IR peaks are shifted to higher wavenumbers, and the magnitudes of shifts for peaks in the angle-bending region are much larger than those for peaks in the bond-stretching region. The core-shell model, on the other hand, predicts 


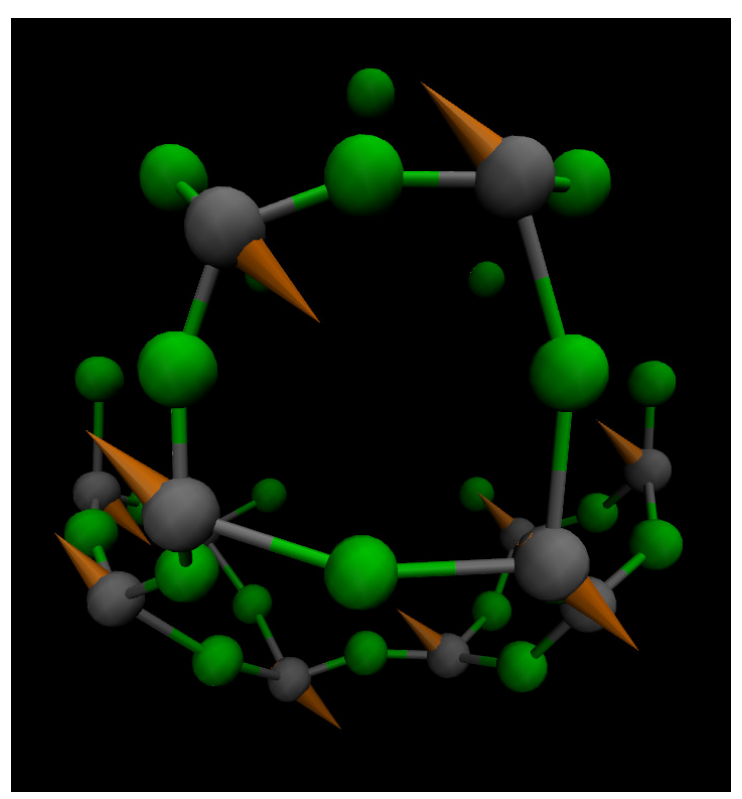

(a) Visualization of the SOD peak at around $740 \mathrm{~cm}^{-1}$.

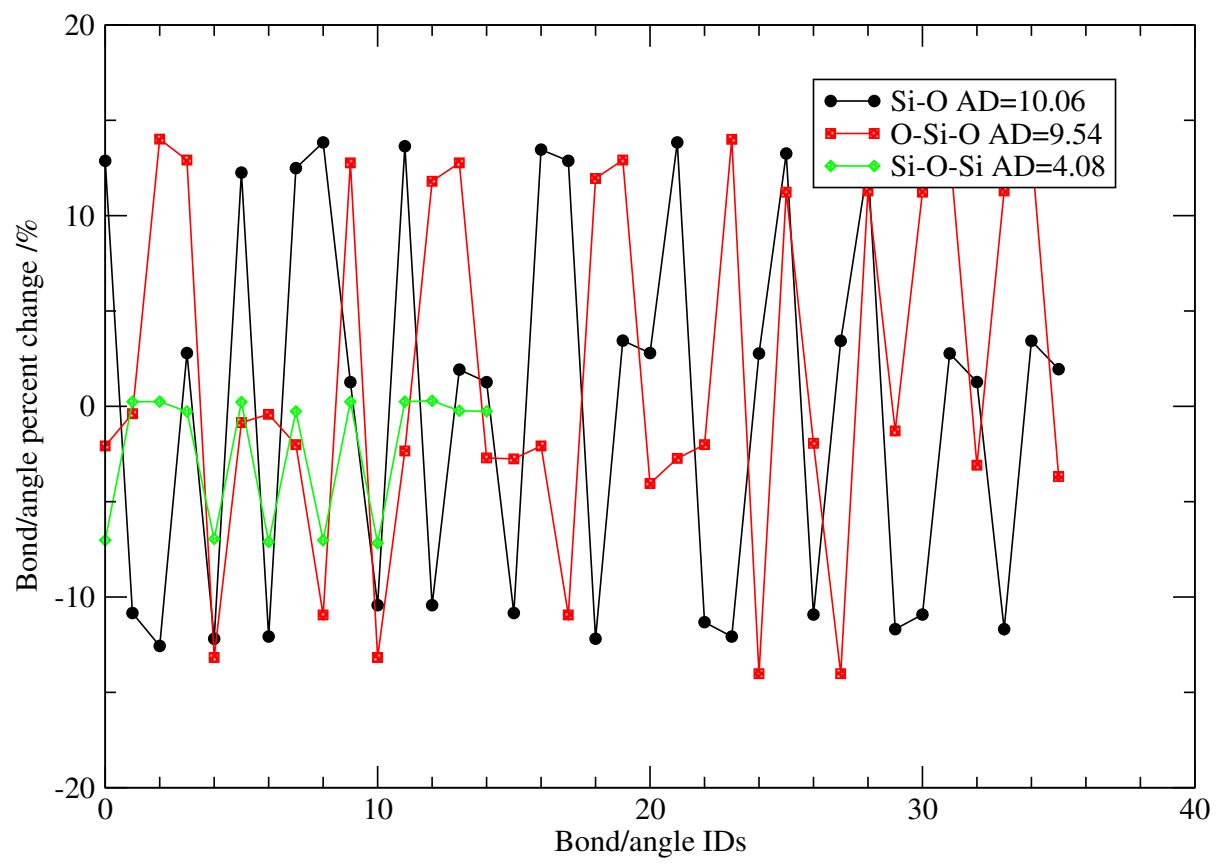

(b) Bond length and angle percent changes of the SOD peak at around $740 \mathrm{~cm}^{-1}$

Figure 3.22. (a) Visualization of the SOD peak at around $740 \mathrm{~cm}^{-1}$ : gray for $O$, green for $\mathrm{Si}$, arrows indicate the direction of most significant atomic displacements. It is clear that $\mathrm{Si}-\mathrm{O}$ bond symmetric stretching is the primary motion of this mode. (b) Bond length and angle percent changes of the SOD peak at around $740 \mathrm{~cm}^{-1}$. Average deviation (AD) values indicate that although $\mathrm{Si}-\mathrm{O}$ bond-stretching is the primary motion of this mode, strong coupling effects between bond length and angle deformations exist, indicating significant dependence of this peak position on angle-bending force constants. 
Table 3.7. A comparison of the MD-calculated and the measured SOD IR wavenumbers. Values are in $\mathrm{cm}^{-1}$; MD-calculated IR wavenumbers are calculated at $300 \mathrm{~K}$.

\begin{tabular}{cccc}
\hline MZHB & Core-shell & Expt. [109] & Assignment \\
\hline 530.5 & 438.5 & 460 & Angle bending \\
\hline 740.3 & 852.3 & 788 & External symmetric stretching \\
795.4 & & & Internal asymmetric stretching \\
\hline 1090.5 & 1090.3 & 1126 & \\
\hline
\end{tabular}

angle-bending modes shifted to lower wavenumbers and external linkage symmetric stretching modes shifted to higher wavenumbers. These two observations from the predictions of the MZHB potential and the core-shell model are consistent with the findings in both the case of FAU and SOD, shown in Figures 3.13, 3.19 and 3.21. Indeed, these observations are common to all five types of silica polymorphs studied in this work.

As has been observed in the SOD IR spectrum predicted by the MZHB potential in Figure 3.21, two external linkage symmetric stretching modes due to the motions of centered Si atoms are also observed in the MZHB-predicted IR spectrum for quartz, from $700-800 \mathrm{~cm}^{-1}$. When visualized, both the molecular motions associated with these two modes are $\mathrm{Si}-\mathrm{O}-\mathrm{Si}$ symmetric stretching; the difference between the two modes lies in the directions of the atomic displacements of Si atoms. We speculate that these two peaks may split from one peak at this wavenumber region due to the symmetry of the structure. Note that a weak IR peak at around $1161 \mathrm{~cm}^{-1}$ is predicted by the core--shell model, which matches with the shoulder at around $1147 \mathrm{~cm}^{-1}$ in the measured IR spectrum. This shoulder is also observed in the measured IR spectra of other silica polymorphs, such as FAU, in Figure 3.13. One possible explanation of the appearance of this shoulder is that this shoulder is originated from aluminum composition [104], as discussed in Section 3.4.2.1. On the other hand, it is also possi- 


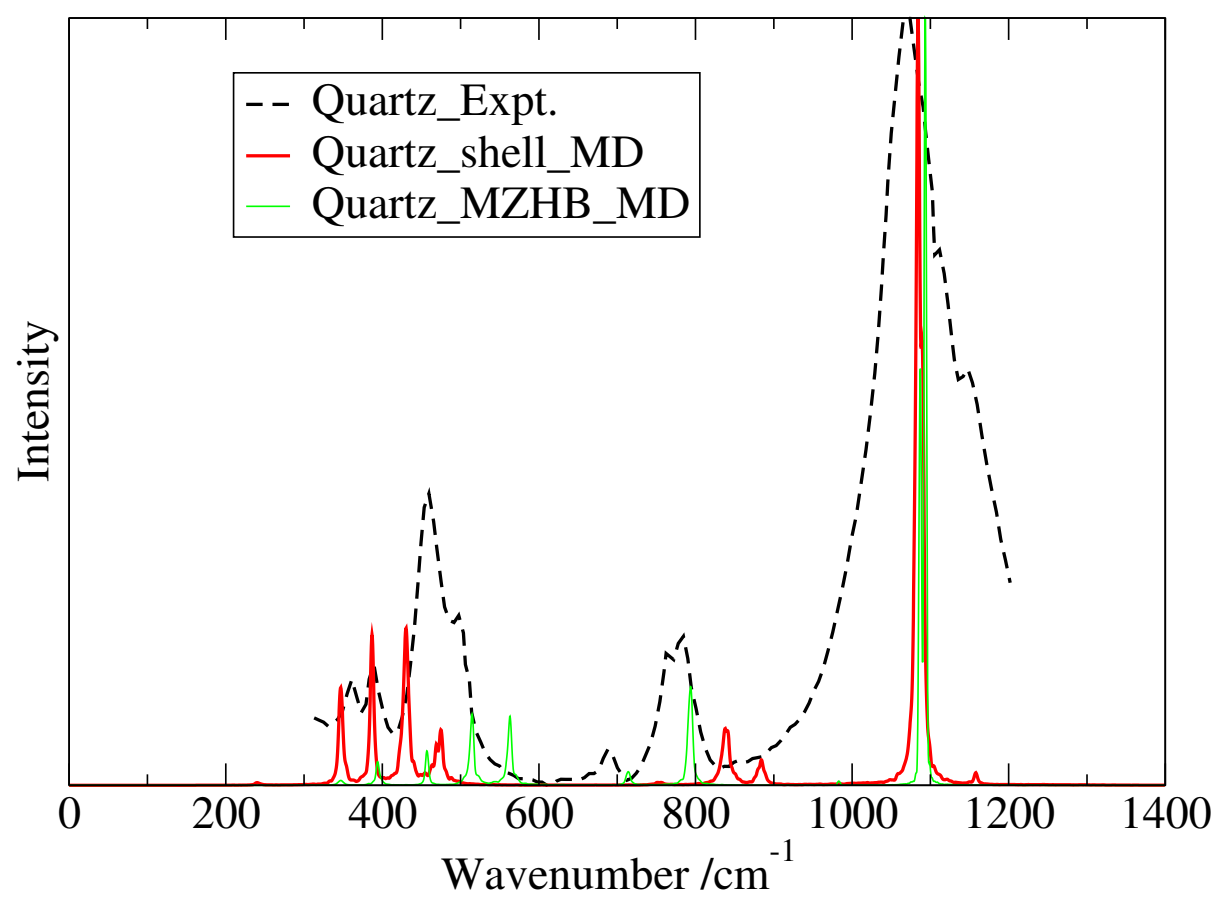

Figure 3.23. Quartz IR spectra predicted by both the MZHB potential and the core-shell model at $300 \mathrm{~K}$. The MZHB-predicted peaks shift to higher wavenumbers with different magnitudes, while the core-shell model predicts angle-bending modes at lower wavenumbers and external linkage symmetric modes at higher wavernumbers. A weak shoulder is predicted at $1161 \mathrm{~cm}^{-1}$ by the core-shell model, which probably can be assigned to an asymmetric stretching mode. The measured IR spectrum is from Liang et al. [110].

ble that this shoulder is an internal asymmetric bond stretching mode, splitting from the main peak $[22,102]$. A full peak assignment is presented in Table 3.8. IR peaks predicted by the core-shell model match better with the measured IR peaks.

\subsubsection{Cristobalite}

Figure 3.24 compares the computed cristobalite IR spectra predicted by both the MZHB potential and the core-shell model with a measured IR spectrum [110]. Obviously the MZHB potential predicts angle-bending modes shifted to higher wavenumbers in between $400-600 \mathrm{~cm}^{-1}$, and the peak predicted by the core-shell model shifts to a lower wavenumber at around $438 \mathrm{~cm}^{-1}$. Note that while the internal asymmetric stretching mode is well reproduced by both the potentials, the core-shell model fails 
Table 3.8. A comparison of the MD-calculated and the measured quartz IR wavenumbers. Values are in $\mathrm{cm}^{-1}$; MD-calculated IR wavenumbers are calculated at $300 \mathrm{~K}$.

\begin{tabular}{cccc}
\hline MZHB & Core-shell & Expt. [110] & Assignment \\
\hline 394.6 & 349.3 & 363.0 & \\
458.3 & 388.8 & 388.8 & Angle bending \\
515.7 & 431.7 & 459.1 & \\
563.3 & 476.3 & 498.6 & \\
\hline & 754.2 & 690.7 & Internal symmetric bond stretching \\
\hline 714.1 & 840.0 & 764.5 & External symmetric stretching \\
793.7 & 886.3 & 786.8 & \\
\hline \multirow{2}{*}{1095.6} & 1087.1 & 1071.6 & Internal asymmetric stretching \\
& 1162.3 & 1147.3 & \\
\hline
\end{tabular}

Table 3.9. A comparison of MD-calculated and measured cristobalite IR wavenumbers. Values are in $\mathrm{cm}^{-1}$; MD-calculated IR wavenumbers are calculated at $300 \mathrm{~K}$.

\begin{tabular}{cccc}
\hline MZHB & Core-shell & Expt. [110]. & Assignment \\
\hline 555 & 438 & 472 & Angle bending \\
586 & 443 & & Angle bending \\
\hline 615 & & 609 & External symmetric stretching \\
\hline 771 & 849 & 787 & Internal asymmetric bond stretching \\
\hline 1089 & 1110 & 1086 & \\
\hline
\end{tabular}

to predict the external linkage symmetric stretching mode at around $787 \mathrm{~cm}^{-1}$ and the peak at around $609 \mathrm{~cm}^{-1}$. A full peak assignment is given in Table 3.9.

\subsubsection{MFI (Silicalite-1)}

Figure 3.25 compares the computed IR spectra of silicalite-1 (siliceous MFI) predicted by both the MZHB potential and the core-shell model with a measured IR spectrum [111]. The consistent shift of angle-bending modes to higher wavenumbers is observed in the spectrum predicted by the MZHB potential, leaving only one broad band in the range between $400-600 \mathrm{~cm}^{-1}$. This merging of two experimentally observed peaks might be because of the fact that the positions of these two peaks have 


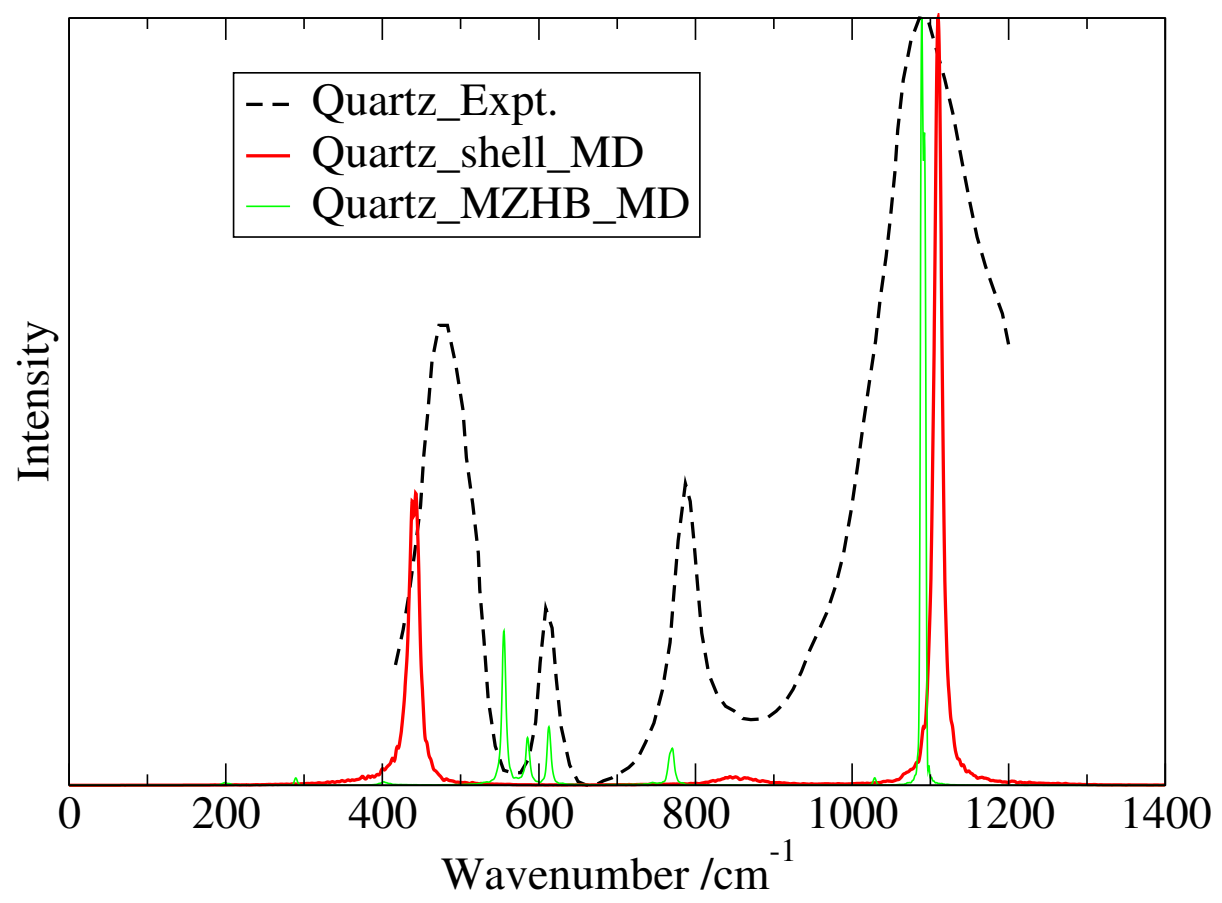

Figure 3.24. Cristobalite IR spectra predicted by both the MZHB potential and the coreshell model at $300 \mathrm{~K}$. Apparent peak shifts to higher wavenumbers and lower wavenumbers in the angle-bending region are observed, predicted by the MZHB potential and the coreshell model, respectively. The core-shell model fails in the prediction of the external linkage symmetric stretching mode at around $787 \mathrm{~cm}^{-1}$ and the peak at around $609 \mathrm{~cm}^{-1}$. The measured spectrum is obtained from Liang et al. [110].

different sensitivities to the angle-bending force constants. These two experimentally observed peak ranges, 400 to $500 \mathrm{~cm}^{-1}$ and 500 to $600 \mathrm{~cm}^{-1}$, might be assigned to an angle-bending mode and a double ring vibrational mode, respectively, according to Table 3.5. Thus, it is possible that the angle-bending mode shifts to a high wavenumber more dramatically than the double ring vibrational mode, resulting in the merging of these two peaks. However, the internal asymmetric stretching mode at around $1096 \mathrm{~cm}^{-1}$ and the external symmetric stretching mode at around $799 \mathrm{~cm}^{-1}$ are well-reproduced by the MZHB potential. On the other hand, two broad bands, slightly shifted to lower wavenumbers, are observed in the computed IR spectrum predicted by the core-shell model at around 400 and $550 \mathrm{~cm}^{-1}$, which are consistent with the measured IR spectrum [111]. While the internal asymmetric stretching 


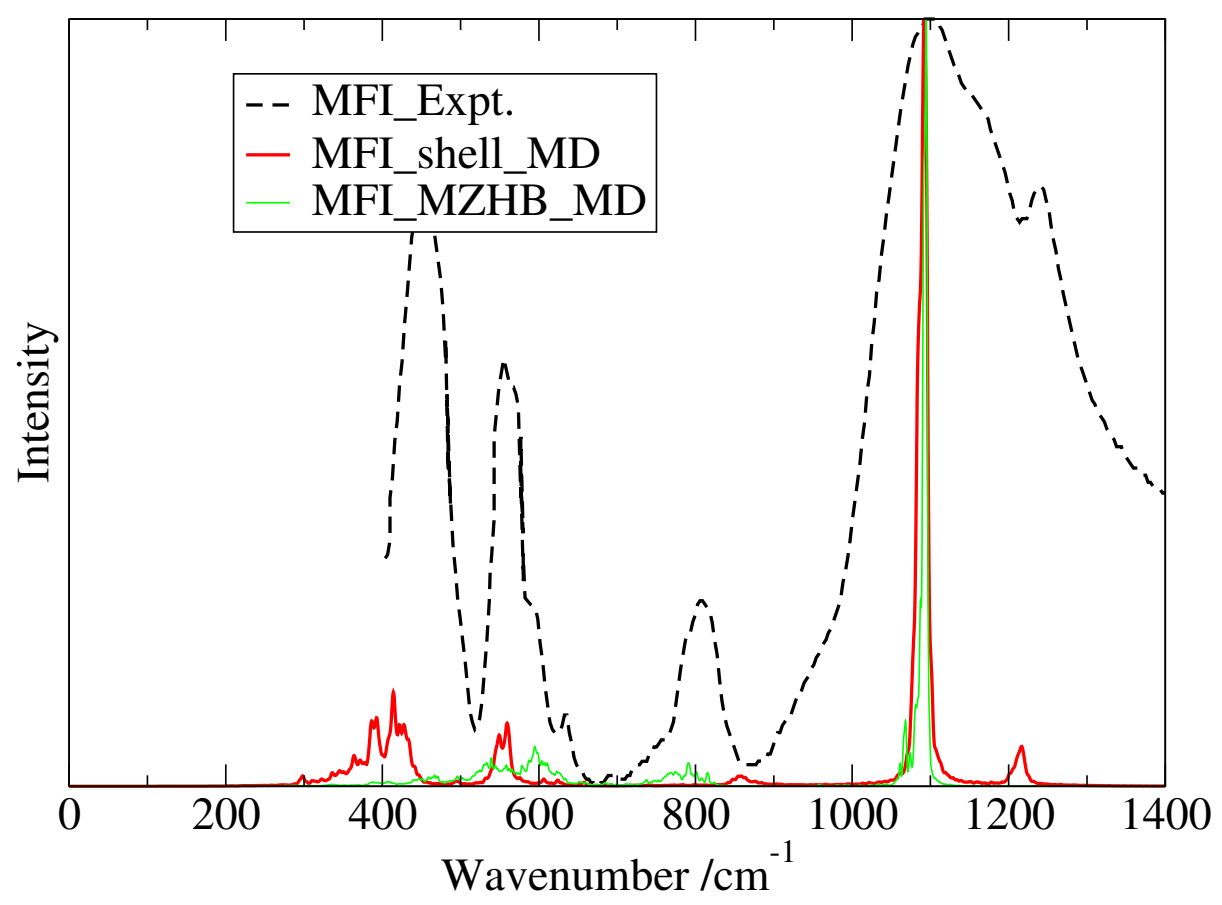

Figure 3.25. MFI IR spectra predicted by both the MZHB potential and the coreshell model at $300 \mathrm{~K}$ : while the core-shell model predicts clearly two broad IR bands at around 400 and $550 \mathrm{~cm}^{-1}$, respectively, the MZHB potential predicts only one broad band in the region ranges from 400 to $600 \mathrm{~cm}^{-1}$, probably due to the difference of the sensitivities of these two peak's positions to the angle-bending force constants; however, the external linkage symmetric stretching mode predicted by the MZHB potential matches better with the measured peak at around $800 \mathrm{~cm}^{-1}$. The measured IR spectrum is obtained from Serrano et al. [111].

modes at around $1108 \mathrm{~cm}^{-1}$ and $1226 \mathrm{~cm}^{-1}$ are well-reproduced, the external linkage symmetric stretching mode shifts to a higher wavenumber.

\subsection{The Lennard-Jones Parameters of the MZHB Potential}

In the original work of the MZHB potential by Sahoo and Nair [21], the LennardJones parameters of the MZHB potential were obtained from Zimmerman et al. [27], in which parameterization of the atomic partial charges and Lennard-Jones parameters for siliceous zeolite framework silicon and oxygen atoms were carried out via fitting to the heats of adsorption of small gas molecules and the cracking activation energies of hydrocarbons. The target heats of adsorption and activation energies 
Table 3.10. The Lennard-Jones parameters used in the MZHB potential [21].

\begin{tabular}{ccc}
\hline Atomic species & $r_{m}(\AA)$ & $\epsilon(\mathrm{eV})$ \\
\hline $\mathrm{Si}$ & 2.200 & 0.00864 \\
$\mathrm{O}$ & 1.770 & 0.00324 \\
\hline
\end{tabular}

Table 3.11. The Lennard-Jones parameters published in the work of Lopes et al. [32]. Note the factor of 2 that is missing from Table 3.10.

\begin{tabular}{ccc}
\hline Atomic species & $r_{m} / 2(\AA)$ & $\epsilon(\mathrm{kcal} / \mathrm{mol})$ \\
\hline $\mathrm{Si}$ & 2.200 & 0.6000 \\
$\mathrm{O}$ & 1.770 & 0.3021 \\
\hline
\end{tabular}

were calculated with full quantum mechanical calculations with density functional theory, atomic partial charges and Lennard-Jones parameters are then adjusted in quantum mechanics/molecular mechanics (QM/MM) calculations to reproduce these target data. Note that in their fitting procedure, only atomic partial charges for oxygen and silicon atoms and the Lennard-Jones energy parameters, $\epsilon_{i}$, are adjusted, while the minimum-energy internuclear distances, $r_{m, i}$, are fixed. The initial values of these parameters were obtained from Lopes et al. [32]. Formulas used for molecular mechanics calculations are the same as those implemented in the MZHB potential, given by Equations (3.3) and (3.4). The combination rules for Lennard-Jones parameters are the arithmetic mean for $r_{m}$ and the geometric mean for $\epsilon$. The resulting optimized Lennard-Jones parameters, which were then adopted by the MZHB potential, are listed in Table 3.10. However, the values of $r_{m}$ for both oxygen and silicon in Table 3.10 were not consistent with the values published in the work of Lopes et al. [32], as listed in Table 3.11.

It is clear that the difference between the two parameter sets is that: in Table 3.10, $r_{m}$ for $\mathrm{Si}$ and $\mathrm{O}$ are $2.2000 \AA$ and $1.770 \AA$, respectively; while in Table $3.11, r_{m}$ for $\mathrm{Si}$ and $\mathrm{O}$ are $4.400 \AA$ and $3.540 \AA$, respectively. Therefore, an apparent transposition 
error is observed here. This transposition error actually may answer the question as to why the MZHB potential does not exclude the long-range interactions between valence atom pairs, as typical CHARMM-style potentials [30] do, but still shows reasonable performance in predicting silica polymorphs' geometries.

Generally speaking, as discussed in Chapter 2, for a CHARMM-style potential [30], the long-range interactions between atom pairs separated by one bond (1-2 atom pairs) and two bonds (1-3 atom pairs) are excluded, since their interactions have been covered by bond interactions and angle interactions; and the long-range interactions between atom pairs separated by three bonds (1-4 atoms pair) are scaled since their interactions have been partially covered by torsional interactions. If these interactions are not excluded, the simulated structure might expand unreasonably due to strong repulsions. This is illustrated in Figure 3.26a, in which the Lennard-Jones energy profiles for $\mathrm{O}-\mathrm{O}, \mathrm{Si}-\mathrm{O}$ and $\mathrm{Si}-\mathrm{Si}$ atom pairs are plotted (the energies are scaled so the well is the same depth in each case, for the purpose of discussion). The inter-atomic distances are based on the corresponding radial distribution functions, discussed in Section 3.4.1.1. It is clear that the interactions between the closest $\mathrm{Si}-\mathrm{O}$ and $\mathrm{Si}-\mathrm{Si}$ atom pairs are both repulsive, which tend to expand the simulated structure, if not excluded. It also explains why typical CHARMM-style potential will exclude the long-range interactions between these atom pairs. However, the LennardJones energy profiles are different for the MZHB potential due to the transposition error, presented in Figure 3.26b. Here we observed that although the interactions between the closest $\mathrm{Si}-\mathrm{O}$ atom pairs are repulsive, the interactions between the closest $\mathrm{Si}-\mathrm{Si}$ atom pairs are attractive, which means that it is possible to achieve a local force balance in such a system. We think such a local force balance may answer the question why the MZHB potential does not exclude the long-range interactions between valence atom pairs, but still has a good performance in predicting silica polymorphs' geometries. 

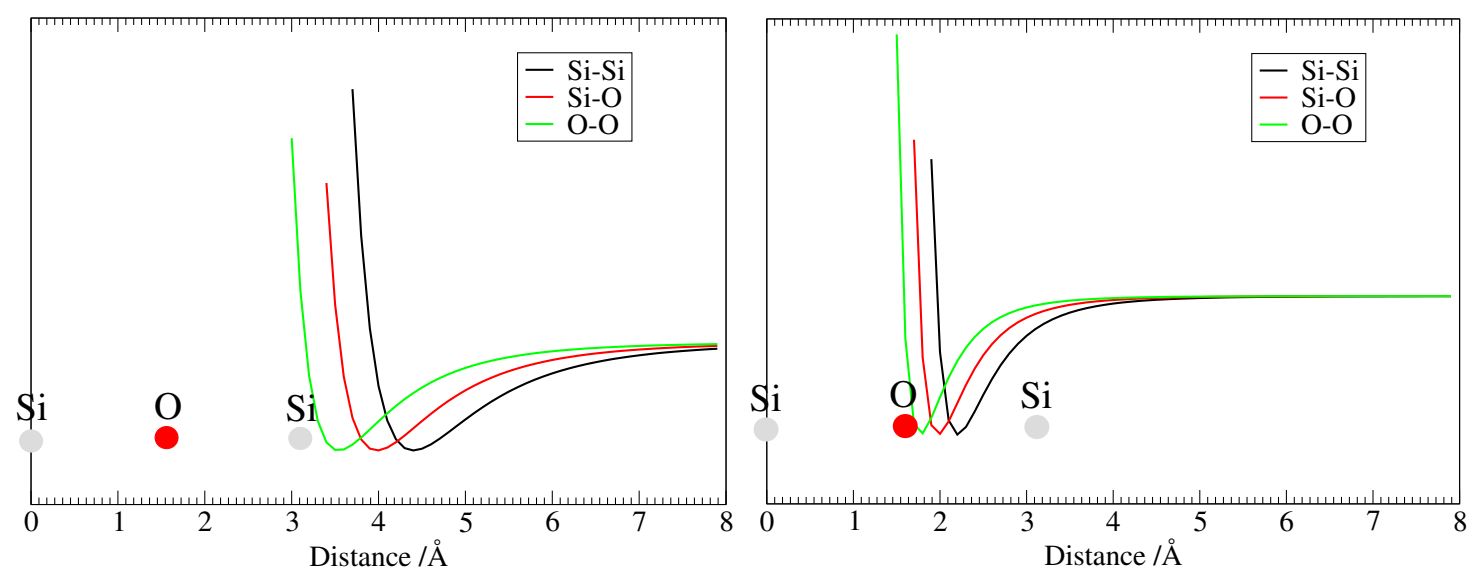

(a) The Lennard-Jones energy profiles using (b) The Lennard-Jones energy profiles usparameters from Lopes et al. [32] ing parameters from Zimmerman et al. [27]

Figure 3.26. A comparison of the Lennard-Jones potential using parameters from CHARMM (Lopes et al. [32]) and from MZHB (Sahoo and Nair [21]). The MZHB parameters were inherited from Zimmerman et al. [27].

\subsection{Conclusion}

In this chapter, two published potentials of different functional forms are tested in terms of their abilities to predict silica polymorphs' equilibrium geometries, bulk moduli, and IR spectra. The comparison of the model-predicted silica polymorphs' equilibrium geometries with experimental observables shows that the MZHB potential is generally more capable of predicting equilibrium geometries, while the core-shell model predicts a larger average $\mathrm{Si}-\mathrm{O}-\mathrm{Si}$ angle and a broader angle distribution, possibly because of its lack of three-body interactions to constrain the deformation of the $\mathrm{Si}-\mathrm{O}-\mathrm{Si}$ angles during molecular dynamics simulations. We think this also results in normal modes that give rise to large $\mathrm{Si}-\mathrm{O}-\mathrm{Si}$ angle deviations, hence making it harder to assign IR peaks based on normal mode visualizations. The narrower equilibrium bond distribution predicted by the core-shell model indicates that the core-shell model predicts a "stiffer" bond compared with the MZHB potential. These observed phenomena in silica polymorphs' equilibrium geometries are consistent with 
our findings in the computed IR spectra predicted by the core-shell model, in the sense that consistent shifts of the angle-bending modes toward lower wavenumbers and the external linkage symmetric stretching modes towards higher wavenumbers are observed in all five types of silica polymorphs studied in this work. On the other hand, although the MZHB potential shows its transferability to reproduce silica polymorphs' geometric observables, we also observe that this potential consistently shifts anglebending modes to higher wavenumbers for all five types of silica polymorphs studied here. This has been confirmed by normal mode analysis, the $4 \mathrm{R}$ vibrational frequency analysis, and quantum mechanical cluster calculations. This consistent peak shift indicates that the $\mathrm{O}-\mathrm{Si}-\mathrm{O}$ angle-bending force constant of the MZHB potential is too large. Cluster calculations combined with the normal mode analysis may offer a systematic way to understand the underlying molecular motions of angle-bending modes. We also point out that the Lennard-Jones parameters of the MZHB potential are not well-grounded due to a transposition error as we discussed in Section 3.5. 


\section{CHAPTER 4}

\section{NEW POTENTIAL}

As discussed in Chapter 3, which compares the performance of the MZHB potential [21] and the core-shell model [22] in terms of predicting IR spectra for five silica polymorphs, we observed that although the MHZB potential consistently shifts the angle-bending modes to higher wavenumbers, the underlying molecular motions of these angle-bending modes are quite consistent with the results from the cluster calculation; while it is very hard to make such matches for the angle-bending modes predicted by the core-shell model due to its lack of $\mathrm{Si}-\mathrm{O}-\mathrm{Si}$ three-body interactions. Despite the issue with the Lennard-Jones parameter $r_{m}$, discussed in Section 3.5, the MZHB potential also predicts narrower equilibrium angle distributions and average geometric observables closer to the corresponding experimental values. Therefore, in order to fix the peak shift problem, in this chapter we re-parameterize the MZHB potential and develop a new potential. Model parameters of the new potential are derived by first performing a sensitivity analysis investigating the dependence of the siliceous zeolite lattice parameter on the MZHB model parameters; the bondstretching and angle-bending force constants are then adjusted to fit to the FAU experimental lattice parameter, bulk modulus, and IR spectrum. The performance of the new potential is also tested and compared to that of the original MZHB potential to demonstrate that the new potential better reflects the siliceous zeolite framework interactions. 


\subsection{Sensitivity Analysis of the MZHB Potential}

A sensitivity analysis investigating the dependence of the siliceous zeolite bulk modulus and lattice parameter at $0 \mathrm{~K}$ on the MZHB model parameters is performed via GULP [29]. The bulk modulus is calculated with Equation (4.1),

$$
K=\frac{1}{9}\left(C_{11}+C_{22}+C_{33}+2\left(C_{12}+C_{13}+C_{23}\right)\right),
$$

where $C_{i, j}$ denotes element $(i, j)$ of the stiffness tensor. Sensitivity analysis is helpful in the sense that it offers rough ideas and guidance about the parameters that should be modified when altering a published model. Three siliceous zeolites, including FAU (siliceous zeolite Y), SOD (siliceous sodalite) and LTA (siliceous zeolite A) were studied to perform the sensitivity analysis. A change from negative $5 \%$ to positive 5\% was applied to each model parameter for FAU and SOD; a $10 \%$ change was made for LTA. The adjustable parameters are the force constants for covalent interactions, the atoms' partial charges, and the Lennard-Jones energy parameters used for computing long-range interactions. The reason we did not change the equilibrium parameters $R_{0}$ and $\theta_{0}$ is because these parameters are obtained directly from quantum mechanical calculations or experimental measurements in most cases [19, 20]. Also, since Lennard-Jones interactions are supposed to represent van der Waals interactions and electron-electron repulsion, the minimum energy inter-nuclear distance, $r_{m}$, is a product of the balance of these two forces between the two involved atoms $[112,113]$. This minimum energy inter-nuclear distance is approximately the sum of the van der Waals radius of the two involving atoms. For example, the measured van der Waals radius for a silicon atom in the work of Bondi [112] is $2.10 \AA$, and the $r_{m}$ for $\mathrm{Si}-\mathrm{Si}$ atom pairs used in the work of Lopes et al. [32] is $4.40 \AA$.

Figure 4.1 shows the effect of each adjusted parameter on the lattice parameter. Similar trends in the lattice parameter with respect to each of the adjusted param- 
eters among all three zeolite frameworks are observed. Obviously, changes in the $\mathrm{Si}-\mathrm{O}$ bond-stretching force constant and atomic partial charges have the most significant effects on zeolite lattice parameter, whereas the Lennard-Jones parameters have little effect on the lattice parameter. This significant dependence of the lattice parameter on partial atomic charges and the $\mathrm{Si}-\mathrm{O}$ bond-stretching force constant indicates that one should adjust these two model parameters in order to obtain lattice parameters closer to experimental values. Note that the force constants of internal coordinates, such as $k_{\mathrm{Si}-\mathrm{O}}$ for bond-stretching and $k_{\mathrm{O}-\mathrm{Si}-\mathrm{O}}$ for angle-bending, are typically responsible for local molecular geometries, since these force constants are mostly derived from the Hessian matrix obtained from quantum mechanical calculations [114, 115], vibrational data, and experimentally measured bond lengths and angles. Therefore, partial atomic charges may be good candidates to adjust to fit crystal lattice parameters. As discussed in Section 2.1.4.2, partial atomic charges are often determined via fitting to quantum-mechanically-calculated energy maps based on hydrogen-terminated clusters (which we will call the potential energy surface, or PES, Method) [72, 73], or determined via fitting to the energy and equilibrium distance of individual atom-water interacting pairs (Supramolecule Method) [31, 32, 69], neither of which takes into account the effect of periodic boundary conditions, that is, the energy contributed from the atomic interactions between the studied cluster and its periodic images. Periodic boundary conditions, however, are fundamental aspects of crystalline material simulations. Thus, for a crystalline material with periodic boundary conditions, one might consider finding partial atomic charges via fitting to crystal lattice parameters, or more practically, fine-tuning the partial atomic charges derived from cluster calculations via fitting to the crystal lattice parameter.

Figure 4.2 presents the effect of each of the adjusted parameters on the zeolite bulk modulus. One can tell that covalent interactions like $\mathrm{Si}-\mathrm{O}$ bond-stretching interactions and $\mathrm{O}-\mathrm{Si}-\mathrm{O}$ angle-bending interactions play more important roles than 


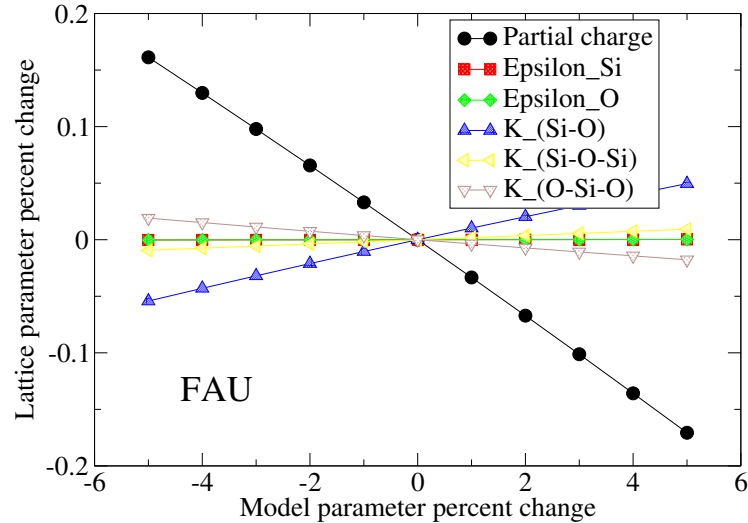

(a) Sensitivity analysis of the FAU lattice parameter at $0 \mathrm{~K}$.

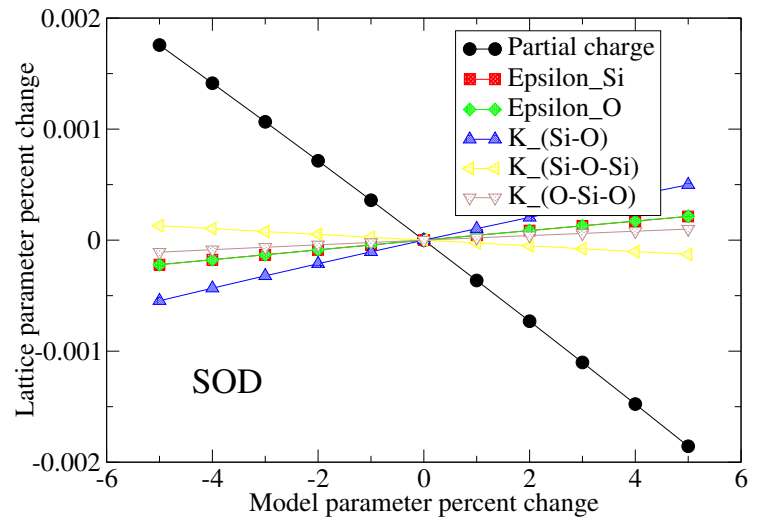

(b) Sensitivity analysis of the SOD lattice parameter at $0 \mathrm{~K}$.

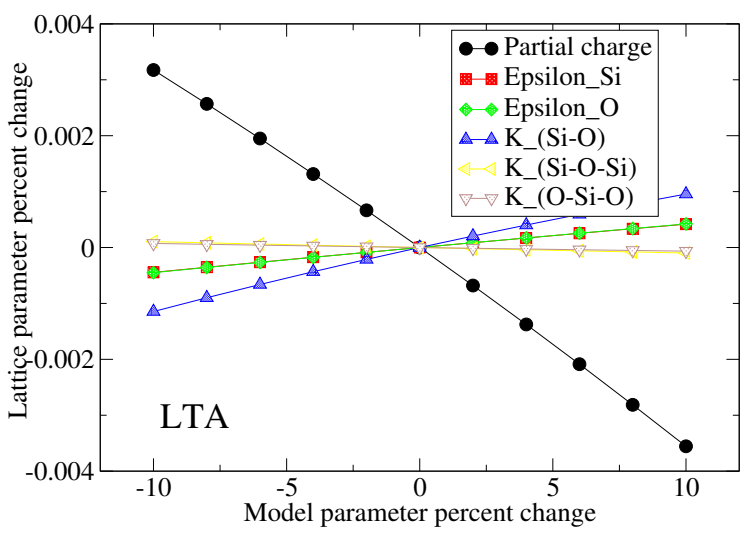

(c) Sensitivity analysis of the LTA lattice parameter at $0 \mathrm{~K}$.

Figure 4.1. Sensitivity analysis of the siliceous zeolite lattice parameter dependence on model parameters at $0 \mathrm{~K}$. The $\mathrm{Si}-\mathrm{O}$ bond force constant and partial atomic charges have the most significant effects on the siliceous zeolite lattice parameter. 
long-range interactions in the calculation of the zeolite bulk modulus. This is actually expected, since the bulk modulus can be computed from the elastic constants, and the elastic constants are actually the second derivatives of the free energy with respect to strain components, which are related to the Hessian matrix [29].

\subsection{The New Potential}

With the help of the above sensitivity analysis of the MZHB model parameters, a new potential has been developed on the basis of the MZHB potential, by fitting to the FAU experimental lattice parameter, bulk modulus, and IR spectrum via adjusting the bond-stretching and angle-bending force constants. The functional forms of the new potential are listed below. Note that in addition to the functional forms appearing in the original MZHB potential, a Urey-Bradley potential term is included, given by Equation (4.4). As another way to account for bond length and angle coupling effects, the Urey-Bradley potential term is added to distinguish subtleties in vibrational spectra; for example, symmetric and asymmetric bond-stretching vibrational frequencies [20]. Model parameters of the new potential are presented in Table 4.1. Equilibrium bond length and angle parameters $\left(r_{0}\right.$ and $\left.\theta_{0}\right)$ are inherited from the MZHB potential [21]. The Urey-Bradley potential parameters are obtained from Nicholas et al. [20]. The Lennard-Jones parameters and atomic partial charges are obtained from Lopes et al. [32] with a cutoff distance of $12 \AA$.

$$
\begin{gathered}
V_{\text {bond }}=\frac{1}{2} k_{R}\left(R-R_{0}\right)^{2} \\
V_{\text {angle }}=\frac{1}{2} k_{\theta}\left(\theta-\theta_{0}\right)^{2} \\
V_{\mathrm{UB}}=\frac{1}{2} k_{\mathrm{UB}}\left(r-r_{0}\right)^{2} \\
V_{\mathrm{EL}}=\frac{1}{4 \pi \epsilon_{0}} \frac{q_{i} q_{j}}{r_{i j}}
\end{gathered}
$$



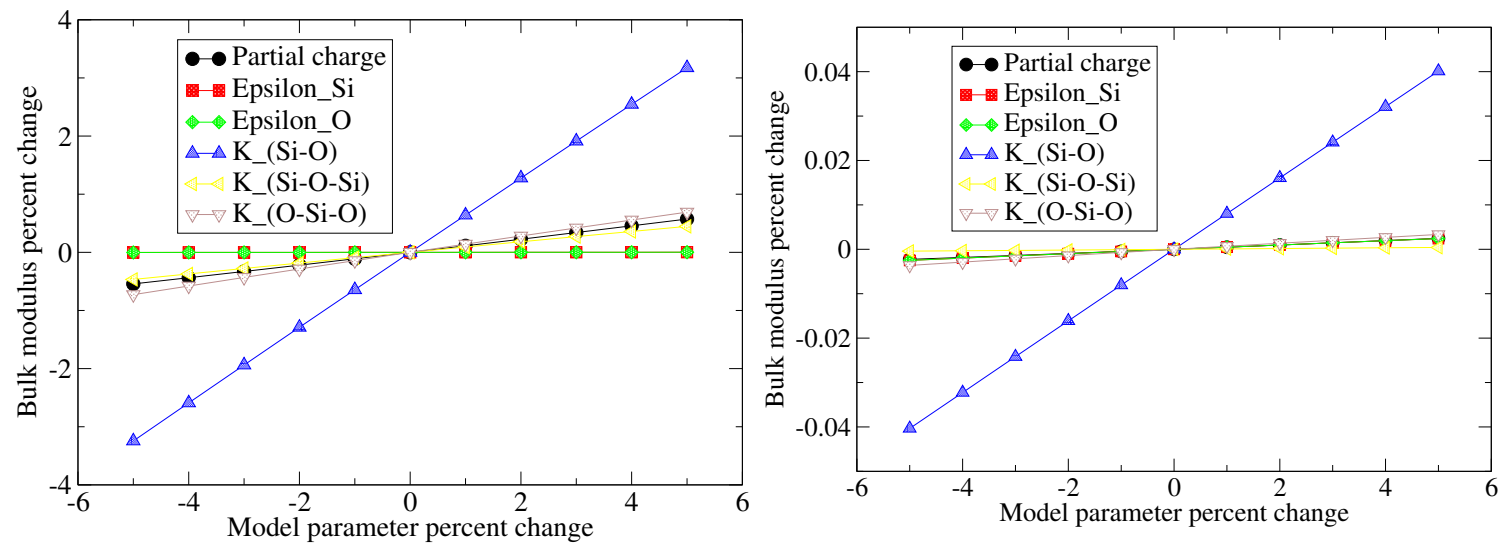

(a) Sensitivity analysis of the FAU bulk

(b) Sensitivity analysis of the SOD bulk modulus at $0 \mathrm{~K}$. modulus at $0 \mathrm{~K}$.

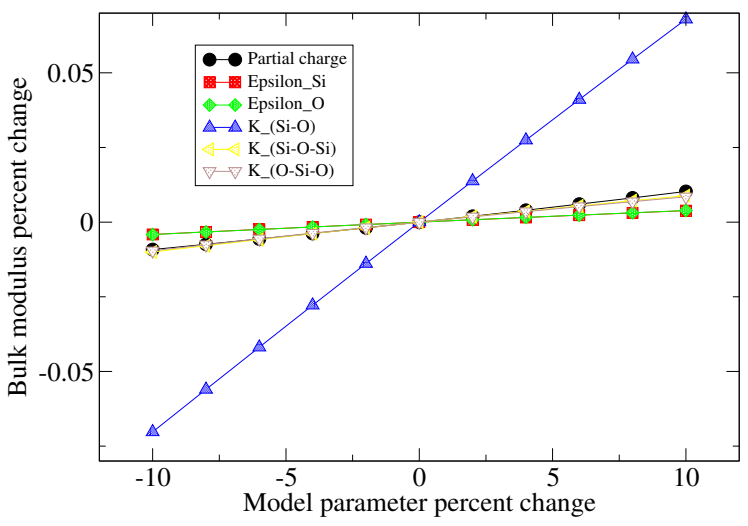

(c) Sensitivity analysis of the LTA bulk modulus at $0 \mathrm{~K}$.

Figure 4.2. Sensitivity analysis of the siliceous zeolite bulk modulus dependence on model parameters at $0 \mathrm{~K}$. Covalent interactions like $\mathrm{Si}-\mathrm{O}$ bond-stretching and $\mathrm{O}-\mathrm{Si}-\mathrm{O}$ anglebending have more significant effects on zeolite bulk moduli. 


$$
V_{\mathrm{vdW}}=\epsilon\left[\left(\frac{r_{m}}{r}\right)^{12}-2\left(\frac{r_{m}}{r}\right)^{6}\right]
$$

Note that in this new potential, the 1-4 scale method, as discussed in Section 2.1.1.2, is used. Long-range interactions between atom pairs separated by one bond and two bonds are excluded. This is because the interactions between atom pairs separated by one bond and two bonds have already been covered by bond interactions and angle interactions, respectively. Although no torsional interactions are defined in the new potential, a scaling factor of 0.68 is found by trial-and-error, and is adopted for the electrostatic interactions between atom pairs separated by three bonds in order to fit to the FAU experimental lattice parameter. The Lennard-Jones interactions between the same atom pairs are kept unchanged.

Table 4.1. Model parameters of the new potential.

\begin{tabular}{ccc}
\hline Harmonic bond potential & $k_{R}\left(\mathrm{eV} / \AA^{2}\right)$ & $R_{0}(\AA)$ \\
Si-O & 26.500 & 1.620 \\
\cline { 2 - 3 } Harmonic angle potential & $k_{\theta}\left(\mathrm{eV} / \mathrm{rad}^{2}\right)$ & $\theta_{0}\left(^{\circ}\right)$ \\
O-Si-O & 5.9000 & 109.470 \\
Si-O-Si & 0.4075 & 149.800 \\
\cline { 2 - 3 } Urey-Bradley potential & $k_{\mathrm{UB}}\left(\mathrm{eV} / \AA^{2}\right)$ & $r_{0}(\AA)$ \\
Si-O-Si & 2.3676 & 3.1261 \\
\cline { 2 - 3 } Lennard-Jones & $\epsilon(\mathrm{kcal} / \mathrm{mol})$ & $r_{m}(\AA)$ \\
$\mathrm{Si}$ & 0.6000 & 4.4 \\
O & 0.3021 & 3.54 \\
Charge & \multicolumn{2}{c}{$q(\mathrm{e})$} \\
Si/O & \multicolumn{2}{c}{$1.08 /-0.54$} \\
\hline
\end{tabular}

\subsection{Results and Discussion}

\subsubsection{Geometric Observables and Bulk Moduli at $300 \mathrm{~K}$}

Table 4.2 compares the computed geometric observables and bulk moduli of five types of silica polymorphs predicted by the original MZHB potential [21] and the 
new potential, as well as another core-shell model [22]. It is observed that the new potential predicts lattice parameters and average $\mathrm{Si}-\mathrm{O}-\mathrm{Si}$ angles for FAU, quartz, and cristobalite closer to experimentally measured values than the original MZHB potential, while the MZHB potential performs better in predicting silica polymorphs' average $\mathrm{Si}-\mathrm{O}$ bond lengths. However, only the FAU bulk modulus predicted by all three models is close to the experimental value.

\subsubsection{Infrared Spectra}

\subsubsection{FAU (Siliceous Zeolite Y)}

Figure 4.3 presents the model-predicted FAU IR spectra computed via both the original MZHB potential and the new potential, compared to a measured FAU IR spectrum [104]. As seen in Figure 4.3, the new potential reproduces roughly all the IR peaks that are observed in the measured IR spectrum, except for the shoulder at around $1200 \mathrm{~cm}^{-1}$. Note that, compared with the predictions of the original MZHB potential, the angle-bending region predicted by the new potential matches better with measured IR peaks, especially for the peak at around $420 \mathrm{~cm}^{-1}$, and the shoulder at around $493 \mathrm{~cm}^{-1}$. Recall that, based on the results of the cluster calculations in Section 3.4.2.1, one would expect that the IR peak at around $420 \mathrm{~cm}^{-1}$ and the shoulder at around $493 \mathrm{~cm}^{-1}$ predicted by the new potential are caused by the $4 \mathrm{R}$ breathing motion, and the IR peak at around $475 \mathrm{~cm}^{-1}$ caused by the $6 \mathrm{R}$ deformation

motion. Indeed, the $6 \mathrm{R}$ deformation motion is observed at around $475 \mathrm{~cm}^{-1}$; when visualized, however, the underlying molecular motions associated with the IR modes at around $420 \mathrm{~cm}^{-1}$ and $493 \mathrm{~cm}^{-1}$ do not match the cluster calculation perfectly. Another shoulder at around $775 \mathrm{~cm}^{-1}$ is also reproduced by the new potential, which is missing in the IR spectrum predicted by the original MZHB potential in Figure 3.13. Although the two external-linkage symmetric stretching modes predicted by the new potential are shifted to lower wavenumbers, we think they match with the measured 


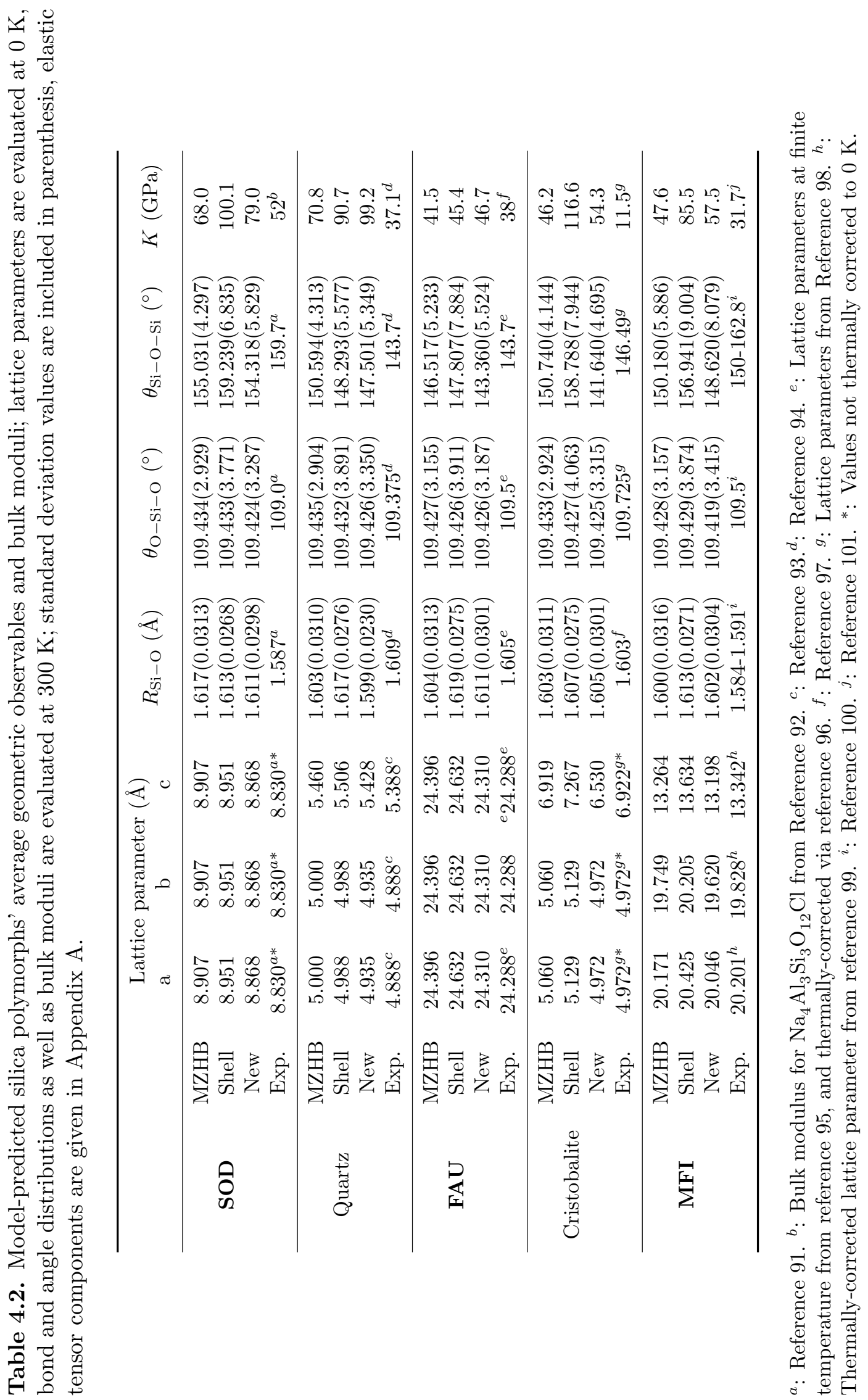




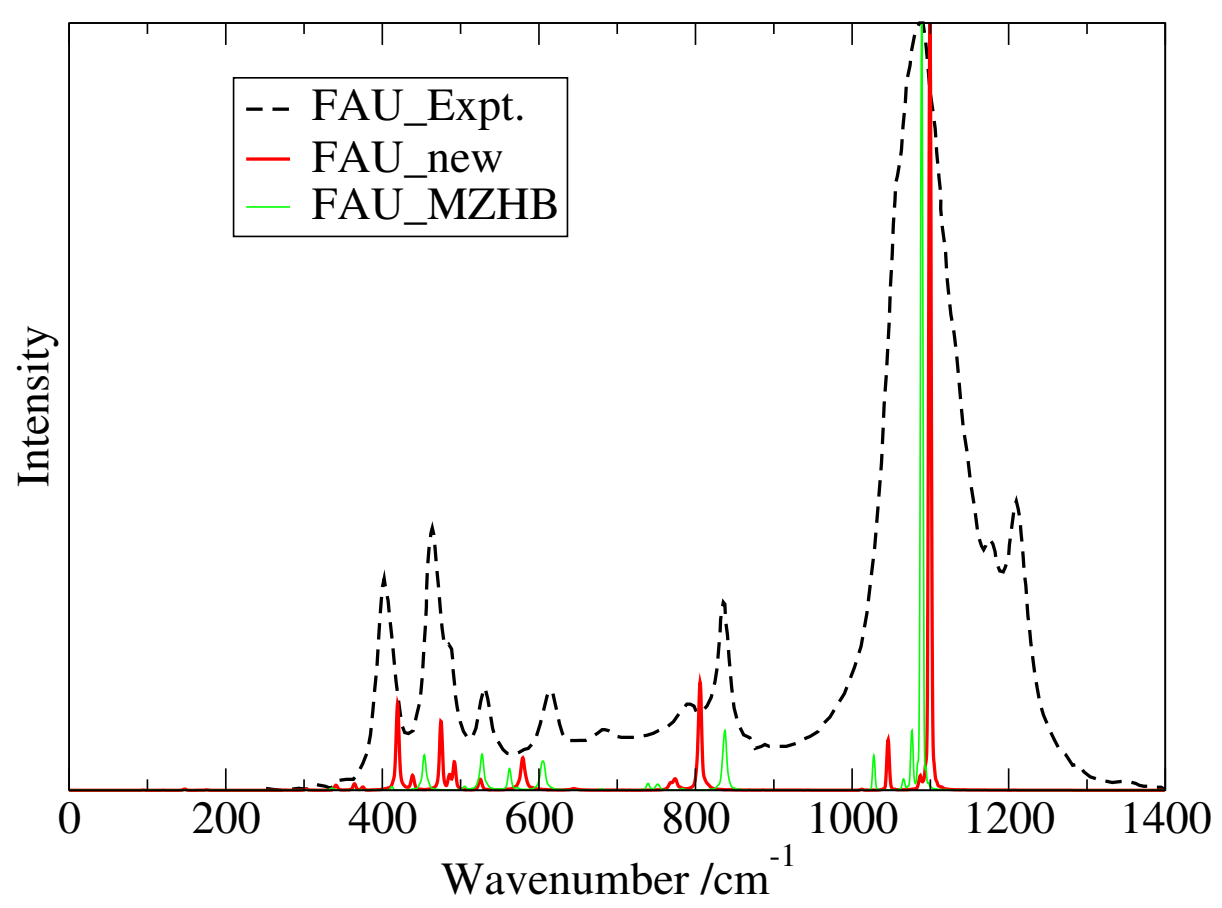

Figure 4.3. A comparison of FAU IR spectra predicted by the MZHB potential and the new potential from MD simulations at $300 \mathrm{~K}$. the IR peak at around $420 \mathrm{~cm}^{-1}$ and the shoulder at around $493 \mathrm{~cm}^{-1}$ are predicted by the new potential, while the underlying molecular motions of these two peaks are not clearly $4 \mathrm{R}$ breathing motion, as predicted by the cluster calculations in Section 3.4.2.1; the external linkage symmetric stretching region is better predicted by the new potential in the sense that a major peak accompanied by a weak shoulder is reproduced in this region. The measured spectrum is obtained from Jacobs et al. [104].

peaks better in the sense that one major peak accompanied by a weak shoulder appears in this region. A full peak assignment of the FAU IR spectrum comparing the predictions from the two models is presented in Table 4.3.

\subsubsection{SOD (siliceous sodalite)}

Figure 4.4 compares the SOD IR spectra predicted by both models with the measured IR spectrum [109]. It is clearly shown that the IR peaks predicted by the new potential are closer to the three experimentally observed IR peaks, especially for the angle-bending mode at around $450 \mathrm{~cm}^{-1}$. A full peak assignment of the SOD 
Table 4.3. Peak assignments of IR peaks predicted by the MZHB potential and the new potential, values are in $\mathrm{cm}^{-1}$, MD-calculated IR wavenumbers are calculated at $300 \mathrm{~K}$.

\begin{tabular}{|c|c|c|c|}
\hline MZHB & New & Expt. [104] & Assignment \\
\hline 453.5 & 420.5 & 418.6 & Pore opening ( $4 \mathrm{R}$ breathing $\left.(\mathrm{O}) \_\mathrm{D} 6 \mathrm{R}\right)$ \\
\hline 527.0 & 475.3 & 477.3 & Angle bending ( $6 \mathrm{R}$ deformation) \\
\hline \multirow[t]{2}{*}{561.3} & 492.7 & 491.9 & Angle bending (4R breathing $\left.(\mathrm{O}) \_\mathrm{D} 6 \mathrm{R}\right)$ \\
\hline & 525.9 & 527.6 & Angle bending (4R breathing(Si,O)_D6R) \\
\hline 605.8 & 580.3 & 580.9 & D6R block \\
\hline 837.2 & $\begin{array}{l}774.6 \\
806.8 \\
\end{array}$ & $\begin{array}{l}779.1 \\
837.2\end{array}$ & External symmetric stretching \\
\hline 1091.0 & 1099.1 & $\begin{array}{c}1099.3 \\
1210\end{array}$ & Internal asymmetric stretching \\
\hline
\end{tabular}

Table 4.4. Peak assignments of SOD IR peaks predicted by the MZHB potential and the new potential, values are in $\mathrm{cm}^{-1}$, MD-calculated IR wavenumbers are calculated at $300 \mathrm{~K}$.

\begin{tabular}{cccc}
\hline MZHB & New & Expt. [109] & Assignment \\
\hline 530.5 & 457.4 & 460 & Angle bending \\
\hline 740.3 & 754.2 & 788 & External symmetric stretching \\
795.4 & 773.1 & & Internal asymmetric stretching \\
\hline 1090.5 & 1112.6 & 1109 & \\
\hline
\end{tabular}

IR spectrum comparing the predictions of the original MZHB potential and the new potential is presented in Table 4.4 .

\subsubsection{Quartz}

Figure 4.5 presents the model-predicted quartz IR spectra together with a measured IR spectrum [110]. Note that most of the IR peaks are approximately reproduced by the new potential, except for the internal asymmetric stretching mode at around $1147 \mathrm{~cm}^{-1}$ and the shoulder at around $498 \mathrm{~cm}^{-1}$. Compared with the IR spectrum predicted by the original MZHB potential, angle-bending modes predicted 


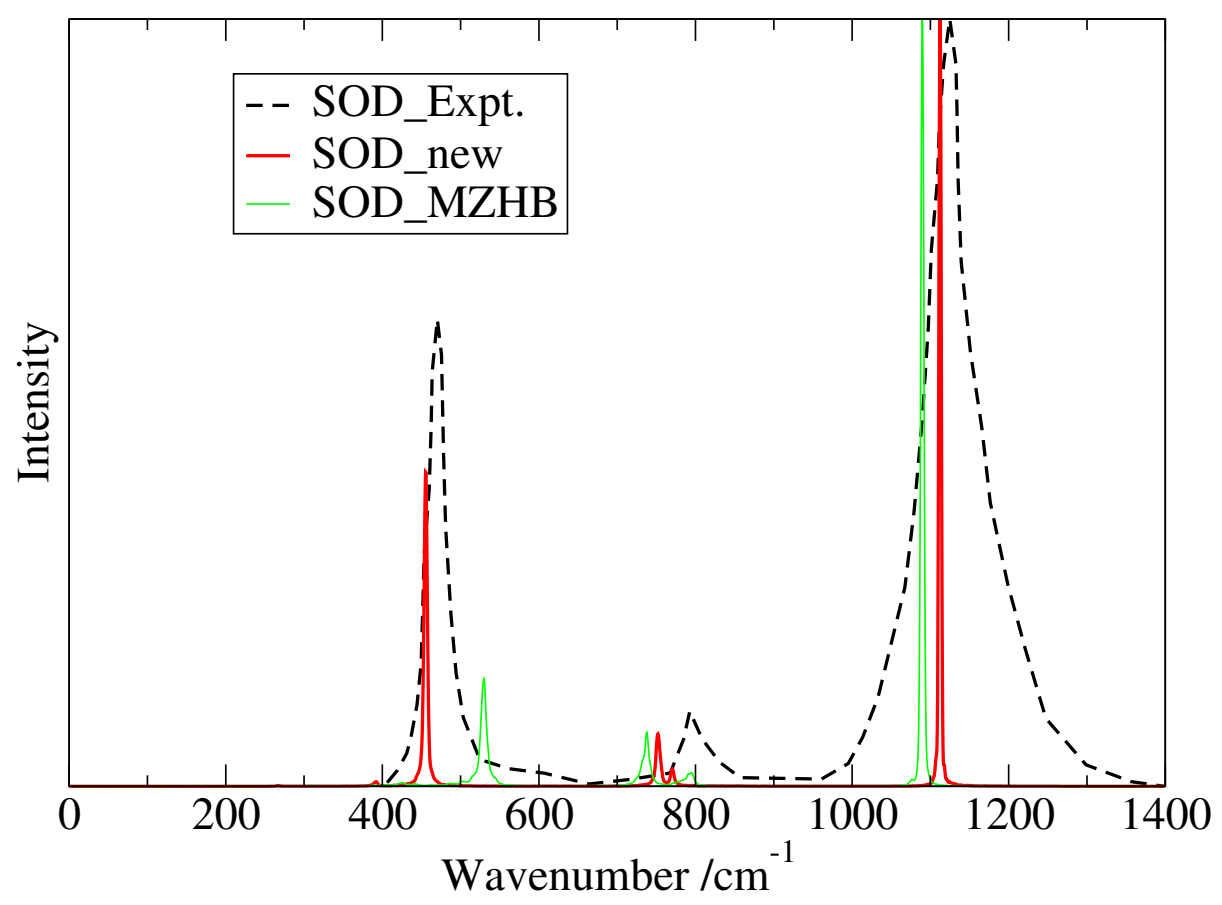

Figure 4.4. A comparison of SOD IR spectra predicted by the MZHB potential and the new potential from MD simulations at $300 \mathrm{~K}$. all the IR peaks predicted by the new potential are closer to the measured IR peaks, especially for the angle-bending mode at around $450 \mathrm{~cm}^{-1}$. The measured IR spectrum obtained from Bornhauser and Bougeard [109].

by the new potential appear at lower wavenumbers, which match with experimental peaks better. This is expected, since we conclude that the MZHB potential consistently shifts angle-bending modes to higher wavenumbers for all five types of silica polymorphs due to a large angle-bending force constant for $\mathrm{O}-\mathrm{Si}-\mathrm{O}$. The value of this parameter is intentionally reduced during the parameterization of the new potential. Another improvement is visible in the external linkage symmetric stretching region, where a clear IR peak together with a shoulder at a slightly lower wavenumber is observed. However, the major IR peak, which is the internal asymmetric stretching mode observed at around $1072 \mathrm{~cm}^{-1}$ in the measured spectrum, is shifted to a higher wavenumber, even worse than the prediction of the original MZHB potential. A full peak assignment of the quartz IR spectrum comparing the predictions from the original MZHB potential and the new potential is presented in Table 4.5. 


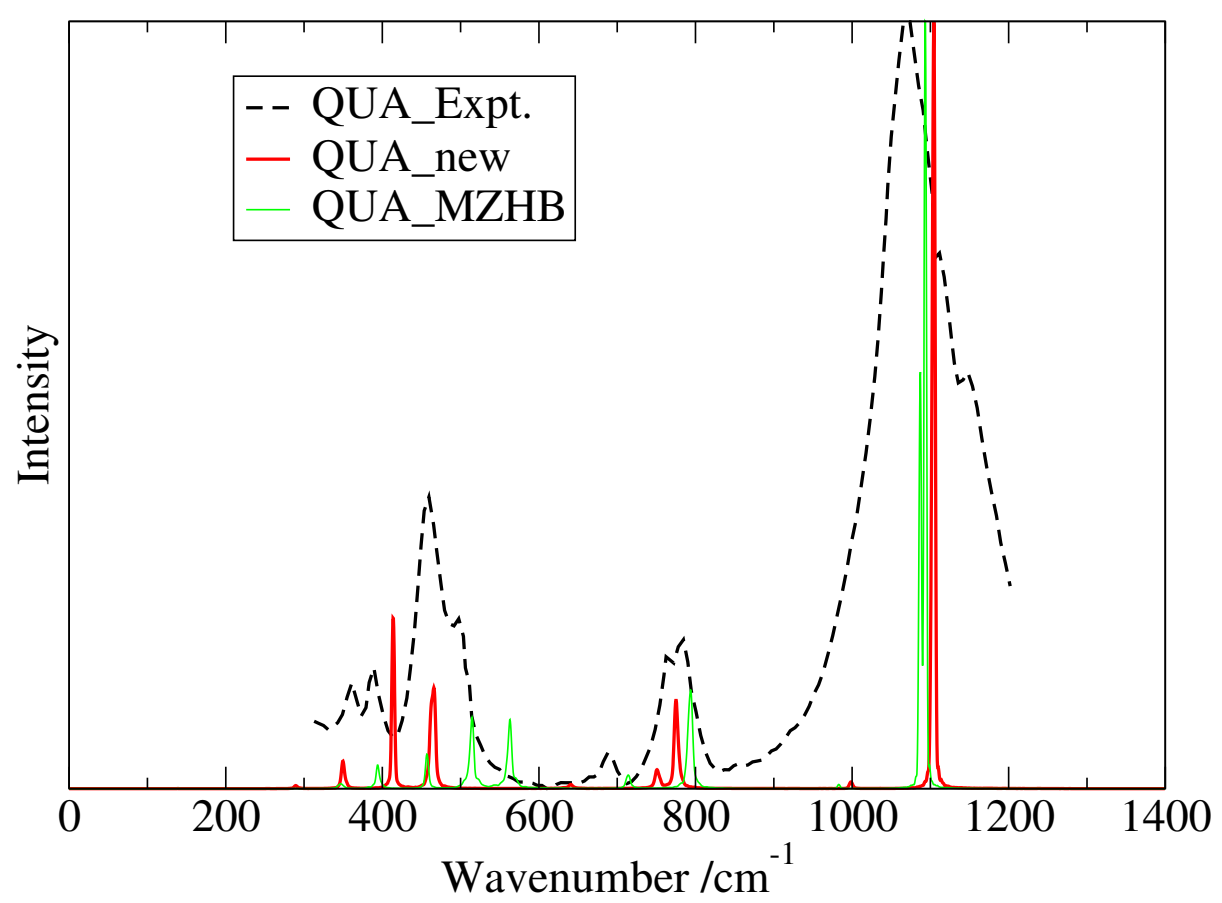

Figure 4.5. A comparison of quartz IR spectra predicted by the MZHB potential and the new potential from MD simulations at $300 \mathrm{~K}$. The angle-bending modes predicted by the new potential appears at lower wavenumbers, which match with measured peaks better compared to those predicted by the MZHB potential, except for the mismatch at around $498 \mathrm{~cm}^{-1}$; external linkage symmetric stretching peaks are also better-reproduced by the new potential in the sense that a major IR peak with a shoulder at a slightly lower wavenumber appears in this region. The measured IR spectrum is obtained from Liang et al. [110].

Table 4.5. Peak assignments of quartz IR peaks predicted by the MZHB potential and the new potential, values are in $\mathrm{cm}^{-1}, \mathrm{MD}$-calculated IR wavenumbers are calculated at $300 \mathrm{~K}$.

\begin{tabular}{cccc}
\hline MZHB & New & Expt. [110] & Assignment \\
\hline 394.6 & 350.3 & 363.0 & \\
458.3 & 414.6 & 388.8 & Angle bending \\
515.7 & 465.3 & 459.1 & \\
563.3 & & 498.6 & \\
\hline 714.1 & 640.3 & 690.7 & Internal symmetric bond stretching \\
\hline 793.7 & 751.3 & 764.5 & External symmetric stretching \\
& 774.9 & 786.8 & \\
\hline 1095.6 & 1105.3 & 1071.6 & Internal asymmetric stretching \\
& & 1147.3 & \\
\hline
\end{tabular}


Table 4.6. Peak assignments of cristobalite IR peaks predicted by the MZHB potential and the new potential, values are in $\mathrm{cm}^{-1}$, MD-calculated IR wavenumbers are calculated at $300 \mathrm{~K}$.

\begin{tabular}{|c|c|c|c|}
\hline MZHB & Modified & Expt. [110] & Assignment \\
\hline 555 & 477.9 & 472 & Angle bending \\
\hline 586 & 493.4 & 412 & \\
\hline 615 & 587.7 & 609 & Angle bending \\
\hline 771 & 791.9 & 787 & External symmetric stretching \\
\hline 1089 & 1099.1 & 1086 & Internal asymmetric bond stretching \\
\hline
\end{tabular}

\subsubsection{Cristobalite}

Comparison of model-predicted cristobalite IR spectra by both the MZHB potential and the new potential with the measured spectrum [110] is presented in Figure 4.6. It is observed that while the measured angle-bending mode at around $472 \mathrm{~cm}^{-1}$ is predicted at a higher wavenumber above $550 \mathrm{~cm}^{-1}$ by the original MZHB potential, this peak is well-reproduced by the new potential at around $478 \mathrm{~cm}^{-1}$. Improvement of the prediction is also observed at the external linkage symmetric stretching region, where a good match between the model prediction and experimental data is achieved at around $790 \mathrm{~cm}^{-1}$. However, the measured IR peak at around $609 \mathrm{~cm}^{-1}$ is better-reproduced by the MZHB potential. A full peak assignment of the cristobalite IR spectrum comparing the predictions of the original MZHB potential and the new potential is presented in Table 4.6.

\subsubsection{MFI (silicalite-1)}

Figure 4.7 presents the comparison of MFI IR spectra predicted by both the MZHB potential and the new potential with the measured spectrum [109]. It is clear that in the measured IR spectrum, two broad angle-bending modes are observed at 400-500 $\mathrm{cm}^{-1}$ and 500-600 $\mathrm{cm}^{-1}$, respectively. However, the MZHB potential only predicts one broad band at $400-600 \mathrm{~cm}^{-1}$. On the other hand, the new potential 


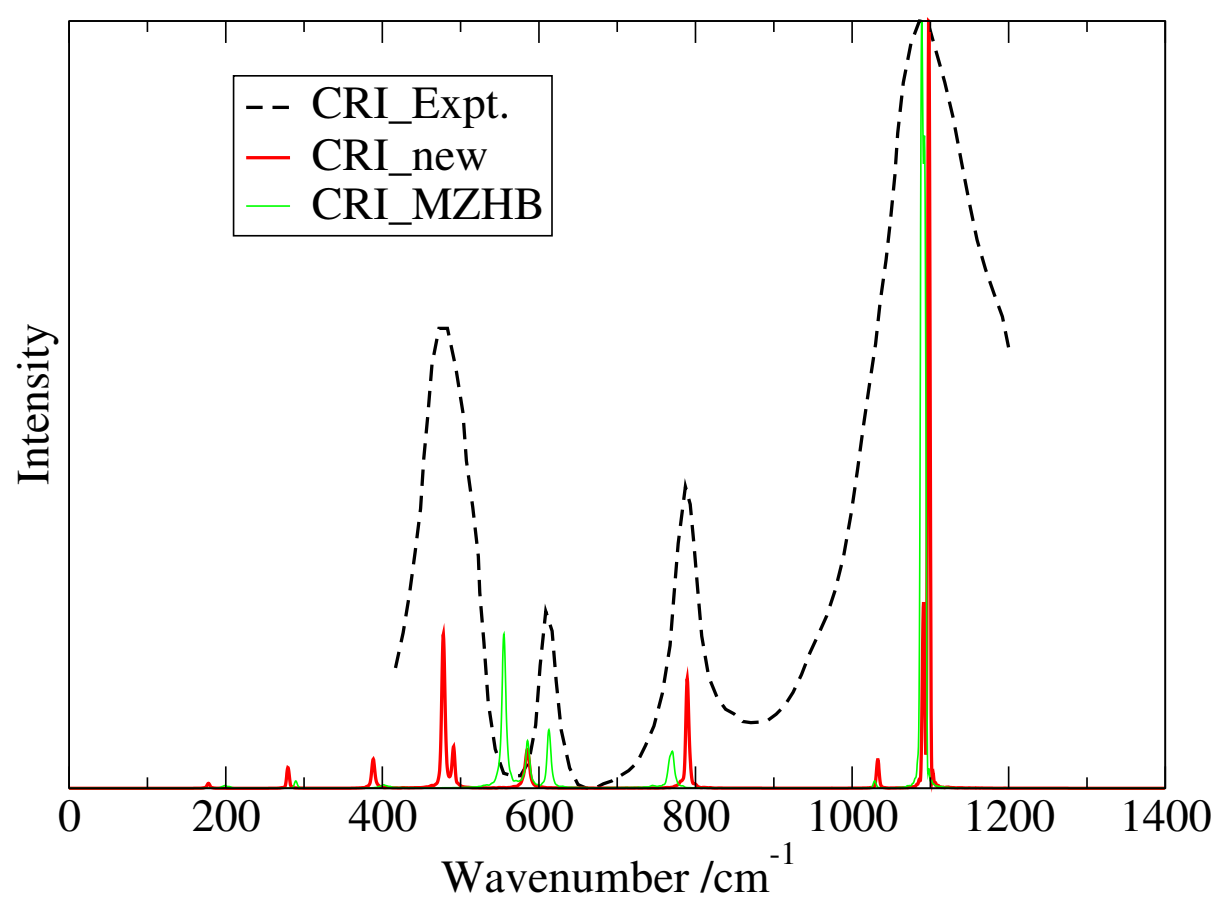

Figure 4.6. Comparison of cristobalite IR spectra predicted by the MZHB potential and the new potential from MD simulations at $300 \mathrm{~K}$. While the MZHB potential shifts the angle-bending modes to higher wavenumbers, the new potential better predicts this mode at around $478 \mathrm{~cm}^{-1}$; better prediction by the new potential is also observed in the external linkage symmetric stretching region at around $800 \mathrm{~cm}^{-1}$. The measured IR spectrum is obtained from Liang et al. [110].

clearly predicts two separated angle-bending modes at $400-500 \mathrm{~cm}^{-1}$ and around $520 \mathrm{~cm}^{-1}$, respectively, with slight shifts compared to the measured spectrum. The external linkage symmetric stretching mode predicted at around $780 \mathrm{~cm}^{-1}$ by the new potential also matches better with the measured peak observed at around $800 \mathrm{~cm}^{-1}$.

\subsection{Conclusion}

In this chapter, a sensitivity analysis is performed in order to study the dependence of siliceous zeolite lattice parameter and bulk modulus at $0 \mathrm{~K}$ on the MZHB potential parameters published by Sahoo and Nair [21]. The sensitivity analysis on the lattice parameter shows that atomic partial charges of silicon and oxygen atoms 


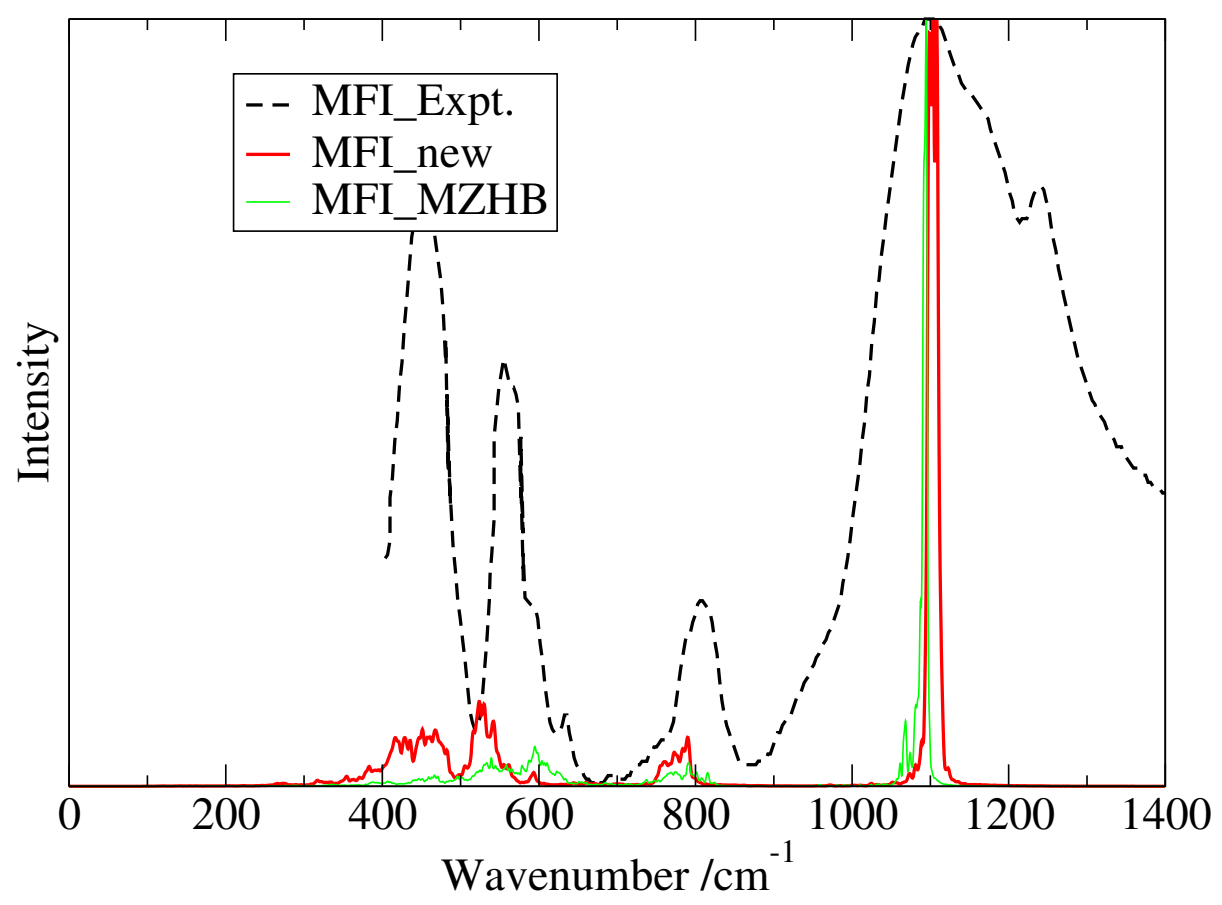

Figure 4.7. A comparison of MFI IR spectra predicted by the MZHB potential and the new potential from MD simulations at $300 \mathrm{~K}$. While the MZHB potential predicts only one broad angle-bending mode in the region from $400-600 \mathrm{~cm}^{-1}$, the new potential clearly predicts two separated modes at $400-500 \mathrm{~cm}^{-1}$ and around $520 \mathrm{~cm}^{-1}$, respectively; better prediction of the external linkage symmetric stretching mode at around $800 \mathrm{~cm}^{-1}$ is also observed. The measured IR spectrum is obtained from Serrano et al. [111].

have the most significant effect on the lattice parameter, indicating a possible new target for the parameterization of atomic partial charges of crystalline materials. On the basis of the MZHB potential, a new potential has been developed by fitting to the measured FAU IR spectrum, lattice parameter, and bulk modulus. Comparison of the performance of both the original MZHB potential and the new potential shows that although the new potential does not achieve significant improvements in the prediction of silica polymorphs' geometries, the ability of the new potential in the prediction of silica polymorphs' IR spectra is better than that of the original MZHB potential. We observe that the MZHB potential consistently shifts angle-bending modes to higher wavenumbers, while the predictions by the new potential match with measured IR spectra better for all five types of silica polymorphs studied in this 
work. While the most significant improvement is seen in the angle-bending region from 400-600 $\mathrm{cm}^{-1}$, improvement in the external linkage symmetric stretching region at around $800 \mathrm{~cm}^{-1}$ is also observed. These improvements in the prediction of these silica polymorphs' IR spectra indicate that the new potential reflects the siliceous zeolite framework interactions better than the original MZHB potential. The use of the CHARMM-derived Lennard-Jones parameters in the newly developed potential and the transposition error in the MZHB potential also make the new potential preferable when applied in computational modeling of zeolite application processes, such as catalytic chemical reactions and adsorption, since the Lennard-Jones interaction is one of the dominating host-guest (zeolite framework-reacting species or adsorbate) interactions in these processes. 


\section{CHAPTER 5}

\section{CONCLUSION}

The present work can be divided roughly into two parts. In the first part, two

published potentials, the MZHB potential and the core-shell model, are evaluated in terms of their ability to predict the equilibrium geometry, bulk modulus, and IR spectrum of siliceous zeolites. Comparisons between model predictions and experimental data show that the MZHB potential is better at reproducing silica polymorphs' geometries, while the core-shell model predicts broader angle distributions and larger average bond angles; we think this happens because the core-shell model lacks three-body interactions that constrain the $\mathrm{Si}-\mathrm{O}-\mathrm{Si}$ angles. This inherent defect of the core-shell model is also confirmed by model-predicted IR spectra for silica polymorphs in the sense that the angle-bending modes predicted by the core-shell model are shifted to lower wavenumbers. On the other hand, the discussion based on normal mode visualizations of the FAU IR peaks predicted by the MZHB potential and the cluster calculation has been given in detail to demonstrate that the MZHB potential consistently shifts angle-bending modes to higher wavenumbers. A model-computed IR spectrum is characteristic of the potential energy surface of the material; from this perspective, these inconsistencies between model-predicted IR spectra and experimentally observed IR spectra indicate that these two potentials are less accurate to describe the interactions of zeolite frameworks.

The second part of this work focused on the development and testing of a new potential via reparameterizing and extending the MZHB potential. A sensitivity 
analysis was first performed to investigate the dependencies of the siliceous zeolite lattice parameter and bulk modulus on model parameters. We observed that while the bulk modulus is very sensitive to bond and angle force constants, the lattice parameter is most sensitive to atomic partial charges, indicating a possible new target for the parameterization of atomic partial charges of crystalline materials. Then, following the sensitivity analysis, parametrization of the new potential is carried out by fitting to the experimental FAU lattice parameter and bulk modulus. We observed that the new potential predicts IR peaks for silica polymorphs more accurately than the MZHB and core-shell model. The improvement in the prediction of IR spectra indicates that this newly-developed potential does a better job of describing zeolite framework interactions than its predecessor. It also means that the new potential will probably be a better model for the modeling of mass and heat transfer processes in zeolite applications.

Future work will include further refinements of the new potential to be a general model for the modeling of various zeolites' IR spectra; such a general model can also help discover possible new zeolites by comparing the model-predicted IR spectra with existing zeolite database. The existence of chemically-adsorbed species or reaction intermediates will often change the pattern of the host's IR spectrum, due to the change of the of chemical environment and the additional vibrational modes of the guest species; these changes in IR spectra are often helpful in identifying different adsorbates and reaction intermediates. A good model of zeolite frameworks and hostguest interactions can greatly help examine the experimentally observed adsorbates and intermediates via computational studies. In the present work, the new potential has demonstrated its ability to predicts IR spectra of zeolite frameworks, therefore, future work will also include incorporating aluminum atoms, charge-compensating cations, and guest species in the newly-developed potential. We expect that such a 
complete potential will contribute to the theoretical studies of catalyst characterization, as well as to studies of catalytic reactions and adsorption. 


\section{APPENDIX A \\ MD-PREDICTED UNIQUE ELASTIC TENSOR COMPONENTS AT $300 \mathrm{~K}$}

The unique elastic tensor components of silica polymorphs are calculated following the procedure described in Section 3.2. Formulas used for the calculation of bulk modulus $(K)$ and shear modulus $(G)$ are given below corresponding tables.

Table A.1. MD-predicted FAU elastic tensor components, bulk modulus $K$, and shear modulus $G$ at $300 \mathrm{~K}$, in GPa. The crystal structure is cubic, so $C_{11}, C_{22}$, and $C_{44}$ are the only unique components of the stiffness tensor.

\begin{tabular}{cccccc}
\hline & $C_{11}$ & $C_{12}$ & $C_{44}$ & $K$ & $G$ \\
\cline { 2 - 6 } MZHB & 77.76 & 23.39 & 24.62 & 41.51 & 25.65 \\
Shell & 85.91 & 25.08 & 26.34 & 45.36 & 27.97 \\
New & 82.05 & 28.96 & 25.59 & 46.66 & 25.97 \\
\hline
\end{tabular}

$K=\frac{1}{3}\left(C_{11}+2 C_{12}\right) ; G=\frac{1}{5}\left(C_{11}+3 C_{44}-C_{12}\right)$ 
Table A.2. MD-predicted SOD elastic tensor components, bulk modulus $K$, and shear modulus $G$ at $300 \mathrm{~K}$, in GPa. The crystal structure is cubic, so $C_{11}, C_{22}$, and $C_{44}$ are the only unique components of the stiffness tensor.

\begin{tabular}{cccccc}
\hline & $C_{11}$ & $C_{12}$ & $C_{44}$ & $K$ & $G$ \\
\cline { 2 - 5 } MZHB & 122.83 & 40.55 & 36.89 & 67.98 & 38.59 \\
shell & 134.42 & 83.00 & 26.12 & 100.14 & 25.96 \\
New & 131.73 & 52.66 & 33.36 & 79.02 & 35.83 \\
\hline
\end{tabular}

$K=\frac{1}{3}\left(C_{11}+2 C_{12}\right) ; G=\frac{1}{5}\left(C_{11}+3 C_{44}-C_{12}\right)$

Table A.3. MD-predicted quartz elastic tensor components, bulk modulus $K$ and shear modulus $G$ at $300 \mathrm{~K}$, in GPa. The crystal structure is trigonal.

\begin{tabular}{cccccccccccc}
\hline & $C_{11}$ & $C_{33}$ & $C_{12}$ & $C_{13}$ & $C_{14}$ & $C_{24}$ & $C_{44}$ & $C_{56}$ & $C_{66}$ & $K$ & $G$ \\
\cline { 2 - 11 } MZHB & 148.67 & 137.77 & 39.58 & 39.29 & -3.33 & 7.54 & 60.85 & -6.59 & 55.31 & 74.6 & 56.5 \\
shell & 139.97 & 156.09 & 37.04 & 62.07 & -8.17 & -0.13 & 52.63 & -6.54 & 51.79 & 84.3 & 49.7 \\
New & 175.21 & 160.01 & 56.90 & 64.18 & 0.12 & -0.52 & 74.27 & 0.63 & 57.82 & 91.5 & 63.0 \\
\hline
\end{tabular}

$K=\frac{1}{9}\left(2 C_{11}+C_{33}+2\left(C_{12}+2 C_{13}\right)\right)$

$G=\frac{1}{15}\left(2 C_{11}+C_{33}+3\left(2 C_{44}+C_{66}\right)-C_{12}-C_{13}\right)$

Table A.4. MD-predicted cristobalite elastic tensor components, bulk modulus $K$, and shear modulus $G$ at $300 \mathrm{~K}$, in GPa. The crystal structure is tetragonal; only unique tensor components are listed.

\begin{tabular}{ccccccccc}
\hline & $C_{11}$ & $C_{33}$ & $C_{12}$ & $C_{13}$ & $C_{44}$ & $C_{66}$ & $K$ & $G$ \\
\cline { 2 - 8 } MZHB & 129.89 & 83.38 & 12.30 & 12.00 & 60.22 & 44.78 & 46.20 & 53.50 \\
Shell & 159.21 & 156.05 & 98.91 & 94.24 & 39.47 & 23.62 & 116.58 & 32.98 \\
New & 169.37 & 69.43 & 14.44 & 13.00 & 65.86 & 40.15 & 54.34 & 58.89 \\
\hline
\end{tabular}

$K=\frac{1}{9}\left(2 C_{11}+C_{33}+2\left(C_{12}+2 C_{13}\right)\right)$

$G=\frac{1}{5}\left(2 C_{11}+C_{33}+3\left(2 C_{44}+C_{66}\right)-C_{12}-2 C_{23}\right)$ 
Table A.5. MD-predicted MFI elastic tensor components, bulk modulus $K$, and shear modulus $G$ at $300 \mathrm{~K}$ in GPa, the crystal structure is orthorhombic.

\begin{tabular}{cccccccccccc}
\hline & $C_{11}$ & $C_{22}$ & $C_{33}$ & $C_{12}$ & $C_{13}$ & $C_{23}$ & $C_{44}$ & $C_{55}$ & $C_{66}$ & $K$ & $G$ \\
\cline { 2 - 11 } MZHB & 114.62 & 106.11 & 91.52 & 17.56 & 23.47 & 17.05 & 38.99 & 40.15 & 35.52 & 47.59 & 39.88 \\
Shell & 124.90 & 130.13 & 136.31 & 55.30 & 66.84 & 66.90 & 30.73 & 28.80 & 23.98 & 85.49 & 30.19 \\
New & 129.46 & 114.02 & 106.29 & 27.01 & 34.44 & 22.32 & 35.41 & 41.11 & 32.38 & 57.48 & 39.51 \\
\hline
\end{tabular}

$K=\frac{1}{9}\left(C_{11}+C_{22}+C_{33}+2\left(C_{12}+C_{13}+C_{23}\right)\right) ;$

$G=\frac{1}{15}\left(C_{11}+C_{22}+C_{33}+3\left(C_{44}+C_{55}+C_{66}\right)-C_{12}-C_{13}-C_{23}\right)$ 


\section{APPENDIX B}

\section{IR SPECTRA OF QUANTUM MECHANICAL CLUSTERS}

In order to investigate the characteristic vibrational modes of FAU and their dependence on the size of the cluster, a series of quantum mechanical calculations were performed on clusters characterize the structure of FAU. As presented in Figures B.1a, B.1b and B.1c, the structure of these three clusters are built up by surrounding a double 6-membered ring (D6R) with three, four, and five layers of atoms, respectively. Calculations performed on these three clusters can help identify characteristic vibrational modes that consistently exist, regardless the size of the clusters. As the size of the cluster gets bigger and bigger, the properties of the cluster will approach to the properties of the periodic FAU structure. Thus, one can expect that these characteristic vibrational modes also exist in the periodic FAU structure. Figures B.1d and B.1e show the other two clusters used in this work, which are a sodalite cage and a sodalite cage covered by three layers of atoms. Calculations performed on these two clusters can help identify the origins of certain IR modes.

All clusters were terminated by hydrogen atoms, whose positions were determined following the procedure described in the work of Hammond [116], Section 6.4. Specifically, to construct each cluster, a two-layer GAUSSIAN ONIOM [106] calculation was performed first on the periodic FAU structure with the debugging keyword iop $(1 / 33=1)$, positions of the linker hydrogen atoms could then be grabbed from the output. The higher layer model was then taken as the cluster model terminated with those linker hydrogen atoms. Calculations were performed at HF/6-31G* with 


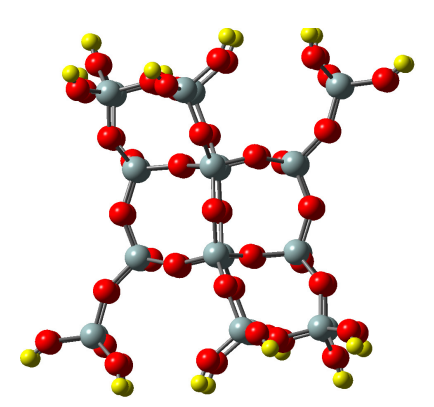

(a) D6R+3layer

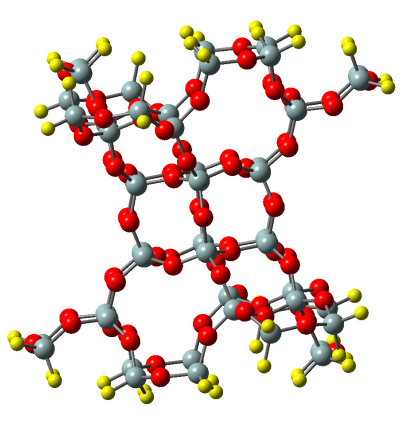

(b) D6R+4layer

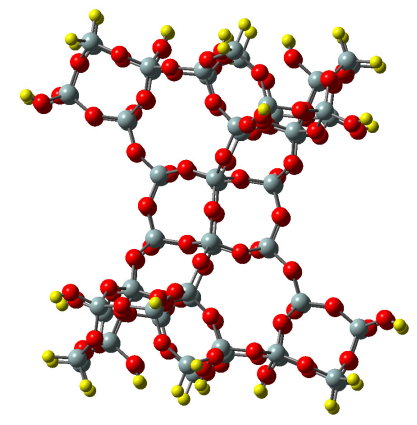

(c) D6R+5layer

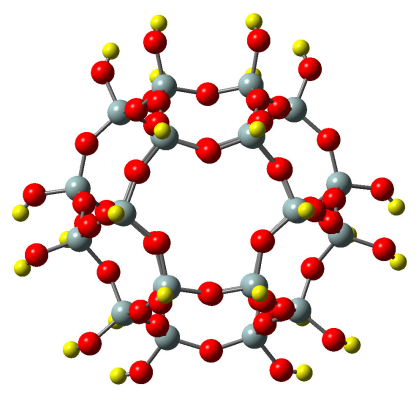

(d) Sodalite cage

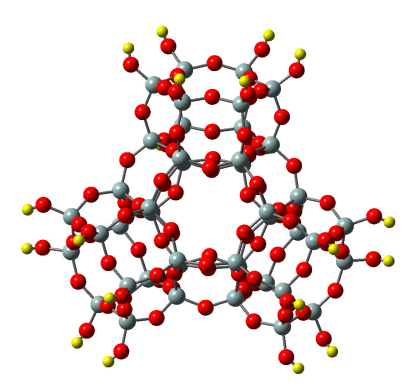

(e) Sodalite cage+3layer

Figure B.1. Cluster models used in quantum mechanical calculations.

the terminal hydrogen atoms and the atoms connected to the hydrogen atoms frozen. The resulting IR spectra are presented in Figure B.2. The vibrational frequencies are scaled by a factor of 0.8953 , as per the work of Scott and Radom [107]. We observe that, although the IR spectra of different clusters vary in both peak wavenumbers and intensities due to different degrees of freedom and boundary effects imposed by the frozen terminal groups, the relative positions of some vibrational modes are consistent regardless of the size of the clusters. Specifically, two vibrational modes associated with the 4-membered ring (4R) breathing motion consistently appear in the IR spectra of the four cluster models that contain at least one complete D6R block; and one vibrational mode associated with the 6 -membered ring (6R) deformation consistently exists in the IR spectra of all five cluster models; the two 4R breathing modes appear on both sides of the $6 \mathrm{R}$ deformation mode. The above observations indicate that the 


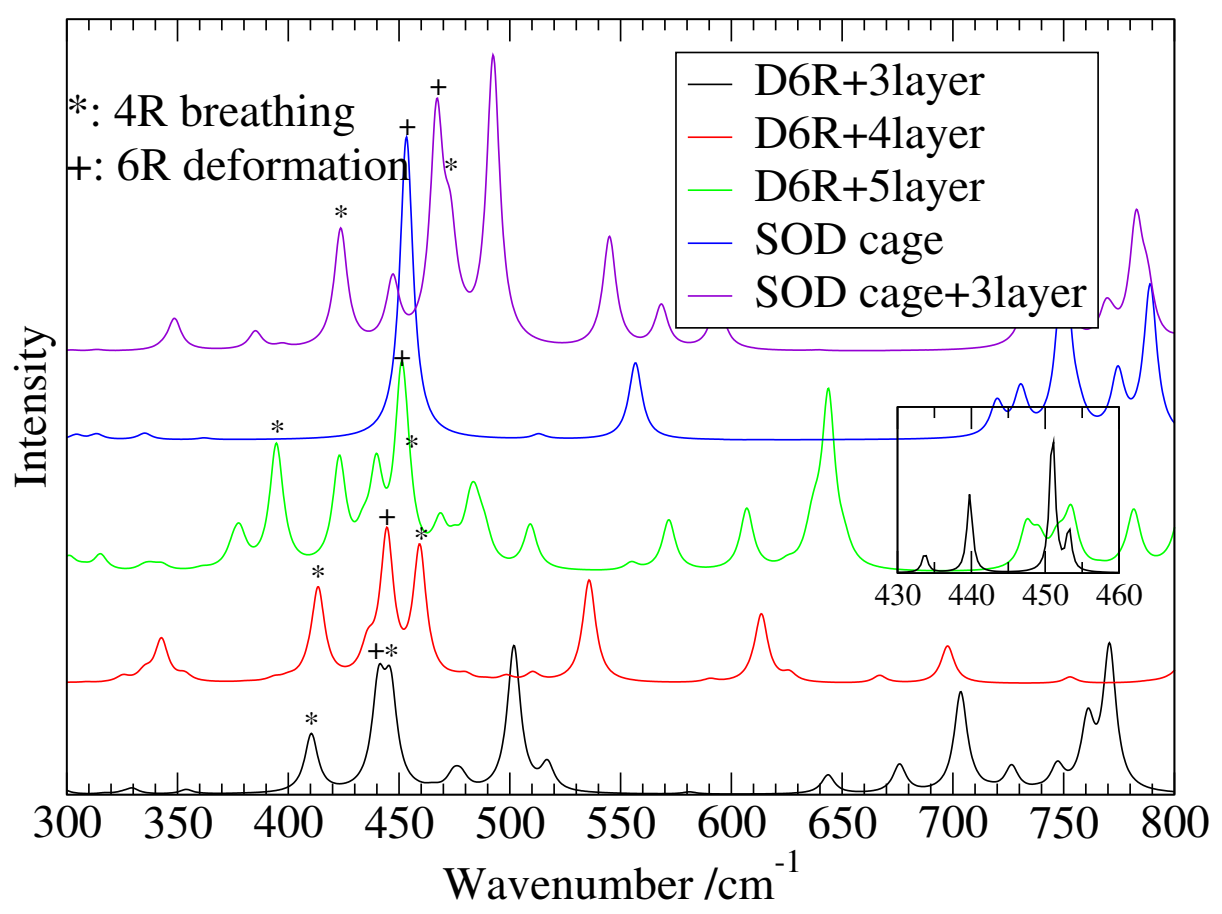

Figure B.2. IR spectra of clusters used in this work with a spectral resolution of 0.41 $\mathrm{cm}^{-1}$ : two 4R breathing modes are consistently observed in the IR spectra of four clusters and a $6 \mathrm{R}$ deformation mode is consistently observed in the IR spectra of all the five clusters, indicating that the two $4 \mathrm{R}$ breathing modes and the $6 \mathrm{R}$ deformation mode are characteristic vibrational modes of FAU structure; one can expect that these three vibrational modes also exist in the periodic FAU structure. Comparison between the IR spectrum of the sodalite cage with the other four spectra shows that while the $6 \mathrm{R}$ deformation mode originates from the vibrations of the sodalite cage, the two $4 \mathrm{R}$ breathing modes originate from the vibrations the D6R blocks. Note that the $4 \mathrm{R}$ breathing mode at around $510 \mathrm{~cm}^{-1}$ is hard to identify because of the spectral broadening effect, however, this mode is clear when viewed from GAUSSIAN normal model analysis (see the inset).

two $4 \mathrm{R}$ breathing modes and the $6 \mathrm{R}$ deformation mode are characteristic vibrational modes of FAU structure, one can expect that these three vibrational modes also exist in the periodic FAU structure; while the $6 \mathrm{R}$ deformation mode originates from the vibrations of the sodalite cage, the two $4 \mathrm{R}$ breathing modes originate from the vibrations of D6R blocks. 


\section{BIBLIOGRAPHY}

[1] Baerlocher, C.; McCusker, L. Database of Zeolite Structures. http://www . iza-structure.org/databases/.

[2] Extruded NaX Synthetic Zeolite. https://en.wikipedia.org/wiki/Zeolite, 2017.

[3] Natural Zeolite Ca-faujasite. http://www.zeolite-collection.eu/ occurrences/Europe/Deutschland/Kaiserstuhl/Limberg/Faujasite. html, 2017.

[4] Natural zeolite chabazite. http://www.mineralien-verkauf .de/zeogallery/ zeolithee.html, 2017.

[5] Wireframe of Zeolite Sodalite (SOD). http://europe.iza-structure.org/ IZA-SC/framework $\cdot$ php?STC=SOD, 2017.

[6] Wireframe of Zeolite Faujasite (FAU). http://europe.iza-structure.org/ IZA-SC/framework . php?STC=FAU, 2017.

[7] Farnworth, K. J.; O’Malley, P. J. Brønsted Acid Site Models for Zeolites: A Comparative Density Functional, Hartree-Fock and Semi-empirical Molecular Orbital Study. Electronic Journal of Theoretical Chemistry 1996, 1, 172-182.

[8] Migliori, M.; Aloise, A.; Giordano, G. Methanol to Dimethylether on H-MFI Catalyst: The Influence of the Si/Al Ratio on Kinetic Parameters. Catalysis Today 2014, 227, 138-143.

[9] Bhatia, S. Zeolite Catalysts: Principles and Applications; CRC Press: Boca Raton, Florida, 1989.

[10] Ashraf, M. T.; Chebbi, R.; Darwish, N. A. Process of p-Xylene Production by Highly Selective Methylation of Toluene. Industrial \& Engineering Chemistry Research 2013, 52, 13730-13737.

[11] Sherry, H. S.; Walton, H. F. The Ion-exchange Properties of Zeolites. II. Ion Exchange in the Synthetic Zeolite Linde 4A. Journal of Physical Chemistry 1967, 71, 1457-1465. 
[12] Yousef, R. I.; El-Eswed, B.; Al-Muhtaseb, A. H. Adsorption Characteristics of Natural Zeolites as Solid Adsorbents for Phenol Removal From Aqueous Solutions: Kinetics, Mechanism, and Thermodynamics Studies. Chemical Engineering Journal 2011, 171, 1143-1149.

[13] Galloway, F.; Ghosh, A.; Shafiei, M.; Loezos, P. Aromatic Alkylation Process. U.S. Patent WO2010138504 A1, 2010.

[14] Beauvais, C.; Boutin, A.; Fuchs, A. H. Adsorption of Water in Zeolite Sodiumfaujasite: A Molecular Simulation Study. Comptes Rendus Chimie 2005, 8, 485-490.

[15] Demir, B.; Ahunbay, M. G. Prediction of Perfluorohexane Adsorption in BCR704 Zeolite via Molecular Simulation. Fluid Phase Equilibria 2014, 366, 152158.

[16] Subbotin, A. N.; Zhidomirov, G. M.; Subbotina, I. R.; Kazansky, V. B. Molecular and Dissociative Adsorption of $\mathrm{H}_{2} \mathrm{O}$ on Zeolite Zn/ZSM-5 Studied by Diffusereflectance IR Spectroscopy and Quantum Chemical Calculations. Kinetics and Catalysis 2013, 54, 744-748.

[17] Thomas, M.; Brehm, M.; Fligg, R.; Vohringer, P.; Kirchner, B. Computing Vibrational Spectra from ab initio Molecular Dynamics. Physical Chemistry Chemical Physics 2013, 15, 6608-6622.

[18] Ermoshin, V. A.; Smimov, K. S.; Bougeard, D. Ab Initio Generalized Valence Force Field for Zeolite Modelling. 1. Siliceous Zeolites. Chemical Physics 1996, 202, 53-61.

[19] Smirnov, K. S.; Bougeard, D. Molecular Dynamics Study of the Vibrational Spectra of Siliceous Zeolites Built from Sodalite Cages. Journal of Physical Chemistry 1993, 97, 9434-9440.

[20] Nicholas, J. B.; Hopfinger, A. J.; Trouw, F. R.; Iton, L. E. Molecular Modeling of Zeolite Structure. 2. Structure and Dynamics of Silica Sodalite and Silicate Force Field. Journal of the American Chemical Society 1991, 113, 4792-4800.

[21] Sahoo, S. K.; Nair, N. N. A Potential with Low Point Charges for Pure Siliceous Zeolites. Journal of Computational Chemistry 2015, 36, 1562-1567.

[22] Schröder, K.-P.; Sauer, J. Potential Functions for Silica and Zeolite Catalysts Based on ab Initio Calculations. 3. A Shell Model Ion Pair Potential for Silica and Aluminosilicates. Journal of Physical Chemistry 1996, 100, 11043-11049.

[23] Hill, J. R.; Sauer, J. Molecular Mechanics Potential for Silica and Zeolite Catalysts Based on ab initio Calculations. 1. Dense and Microporous Silica. Journal of Physical Chemistry 1994, 98, 1238-1244. 
[24] Hopfinger, A. J.; Pearlstein, R. A. Molecular Mechanics Force-Field Parameterization Procedures. Journal of Computational Chemistry 1984, 5, 486-499.

[25] Mishin, Y.; Mehl, M.; Papaconstantopoulos, D. Phase Stability in the Fe-Ni System: Investigation by First-principles Calculations and Atomistic Simulations. Acta Materialia 2005, 53, 4029-4041.

[26] Kukol, A. Molecular Modeling of Proteins; Humana Press, 2008; Chapter 4.

[27] Zimmerman, P. M.; Head-Gordon, M.; Bell, A. T. Selection and Validation of Charge and Lennard-Jones Parameters for QM/MM Simulations of Hydrocarbon Interactions with Zeolites. Journal of Chemical Theory and Computation 2011, 7, 1695-1703.

[28] Plimpton, S. Fast Parallel Algorithms for Short-range Molecular Dynamics. Journal of Computational Physics 1995, 117, 1-19.

[29] Gale, J. D.; Rohl, A. L. The General Utility Lattice Program (GULP). Molecular Simulation 2003, 29, 291-341.

[30] Brooks, B. R.; Brooks, C. L., III; MacKerell, A. D., Jr.; Nilsson, L.; Petrella, R. J.; Roux, B.; Won, Y.; Archontis, G.; Bartels, C.; Boresch, S.; Caflisch, A.; Caves, L.; Dinner, Q. C. A. R.; Feig, M.; Fischer, S.; Gao, J.; Hodoscek, M.; Im, W.; Kuczera, K.; Lazaridis, T.; Ma, J.; Ovchinnikov, V.; Paci, E.; Pastor, R. W.; Post, C. B.; Pu, J. Z.; Schaeer, M.; Tidor, B.; Vnable, R. M.; Woodcock, H. L.; Wu, X.; Yang, W.; York, D. M.; Karplus, M. CHARMM: The Biomolecular Simulation Program. Journal of Computational Chemistry 2009, 30, 1545-1614.

[31] Mayne, C. G.; Saam, J.; Schulten, K.; Tajkhorshid, E.; Gumbart, J. C. Rapid Parameterization of Small Molecules Using the Force Field Toolkit. Journal of Computational Chemistry 2013, 34, 2757-2770.

[32] Lopes, P. E. M.; Murashov, V.; Tazi, M.; Demchuk, E.; MacKerell, A. D. Development of an Empirical Force Field for Silica. Application to the Quartz-Water Interface. Journal of Physical Chemistry B 2006, 110, 2782-2792.

[33] Gabrieli, A.; Sant, M.; Demontis, P.; Suffritti, G. B. Development and Optimization of a New Force Field for Flexible Aluminosilicates, Enabling Fast Molecular Dynamics Simulations on Parallel Architectures. Journal of Physical Chemistry C 2013, 117, 503-509.

[34] Wang, J.; Wolf, R. M.; Caldwell, J. W.; Kollman, P. A.; Case, D. A. Development and Testing of a General Amber Force Field. Journal of Computational Chemistry 2004, 25, 1157-1174.

[35] Dick, B. G.; Overhauser, A. W. Theory of the Dielectric Constants of Alkali Halide Crystals. Physical Review 1958, 112, 90-103. 
[36] Ji, J.-Y.; Wu, S. Y. Proteresis of Core-Shell Nanocrystals: Investigation through Theoretical Simulation and Experimental Analysis. In Magnetic Materials; Maaz, K., Ed.; InTech: Rijeka, 2016; Chapter 6.

[37] Lim, T.-C. Modification of Morse Potential in Conventional Force Fields for Applying FPDP Parameters. Journal of Mathematical Chemistry 2010, 47, 984-989.

[38] Morse, P. M. Diatomic Molecules According to the Wave Mechanics. II. Vibrational Levels. Physical Review 1929, 34, 57-64.

[39] Guo, W.; Wang, D.; Hu, J.; Tang, Z. K.; Du, S. Raman Spectroscopy of Iodine Molecules Trapped in Zeolite Crystals. Applied Physics Letters 2011, 98, 043105 .

[40] Axilrod, B. M.; Teller, E. Interaction of the van der Waals Type Between Three Atoms. Journal of Chemical Physics 1943, 11, 299-300.

[41] Sadus, R. J. Exact Calculation of the Effect of Three-body Axilrod-Teller Interactions on Vapour-Liquid Phase Coexistence. Fluid Phase Equilibria 1998, $144,351-360$.

[42] Attard, P. Simulation Results for A Fluid With the Axilrod-Teller Triple Dipole Potential. Physical Review A 1992, 45, 5649-5653.

[43] Gwai, C. D. L. Structures and Properties of Liquid Crystals and Related Molecules from Computer Simulation. PhD dissertation, Durham University, 2002 .

[44] Hill, J.-R.; Sauer, J. Molecular Mechanics Potential for Silica and Zeolite Catalysts Based on ab Initio Calculations. 2. Aluminosilicates. Journal of Physical Chemistry 1995, 99, 9536-9550.

[45] Kermode, J. R.; Cereda, S.; Tangney, P.; Vita, A. D. A First Principles Based Polarizable $O(N)$ Interatomic Force Field for Bulk Silica. Journal of Chemical Physics 2010, 133, 094102.

[46] Rappé, A. K.; Goddard, W. A., III Charge Equilibration for Molecular Dynamics Simulations. Journal of Physical Chemistry 1991, 95, 3358-3363.

[47] Rick, S. W.; Stuart, S. J.; Berne, B. J. Dynamical Fluctuating Charge Force Fields: Application to Liquid Water. Journal of Chemical Physics 2003, 6, 3025-3039.

[48] Lemkul, J. A.; Huang, J.; Roux, B.; MacKerell, A. D., Jr. An Empirical Polarizable Force Field Based on the Classical Drude Oscillator Model: Development History and Recent Applications. Chemical Reviews 2016, 116, 4983-5013. 
[49] Sprik, M.; Klein, M. L. A Polarizable Model for Water Using Distributed Charge Sites. Journal of Chemical Physics 1988, 89, 7556-7560.

[50] Patel, S.; Brooks, C. L., III CHARMM Fluctuating Charge Force Field for Proteins: I Parameterization and Application to Bulk Organic Liquid Simulations. Journal of Computional Chemistry 2004, 25, 1-15.

[51] Tangney, P.; Scandolo, S. An ab initio Parametrized Interatomic Force Field for Silica. Journal of Chemical Physics 2002, 117, 8898-8904.

[52] Yamamoto, M. Charge-charge, Charge-dipole, Dipole-charge, Dipoledipole Interaction. https://janheyda.files.wordpress.com/2015/08/ electrostatics-multipoles.pdf, 2008.

[53] Berendsen, H. J. C.; Grigera, J. R.; Straatsma, T. P. The Missing Term in Effective Pair Potentials. Journal of Physical Chemistry 1987, 91, 6269-6271.

[54] Streitz, F. H.; Mintmire, J. W. Electrostatic Potentials for Metal-oxide Surfaces and Interfaces. Physical Review B 1994, 50, 11996-12003.

[55] Lamoureux, G.; Roux, B. Modeling Induced Polarization with Classical Drude Oscillators: Theory and Molecular Dynamics Simulation Algorithm. Journal of Physical Chemistry 1994, 101, 6141-6156.

[56] Rick, S. W.; Stuart, S. J. Potentials and Algorithms for Incorporating Polarizability in Computer Simulations. Reviews in Computational Chemistry 2002, 18, 89-146.

[57] Lamoureux, G.; MacKerell, A. D., Jr.; Roux, B. A Simple Polarizable Model of Water Based on Classical Drude Oscillators. Journal of Chemical Physics 2003, 119, 5185-5197.

[58] Jiang, W.; Hardy, D. J.; Phillips, J. C.; MacKerell, A. D., Jr.; Schulten, K.; Roux, B. High-Performance Scalable Molecular Dynamics Simulations of a Polarizable Force Field Based on Classical Drude Oscillators in NAMD. Journal of Physical Chemistry Letters 2011, 2, 87-92.

[59] Chowdhary, J.; Harder, E.; Lopes, P. E. M.; Huang, L.; MacKerell, A. D., Jr.; Roux, B. A Polarizable Force Field of Dipalmitoylphosphatidylcholine Based on the Classical Drude Model for Molecular Dynamics Simulations of Lipids. Journal of Physical Chemistry B 2013, 117, 9142-9160.

[60] Mitchell, P. J.; Fincham, D. Shell Model Simulations by Adiabatic Dynamics. Journal of Physics: Condensed Matter 1993, 5, 1031.

[61] Schröder, K.-P.; Sauer, J. Potential Functions for Silica and Zeolite Catalysts Based on ab Initio Calculations. 3. A Shell Model Ion Pair Potential for Silica and Aluminosilicates. Journal of Physical Chemistry 1996, 100, 11043-11049. 
[62] Kendall, K.; Roberts, A. D. Van der Waals Forces Influencing Adhesion of Cells. Philosophical Transactions B 2014, 370.

[63] Lu, Z.; Dunn, M. L. Van der Waals Adhesion of Graphene Membranes. Journal of Applied Physics 2010, 10\%, 044301.

[64] Tao, J.; Rappe, A. M. Physical Adsorption: Theory of van der Waals Interactions between Particles and Clean Surfaces. Physical Review Letters 2014, 112, 106101-106106.

[65] Leite, F. L.; Bueno, C. C.; Róz, A. L. D.; Ziemath, E. C.; Oliveira, O. N., Jr. Theoretical Models for Surface Forces and Adhesion and Their Measurement Using Atomic Force Microscopy. International Journal of Molecular Science 2012, 13, 12773-12856.

[66] London, F. The General Theory of Molecular Forces. Transactions of the Faraday Society 1937, 33, 8-26.

[67] Jeziorski, B.; Moszynski, R.; Szalewicz, K. Perturbation Theory Approach to Intermolecular Potential Energy Surfaces of van der Waals Complexes. Chemical Reviews 1994, 94, 1887-1930.

[68] Lopes, P. E. M.; Guvench, O.; MacKerell, A. D., Jr. Current Status of Protein Force Fields for Molecular Dynamics Simulations. In Molecular Modeling of Proteins; Springer New York: New York, NY, 2015; pp 47-71.

[69] MacKerell, A. D., Jr. Empirical Force Fields for Biological Macromolecules: Overview and Issues. Journal of Computational Chemistry. 2004, 25, 15841604.

[70] Kramer, G. J.; Farragher, N. P.; van Beest, B. W. H.; van Santen, R. A. Interatomic Force Fields for Silicas, Aluminophosphates, and Zeolites: Derivation Based on ab initio Calculations. Physical Review B 1991, 43, 5068-5080.

[71] Hopfinger, A. J.; Pearlstein, R. A. Molecular Mechanics Force-field Parameterization Procedures. Journal of Computational Chemistry 1984, 5, 486-499.

[72] Chirlian, L. E.; Francl, M. M. Atomic Charges Derived from Electrostatic Potentials: A Detailed Study. Journal of Computational Chemistry 1987, 8, 894-905.

[73] Henchman, R. H.; Essex, J. W. Generation of OPLS-like Charges from Molecular Electrostatic Potential Using Restraints. Journal of Computational Chemistry 1999, 20, 483-498.

[74] Cheung, D. L.; Clark, S. J.; Wilson, M. R. Parametrization and Validation of A Force Field for Liquid-Crystal Forming Molecules. Physical Review E 2002, 65, 051709-051720. 
[75] Wilson, E. B.; Decius, J. C.; Cross, P. C.; Sundheim, B. R. Molecular Vibrations: The Theory of Infrared and Raman Vibrational Spectra. Journal of the Electrochemical Society 1955, 102, 235C-236C.

[76] Califano, S. Vibrational States; Wiley: New York, 1976.

[77] Mott, A. J. Calculating Infrared Spectra of Proteins and Other Organic Molecules Based on Normal Modes. PhD dissertation, Arizona State University, 2012.

[78] Neugebauer, J.; Reiher, M.; Kind, C.; Hess, B. A. Quantum Chemical Calculation of Vibrational Spectra of Large Molecules-Raman and IR Spectra for Buckminsterfullerene. Journal of Computational Chemistry 2002, 23, 895-910.

[79] Alder, B. J.; Wainwright, T. E. Studies in Molecular Dynamics. I. General Method. Journal of Chemical Physics 1959, 31, 459-466.

[80] Verlet, L. Computer "Experiments" on Classical Fluids. I. Thermodynamical Properties of Lennard-Jones Molecules. Physical Review 1967, 159, 98-103.

[81] Swope, W. C.; Andersen, H. C.; Berens, P. H.; Wilson, K. R. A Computer Simulation Method for the Calculation of Equilibrium Constants for the Formation of Physical Clusters of Molecules: Application to Small Water Clusters. Journal of Chemical Physics 1982, 76, 637-649.

[82] Gunsteren, W. F. V.; Berendsen, H. J. C. A Leap-frog Algorithm for Stochastic Dynamics. Molecular Simulation 1988, 1, 173-185.

[83] Hünenberger, P. H. Thermostat Algorithms for Molecular Dynamics Simulations. In Advanced Computer Simulation: Approaches for Soft Matter Sciences I; Christian, H., Kurt, K., Eds.; Advances in Polymer Science; Springer: Berlin, 2005; pp 105-149.

[84] Nosé, S. A Unified Formulation of the Constant Temperature Molecular Dynamics Methods. Journal of Chemical Physics 1984, 81, 511-519.

[85] Hoover, W. G. Canonical Dynamics: Equilibrium Phase-space Distributions. Physical Review A 1985, 31, 1695-1697.

[86] Frenkel, D.; Smit, B. Appendix C - Linear Response Theory. In Understanding Molecular Simulation; Academic Press: San Diego, 2002; pp 509-523.

[87] Brehm, M.; Kirchner, B. TRAVIS - A Free Analyzer and Visualizer for Monte Carlo and Molecular Dynamics Trajectories. Journal of Chemical Information and Modeling 2011, 51, 2007-2023.

[88] Thomas, M.; Brehm, M.; Kirchner, B. Voronoi Dipole Moments for the Simulation of Bulk Phase Vibrational Spectra. Physical Chemistry Chemical Physics 2015, 17, 3207-3213. 
[89] Van De Graaf, B.; Njo, S. L.; Smirnov, K. S. Introduction to Zeolite Modeling. In Reviews in Computational Chemistry; John Wiley \& Sons, Inc.: Hoboken, NJ, 2007; Vol. 14; pp 137-223.

[90] Smith, J. O. Mathematics of the Discrete Fourier Transform (DFT); BookSurge Publishing: North Charleston SC, 2007.

[91] Richardson, J. W.; Pluth, J. J.; Smith, J. V.; Dytrych, W. J.; Bibby, D. M. Conformation of Ethylene Glycol and Phase Change in Silica Sodalite. Journal of Physical Chemistry 1988, 92, 243-247.

[92] Hazen, R. M.; Sharp, Z. D. Compressibility of sodalite and scapolite. American Mineralogist 1988, 73, 1120-1122.

[93] Kihara, K. An X-ray Study of the Temperature Dependence of the Quartz Structure. European Journal of Mineralogy 1990, 2, 63-78.

[94] Levien, L.; Prewitt, C. T.; Weidner, D. J. Structure and Elastic Properties of Quartz at Pressure. American Mineralogist 1980, 65, 920-930.

[95] Hriljac, J.; Eddy, M.; Cheetham, A.; Donohue, J.; Ray, G. Powder Neutron Diffraction and ${ }^{29} \mathrm{Si}$ MAS NMR Studies of Siliceous Zeolite-Y. Journal of Solid State Chemistry 1993, 106, 66-72.

[96] Martin, P. A. Strong Negative Thermal Expansion in Siliceous Faujasite. Chemical Communications 1998, 601-602.

[97] Colligan, M.; Forster, P. M.; Cheetham, A. K.; Lee, Y.; Vogt, T.; Hriljac, J. A. Synchrotron X-ray Powder Diffraction and Computational Investigation of Purely Siliceous Zeolite Y under Pressure. Journal of the American Chemical Society 2004, 126, 12015-12022.

[98] Downs, R. T.; Palmer, D. C. The Pressure Behavior of $\alpha$ Cristobalite. American Mineralogist 1994, 79, 9-14.

[99] Bhange, D.; Ramaswamy, V. Negative Thermal Expansion in Silicalite-1 and Zirconium silicalite-1 Having MFI Structure. Materials Research Bulletin 2006, 41, 1392-1402.

[100] van Koningsveld, H.; Jansen, J.; van Bekkum, H. The Monoclinic Framework Structure of Zeolite H-ZSM-5. Comparison with the Orthorhombic Framework of As-synthesized ZSM-5. Zeolites 1990, 10, 235-242.

[101] Sanchez-Valle, C.; Lethbridge, Z. A. D.; Sinogeikin, S. V.; Williams, J. J.; Walton, R. I.; Evans, K. E.; Bass, J. D. Negative Poisson's Ratios in Siliceous Zeolite MFI-silicalite. Journal of Chemical Physics 2008, 128, 184503. 
[102] Flanigen, E. M.; Khatami, H.; Szymanski, H. A. Infrared Structural Studies of Zeolite Frameworks. In Molecular Sieve Zeolites-I; Flanigen, E. M., Sand, L. B., Eds.; Advances in Chemistry; American Chemical Society: Washington, 1974; Vol. 101; Chapter 16, pp 201-229.

[103] Bueno-Pérez, R.; Calero, S.; Dubbeldam, D.; Ania, C. O.; Parra, J. B.; Zaderenko, A. P.; Merkling, P. J. Zeolite Force Fields and Experimental Siliceous Frameworks in a Comparative Infrared Study. Journal of Physical Chemistry C 2012, 116, 25797-25805.

[104] Jacobs, W. P. J. H.; van Wolput, J. H. M. C.; van Santen, R. A. Fouriertransform Infrared Study of the Protonation of the Zeolitic Lattice. Influence of Silicon : Aluminium Ratio and Structure. Journal of the Chemical Society, Faraday Transactions. 1993, 89, 1271-1276.

[105] Mozgawa, W.; Jastrzebski, W.; Handke, M. Vibrational Spectra of D4R and D6R Structural Units. Journal of Molecular Structure 2005, 744-747, 663-670.

[106] Frisch, M. J.; Trucks, G. W.; Schlegel, H. B.; Scuseria, G. E.; Robb, M. A.; Cheeseman, J. R.; Scalmani, G.; Barone, V.; Petersson, G. A.; Nakatsuji, H.; Li, X.; Caricato, M.; Marenich, A. V.; Bloino, J.; Janesko, B. G.; Gomperts, R.; Mennucci, B.; Hratchian, H. P.; Ortiz, J. V.; Izmaylov, A. F.; Sonnenberg, J. L.; Williams-Young, D.; Ding, F.; Lipparini, F.; Egidi, F.; Goings, J.; Peng, B.; Petrone, A.; Henderson, T.; Ranasinghe, D.; Zakrzewski, V. G.; Gao, J.; Rega, N.; Zheng, G.; Liang, W.; Hada, M.; Ehara, M.; Toyota, K.; Fukuda, R.; Hasegawa, J.; Ishida, M.; Nakajima, T.; Honda, Y.; Kitao, O.; Nakai, H.; Vreven, T.; Throssell, K.; Montgomery, J. A., Jr.; Peralta, J. E.; Ogliaro, F.; Bearpark, M. J.; Heyd, J. J.; Brothers, E. N.; Kudin, K. N.; Staroverov, V. N.; Keith, T. A.; Kobayashi, R.; Normand, J.; Raghavachari, K.; Rendell, A. P.; Burant, J. C.; Iyengar, S. S.; Tomasi, J.; Cossi, M.; Millam, J. M.; Klene, M.; Adamo, C.; Cammi, R.; Ochterski, J. W.; Martin, R. L.; Morokuma, K.; Farkas, O.; Foresman, J. B.; Fox, D. J. Gaussian 09, Revision D.01. 2016; Gaussian Inc. Wallingford CT.

[107] Scott, A. P.; Radom, L. Harmonic Vibrational Frequencies: An Evaluation of Hartree-Fock, Møller-Plesset, Quadratic Configuration Interaction, Density Functional Theory, and Semiempirical Scale Factors. Journal of Physical Chemistry 1996, 100, 16502-16513.

[108] Dennington, R.; Keith, T.; Millam, J. GaussView Version 5. Semichem Inc., Shawnee Mission, KS, 2009.

[109] Bornhauser, P.; Bougeard, D. Intensities of the Vibrational Spectra of Siliceous Zeolites by Molecular Dynamics Calculations. I. Infrared Spectra. Journal of Physical Chemistry B 2001, 105, 36-41. 
[110] Liang, Y.; Miranda, C. R.; Scandolo, S. Infrared and Raman Spectra of Silica Polymorphs from an ab initio Parametrized Polarizable Force Field. Journal of Chemical Physics 2006, 125, 194524.

[111] Serrano, D. P.; Li, H.-X.; Davis, M. E. Synthesis of Titanium-containing ZSM48. Journal of Chemical Society, Chemical Communications. 1992, 745-747.

[112] Bondi, A. Van der Waals Volumes and Radii. Journal of Physical Chemistry 1964, 68, 441-451.

[113] Rappé, A. K.; Casewit, C. J.; Colwell, K. S.; Goddard, W. A., III; Skiff, W. M. UFF, a Full Periodic Table Force Field for Molecular Mechanics and Molecular Dynamics Simulations. Journal of the American Chemical Society 1992, 114, 10024-10035.

[114] Seminario, J. M. Calculation of Intramolecular Force Fields from Secondderivative Tensors. International Journal of Quantum Chemistry 1996, 60, 1271-1277.

[115] Reyes, L. H.; Seminario, J. M. Determination of Precise Harmonic Force Constants for Alanine Polypeptides. Journal of Molecular Structure: THEOCHEM 2007, 818, 125-129.

[116] Hammond, K. D. Quantifying Defects in Zeolites and Zeolite Membranes. PhD dissertation, University of Massachusetts Amherst, 2010.

[117] Siebert, F.; Hildebrandt, P. Theory of Infrared Absorption and Raman Spectroscopy. In Vibrational Spectroscopy in Life Science; Wiley-VCH Verlag GmbH \& Co. KGaA, 2008; pp 11-61.

[118] Stackhouse, S.; Stixrude, L. Theoretical Methods for Calculating the Lattice Thermal Conductivity of Minerals. Reviews in Mineralogy and Geochemistry 2010, 71, 253-269.

[119] French, R. H.; Parsegian, V. A.; Podgornik, R.; Rajter, R. F.; Jagota, A.; Luo, J.; Asthagiri, D.; Chaudhury, M. K.; Chiang, Y.-m.; Granick, S.; Kalinin, S.; Kardar, M.; Kjellander, R.; Langreth, D. C.; Lewis, J.; Lustig, S.; Wesolowski, D.; Wettlaufer, J. S.; Ching, W.-Y.; Finnis, M.; Houlihan, F.; von Lilienfeld, O. A.; van Oss, C. J.; Zemb, T. Long Range Interactions in Nanoscale Science. Review of Modern Physics 2010, 82, 1887-1944.

[120] Jorgensen, W. L.; Tirado-Rives, J. The OPLS [Optimized Potentials for Liquid Simulations] Potential Functions for Proteins, Energy Minimizations for Crystals of Cyclic Peptides and Crambin. Journal of the American Chemical Society 1988, 110, 1657-1666.

[121] Minachev, K. M.; Isakov, Y. I. Catalytic Properties of ZeolitesA General Review. In Molecular Sieves; 1973; Chapter 40, pp 451-460. 
[122] Rhodes, C. J. Properties and Applications of Zeolites. Science Progress 2010, 93, 223-284.

[123] Bornhauser, P.; Calzaferri, G. Ring-Opening Vibrations of Spherosiloxanes. Journal of Physical Chemistry 1996, 100, 2035-2044.

[124] Mortier, W. J.; Ghosh, S. K.; Shankar, S. Electronegativity-equalization Method for the Calculation of Atomic Charges in Molecules. Journal of the American Chemical Society 1986, 108, 4315-4320.

[125] Cinar, S.; Beler-Baykal, B. Ion Exchange with Natural Zeolites: An Alternative for Water Softening. Water Science and Technology 2005, 51, 71-77. 